APÉNDICE: TABLAS COMPARATIVAS 


\section{INDICE DE TABLAS}

TABLA 1: Se redujo la capacidad de sembradura y de producción de los préstamos adosados a cada uno de los solares a una única medida de capacidad: HEMINAS DE TRIGO de sembradura para las tierras de cereal, AZUMBRES DE VINO para las viñas, CARGAS DE YERBA para los prados y HEMINAS DE LINO para los huertos, con el objeto de visualizar de manera más clara las diferentes dimensiones de las unidades de explotación campesina. La misma operación se realizó con respecto a la renta debida por los solares, aspecto que permite observar la relación entre capacidad de producción y monto de la infurción. En los casos en que el tributo exigido por el señor incorpora productos que no se derivan de la producción de los préstamos cedidos se indicó con color (azul para el caso de cereales y rosa cuando se trata de vino). .p. 226

TABLA 2: Se realizó una tabla comparativa a partir de los datos ofrecidos por los tres apeos conservados: el de 1499, 1515 y 1538. En este caso se llevó a cabo un seguimiento de los mismos solares a través del tiempo (consignándose los titulares, los bienes adosados en forma de préstamos y la renta exigida por el señor) pudiendo detectar las variaciones o su inmutabilidad en los cuarenta años que separa la redacción de los apeos. Hemos seleccionado como representativo del centro del dominio al concejo de Santibañez y de la periferia a las aldeas ubicadas en San Sebastián y en Valdeprado

..p. 238

\begin{tabular}{|c|c|c|c|c|c|c|c|c|c|c|c|c|}
\hline TABLA & 3: & Se & realizó & la & misma & tarea & con & los & tercios & de & Santibañez, & Potes $\quad \mathrm{y}$ \\
\hline
\end{tabular}

TABLA 4: Se refleja el seguimiento de las heredades cedidas en arrendamiento al concejo de Colio.. 
TABLA 5: Se indica en cada una de las aldeas lebaniegas donde el monasterio posee solares y préstamos la cantidad de unidades de explotación simple (compuestas solamente por tierras de cereal, viñas, prados o huertos) y el número de unidades de explotación complejas teniendo en cuenta su composición. El total relativo a cada uno de los lugares fue expresado en números y

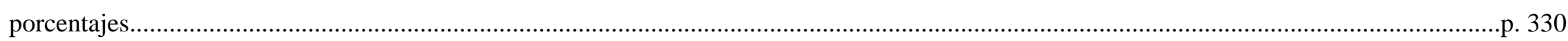


SOLARES Y PRÉSTAMOS -APEO DE 1499-

\begin{tabular}{|c|c|c|c|c|c|c|}
\hline LUGAR & SOLAR & $\begin{array}{l}\text { TIERRAS } \\
\text { (sembradura) }\end{array}$ & VIÑAS & PRADOS & HUERTOS & RENTA \\
\hline \multirow[t]{12}{*}{ ARGÜÉBANES } & Solar de la Escuentra & 2 HEMINAS & - & 12 CARGAS & - & 4 HEMINAS DE TRIGO \\
\hline & Solar de Aldonza & 2 HEMINAS & $\begin{array}{c}192 \\
\text { AZUMBRES }\end{array}$ & - & - & $\begin{array}{l}6 \text { HEMINAS DE TRIGO Y } \\
1 \text { GALLINA }\end{array}$ \\
\hline & Otro solar & 2 HEMINAS & 96 AZUMBRES & - & - & 8 HEMINAS DE TRIGO \\
\hline & Solar de la Rebeca & 6 HEMINAS & - & $\dot{¿} ?$ & $\dot{¿} ?$ & $\begin{array}{l}6 \text { HEMINAS DE TRIGO Y } \\
8 \text { AZUMBRES DE VINO }\end{array}$ \\
\hline & Solar del Behenal & $21 / 2$ HEMINAS & - & - & - & 4 HEMINAS DE TRIGO \\
\hline & Solar derrocado & 3 HEMINAS & 1 CARGA & - & - & 3 HEMINAS DE TRIGO \\
\hline & $\begin{array}{l}\text { Solar de García de la } \\
\text { Ermida }\end{array}$ & 6 HEMINAS & - & 8 CARGAS & - & 5 HEMINAS DE TRIGO \\
\hline & $\begin{array}{l}\text { En la entrada del } \\
\text { Behenal }\end{array}$ & 7 HEMINAS & - & 12 CARGAS & - & 5 HEMINAS DE TRIGO \\
\hline & En el Arrial & $41 / 2$ HEMINAS & 64 AZUMBRES & - & - & 10 HEMINAS DE TRIGO \\
\hline & En el Arrial & 5 HEMINAS & - & 2 CARGAS & - & 10 HEMINAS DE TRIGO \\
\hline & Solar del Llano & 16 HEMINAS & - & - & - & 15 HEMINAS DE TRIGO \\
\hline & Solar en Cotillo & 8 HEMINAS & - & - & - & 3 HEMINAS DE TRIGO \\
\hline \multirow[t]{7}{*}{ CONGARNA } & & 66 HEMINAS & - & 60 CARGAS & - & 16 HEMINAS DE TRIGO \\
\hline & Otro solar & $\begin{array}{c}101 / 2 \\
\text { HEMINAS }\end{array}$ & 32 AZUMBRES & 3 CARGAS & - & 4 HEMINAS DE TRIGO \\
\hline & $\begin{array}{l}\text { Solar de Juan de } \\
\text { Diego }\end{array}$ & 20 HEMINAS & $\begin{array}{c}416 \\
\text { AZUMBRES }\end{array}$ & $1 \mathrm{COLONO}$ & - & $\begin{array}{c}16 \text { HEMINAS DE TRIGO, } \\
32 \text { AZUMBRES DE VINO } \\
\text { Y } 3 \text { HUEBRAS }\end{array}$ \\
\hline & $\begin{array}{l}\text { Solar de hijos de } \\
\text { Pedro Martínez }\end{array}$ & 4 HEMINAS & $\begin{array}{c}160 \\
\text { AZUMBRES } \\
\end{array}$ & - & 1 ERA & 14 AZUMBRES DE VINO \\
\hline & $\begin{array}{l}\text { Solar de Toribio } \\
\text { Barrial }\end{array}$ & 8 1/2 HEMINAS & 96 AZUMBRES & - & $\dot{d} ?$ & 4 HEMINAS DE TRIGO \\
\hline & $\begin{array}{l}\text { Solar de Toribio el } \\
\text { Almirante }\end{array}$ & 5 1/2 HEMINAS & $\begin{array}{c}136 \\
\text { AZUMBRES } \\
\end{array}$ & - & - & 4 HEMINAS DE TRIGO \\
\hline & Solar del Oso & 1 HEMINA & $\begin{array}{c}240 \\
\text { AZUMBRES }\end{array}$ & - & - & $\begin{array}{l}5 \text { HEMINAS DE TRIGO Y } \\
7 \text { AZUMBRES DE VINO }\end{array}$ \\
\hline
\end{tabular}




\begin{tabular}{|c|c|c|c|c|c|c|}
\hline & $\begin{array}{l}\text { Solar de Toribio de } \\
\text { Juana }\end{array}$ & 12 HEMINAS & - & 4 CARGAS & - & 6 HEMINAS DE TRIGO \\
\hline & $\begin{array}{l}\text { Solar de la Canal } \\
\text { (la parte de Diego de } \\
\text { Baró) }\end{array}$ & $1 \frac{1}{2} 2$ HEMINA & $\begin{array}{c}104 \\
\text { AZUMBRES }\end{array}$ & 2 CARGAS & - & $\begin{array}{c}2 \text { HEMINAS DE TRIGO Y } \\
4 \text { AZUMBRES DE VINO } \\
2 \text { HEMINAS DE TRIGO, } 4 \\
\text { AZUMBRES DE VINO Y } \\
\text { 1 GALLINA }\end{array}$ \\
\hline \multirow[t]{3}{*}{ FLORANES } & & 10 HEMINAS & $\begin{array}{c}528 \\
\text { AZUMBRES }\end{array}$ & - & $\begin{array}{c}1 \text { CASAR } \\
1 \text { CAMPO c/ } \\
\text { ÁRBOLES }\end{array}$ & $\begin{array}{c}14 \text { HEMINAS DE TRIGO, } \\
28 \text { AZUMBRES DE VINO, } \\
3 \text { HUEBRAS Y } 1 \\
\text { GALLINA }\end{array}$ \\
\hline & $\begin{array}{l}\text { Solar de Ferrando de } \\
\text { Baró }\end{array}$ & - & $\begin{array}{c}272 \\
\text { AZUMBRES }\end{array}$ & - & - & $\begin{array}{c}8 \text { HEMINAS DE TRIGO, } 4 \\
\text { AZUMBRES DE VINO Y } \\
1 \text { HUEBRA }\end{array}$ \\
\hline & $\begin{array}{l}\text { Solar de Gonzalo de } \\
\text { Floranes } \\
\text { Solar de Juan de } \\
\text { Treceño }\end{array}$ & - & $\begin{array}{c}168 \\
\text { AZUMBRES } \\
\\
920 \\
\text { AZUMBRES }\end{array}$ & - & - & $\begin{array}{c}18 \text { HEMINAS DE TRIGO } \\
\text { Y } 4 \text { AZUMBRES DE } \\
\text { VINO }\end{array}$ \\
\hline \multirow[t]{5}{*}{ TURIENO } & En el barrio de Era & $\begin{array}{c}2 / 3 \text { DE } \\
\text { HEMINA }\end{array}$ & 72 AZUMBRES & - & $\begin{array}{c}5 \text { 1/2 HEMINAS } \\
\text { DE LINO }\end{array}$ & $\begin{array}{c}2 \text { HEMINAS DE TRIGO, } 4 \\
\text { AZUMBRES DE VINO Y } \\
1 \text { HUEBRA }\end{array}$ \\
\hline & Solar de Era & 6 HEMINAS & $\begin{array}{c}624 \\
\text { AZUMBRES }\end{array}$ & - & - & $\begin{array}{c}10 \text { HEMINAS DE TRIGO, } \\
14 \text { AZUMBRES DE VINO } \\
\text { Y } 3 \text { HUEBRAS }\end{array}$ \\
\hline & $\begin{array}{l}\text { Solar de Gonzalo } \\
\text { Sánchez }\end{array}$ & 7 HEMINAS & 72 AZUMBRES & 1 CARGA & - & $\begin{array}{l}2 \text { HEMINAS DE TRIGO Y } \\
4 \text { AZUMBRES DE VINO }\end{array}$ \\
\hline & $\begin{array}{l}\text { Solar de Domingo } \\
\text { Fernández }\end{array}$ & 2 HEMINAS & $\begin{array}{c}304 \\
\text { AZUMBRES }\end{array}$ & - & $\begin{array}{l}4 \text { HEMINAS } \\
\text { DE LINO }\end{array}$ & $\begin{array}{l}7 \text { HEMINAS DE TRIGO Y } \\
16 \text { AZUMBRES DE VINO }\end{array}$ \\
\hline & Solar de García & - & $\begin{array}{c}824 \\
\text { AZUMBRES }\end{array}$ & - & $\begin{array}{l}\text { 2/3 DE } \\
\text { HEMINA DE } \\
\text { LINO }\end{array}$ & $\begin{array}{c}10 \text { HEMINAS DE TRIGO, } \\
14 \text { AZUMBRES DE VINO } \\
\text { Y } 3 \text { HUEBRAS }\end{array}$ \\
\hline
\end{tabular}




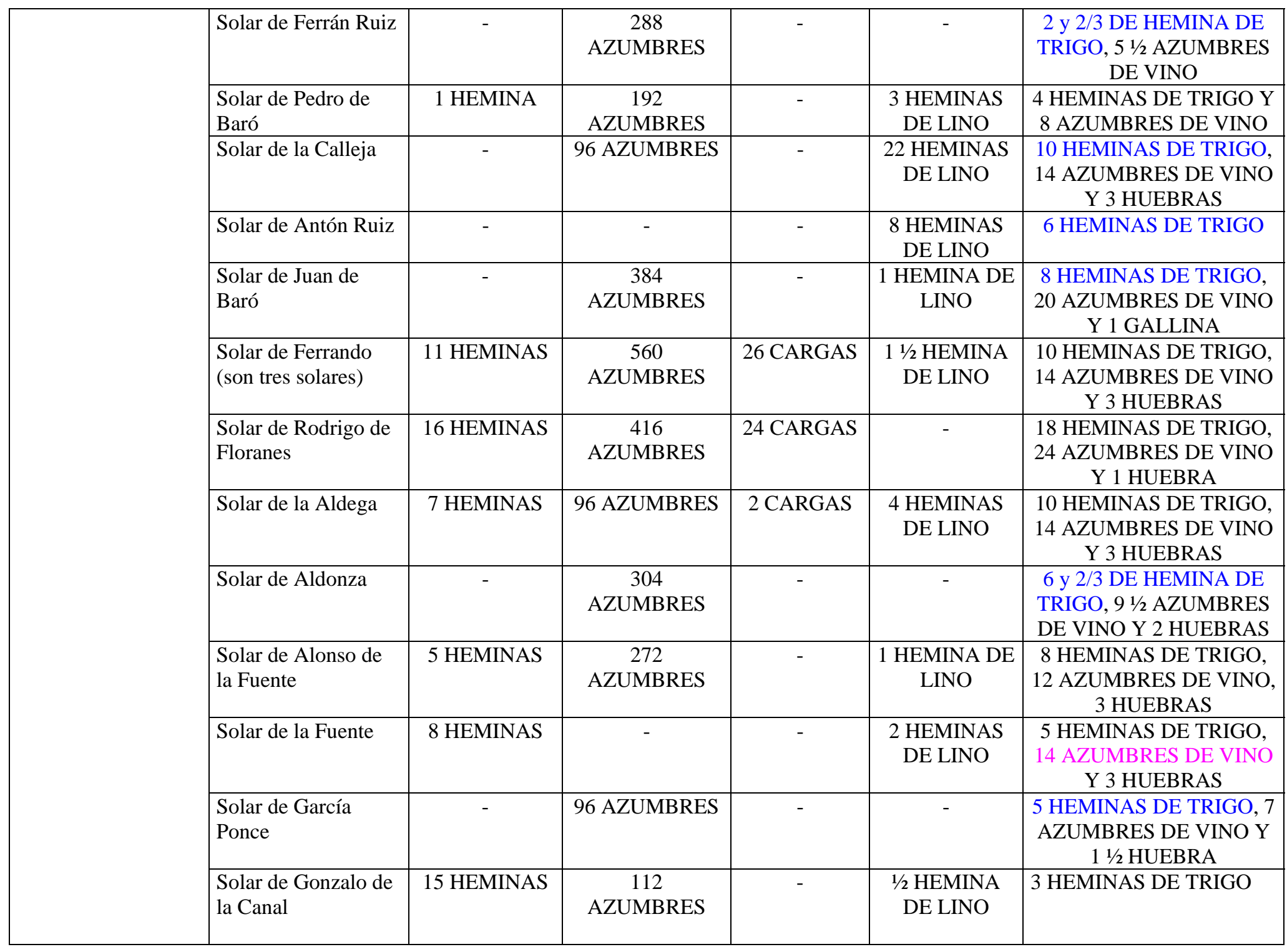




\begin{tabular}{|c|c|c|c|c|c|c|}
\hline & $\begin{array}{l}\text { Solar de Gonzalo de } \\
\text { la Calleja } \\
\text { (en la Calleja) }\end{array}$ & 2 HEMINAS & 48 AZUMBRES & $\begin{array}{l}\text { 3 CARGAS } \\
3 \\
\text { MOSTELAS } \\
\end{array}$ & - & 4 HEMINAS DE TRIGO \\
\hline & $\begin{array}{l}\text { Solar de Gonzalo } \\
\text { Vallejo } \\
\text { (en la Calleja) }\end{array}$ & - & $\begin{array}{c}192 \\
\text { AZUMBRES }\end{array}$ & - & $\begin{array}{l}2 \text { HEMINAS } \\
\text { DE LINO }\end{array}$ & 8 HEMINAS DE TRIGO \\
\hline & Otro solar & - & - & - & $\begin{array}{l}1 / 2 \text { HEMINA } \\
\text { DE LINO }\end{array}$ & 1 HEMINA DE TRIGO \\
\hline \multirow[t]{5}{*}{ OTERO } & $\begin{array}{l}\text { Solar de Diego } \\
\text { Martínez }\end{array}$ & - & $\begin{array}{c}240 \\
\text { AZUMBRES }\end{array}$ & 12 CARGAS & $\begin{array}{l}2 \text { HEMINAS } \\
\text { DE LINO }\end{array}$ & $\begin{array}{l}3 \text { HEMINAS DE TRIGO Y } \\
4 \text { AZUMBRES DE VINO }\end{array}$ \\
\hline & $\begin{array}{l}\text { Solar de Pedro de } \\
\text { Otero }\end{array}$ & - & $\begin{array}{c}144 \\
\text { AZUMBRES }\end{array}$ & 2 CARGAS & $\dot{¿} ?$ & $\begin{array}{l}10 \text { HEMINAS DE TRIGO } \\
\text { Y } 10 \text { AZUMBRES DE } \\
\text { VINO }\end{array}$ \\
\hline & $\begin{array}{l}\text { Solar de Juan de } \\
\text { Armaño }\end{array}$ & 8 HEMINAS & $\begin{array}{c}264 \\
\text { AZUMBRES } \\
\end{array}$ & 18 CARGAS & - & 16 HEMINAS DE TRIGO \\
\hline & \begin{tabular}{|l} 
Solar del cura \\
\end{tabular} & - & $\begin{array}{c}400 \\
\text { AZUMBRES }\end{array}$ & - & - & $\begin{array}{l}10 \text { HEMINAS DE TRIGO } \\
\text { Y } 14 \text { AZUMBRES DE } \\
\text { VINO }\end{array}$ \\
\hline & Solar de Mesaina & 53 HEMINAS & $\begin{array}{c}432 \\
\text { AZUMBRES }\end{array}$ & 3 CARGAS & - & $\begin{array}{l}8 \text { HEMINAS DE TRIGO Y } \\
12 \text { AZUMBRES DE VINO }\end{array}$ \\
\hline \multirow[t]{6}{*}{ MIESES } & $\begin{array}{l}\text { En el barrio de } \\
\text { Armental }\end{array}$ & 2 HEMINAS & $\begin{array}{c}424 \\
\text { AZUMBRES }\end{array}$ & - & $\begin{array}{l}1 \text { CASAR } 1 \\
\text { CAMPO c/ } \\
\text { ARBOLES }\end{array}$ & $\begin{array}{l}10 \text { HEMINAS DE TRIGO, } \\
14 \text { AZUMBRES DE VINO, } \\
3 \text { HUEBRAS Y } 1 \\
\text { GALLINA }\end{array}$ \\
\hline & Solar de Catalina & 1 HEMINA & - & - & - & 3 PANES Y 1 GALLINA \\
\hline & $\begin{array}{l}\text { Solar de Ferrando de } \\
\text { Baró }\end{array}$ & 4 HEMINAS & $\begin{array}{c}176 \\
\text { AZUMBRES }\end{array}$ & - & $\begin{array}{l}2 \text { HEMINAS } \\
\text { DE LINO }\end{array}$ & $\begin{array}{l}7 \text { HEMINAS DE TRIGO Y } \\
14 \text { AZUMBRES DE VINO }\end{array}$ \\
\hline & $\begin{array}{l}\text { Solar de la } \\
\text { Corralona }\end{array}$ & 16 HEMINAS & $\begin{array}{c}224 \\
\text { AZUMBRES }\end{array}$ & - & $\begin{array}{l}3 \text { HEMINAS } \\
\text { DE LINO }\end{array}$ & $\begin{array}{l}12 \text { HEMINAS DE TRIGO, } \\
14 \text { AZUMBRES DEE } \\
\text { VINO Y } 1 \frac{1}{2} \text { HUEBRA }\end{array}$ \\
\hline & Solar de la Cortina & - & $\begin{array}{c}168 \\
\text { AZUMBRES }\end{array}$ & - & $\begin{array}{l}2 \text { HEMINAS } \\
\text { DE LINO }\end{array}$ & $\begin{array}{l}10 \text { HEMINAS DE TRIGO, } \\
14 \text { AZUMBRES DE VINO } \\
\text { Y } 3 \text { HUEBRAS }\end{array}$ \\
\hline & $\begin{array}{l}\text { Solar de Gonzalo de } \\
\text { Baró }\end{array}$ & 16 HEMINAS & - & - & - & 8 HEMINAS DE TRIGO \\
\hline
\end{tabular}




\begin{tabular}{|c|c|c|c|c|c|c|}
\hline & Solar de Caldevilla & 4 HEMINAS & $\begin{array}{c}192 \\
\text { AZUMBRES }\end{array}$ & 18 CARGAS & - & $\begin{array}{l}10 \text { HEMINAS DE TRIGO, } \\
14 \text { AZUMBRES DE VINO } \\
\text { Y } 3 \text { HUEBRAS }\end{array}$ \\
\hline & $\begin{array}{l}\text { Solar de Juan de } \\
\text { Mieses }\end{array}$ & 12 HEMINAS & $\begin{array}{c}168 \\
\text { AZUMBRES }\end{array}$ & - & $\begin{array}{l}1 \text { HEMINA DE } \\
\text { LINO }\end{array}$ & $\begin{array}{l}10 \text { HEMINAS DE TRIGO, } \\
14 \text { AZUMBRES DE VINO } \\
\text { Y } 3 \text { HUEBRAS }\end{array}$ \\
\hline & $\begin{array}{l}\text { Solar de Juan } \\
\text { Herrero }\end{array}$ & 4 HEMINAS & - & 1 CARGA & $\begin{array}{l}1 \text { HEMINA DE } \\
\text { LINO }\end{array}$ & $\begin{array}{l}10 \text { HEMINAS DE TRIGO } \\
\text { Y } 1 \text { GALLINA }\end{array}$ \\
\hline & Solar de Antón & 4 HEMINAS & $\begin{array}{c}264 \\
\text { AZUMBRES }\end{array}$ & & $\begin{array}{l}3 \text { HEMINAS } \\
\text { DE LINO }\end{array}$ & $\begin{array}{l}10 \text { HEMINAS DE TRIGO, } \\
14 \text { AZUMBRES DE VINO } \\
\text { Y } 3 \text { HUEBRAS }\end{array}$ \\
\hline & Solar de la Herrén & 17 HEMINAS & $\begin{array}{c}768 \\
\text { AZUMBRES }\end{array}$ & - & 4 NOGALES & $\begin{array}{l}15 \text { HEMINAS DE TRIGO, } \\
21 \text { AZUMBRES DE VINO } \\
\text { Y } 4 \text { 1 } 12 \text { HUEBRAS }\end{array}$ \\
\hline & Solar del curero & - & 48 AZUMBRES & 12 CARGAS & $\begin{array}{l}1 \text { HEMINA DE } \\
\text { LINO }\end{array}$ & $\begin{array}{l}5 \text { HEMINAS DE TRIGO Y } \\
7 \text { AZUMBRES DE VINO }\end{array}$ \\
\hline & $\begin{array}{l}\text { Solar de Hondón de } \\
\text { Villa }\end{array}$ & 4 HEMINAS & $\begin{array}{c}160 \\
\text { AZUMBRES }\end{array}$ & & & $\begin{array}{l}10 \text { HEMINAS DE TRIGO } \\
\text { Y } 14 \text { AZUMBRES DE } \\
\text { VINO }\end{array}$ \\
\hline & $\begin{array}{l}\text { Solar de Sancho de } \\
\text { Ruy Moroso }\end{array}$ & 2 HEMINAS & $\begin{array}{c}160 \\
\text { AZUMBRES }\end{array}$ & 4 CARGAS & $\begin{array}{l}4 \text { HEMINAS } \\
\text { DE LINO }\end{array}$ & $\begin{array}{l}16 \text { HEMINAS DE TRIGO } \\
\text { Y } 20 \text { AZUMBRES DE } \\
\text { VINO }\end{array}$ \\
\hline & $\begin{array}{l}\text { Solar de Alonso } \\
\text { González }\end{array}$ & 2 HEMINAS & $\begin{array}{c}120 \\
\text { AZUMBRES }\end{array}$ & - & $\begin{array}{l}1 / 2 \text { HEMINA } \\
\text { DE LINO }\end{array}$ & $\begin{array}{l}7 \text { HEMINAS DE TRIGO Y } \\
10 \text { AZUMBRES DE VINO }\end{array}$ \\
\hline BARÓ & & - & 64 AZUMBRES & - & - & $\begin{array}{l}3 \text { HEMINAS DE TRIGO Y } \\
3 \text { MARAVEDIES DEL } \\
\text { YANTAR }\end{array}$ \\
\hline & Otro solar & - & $\begin{array}{c}240 \\
\text { AZUMBRES }\end{array}$ & - & $\dot{¿} ?$ & $\begin{array}{l}4 \text { HEMINAS DE TRIGO Y } \\
4 \text { MARAVEDIES DEL } \\
\text { YANTAR }\end{array}$ \\
\hline & Solar en el Otero & 6 HEMINAS & - & - & - & $\begin{array}{l}2 \text { HEMINAS DE TRIGO Y } \\
2 \text { MARAVEDIES DEL } \\
\text { YANTAR }\end{array}$ \\
\hline & En el Otero & 1 1/2 HEMINA & 32 AZUMBRES & 2 CARGAS & - & $\begin{array}{l}3 \text { HEMINAS DE TRIGO Y } \\
3 \text { MARAVEDIES DEL } \\
\text { YANTAR }\end{array}$ \\
\hline
\end{tabular}




\begin{tabular}{|c|c|c|c|c|c|c|}
\hline & En el Otero & 3 HEMINAS & 80 AZUMBRES & - & - & $\begin{array}{l}3 \text { HEMINAS DE TRIGO Y } \\
3 \text { MARAVEDIES DEL } \\
\text { YANTAR }\end{array}$ \\
\hline & Otro solar & 14 HEMINAS & 32 AZUMBRES & - & $\dot{¿} ?$ & $\begin{array}{l}\text { 14 HEMINAS DE TRIGO } \\
\text { Y } 14 \text { MARAVEDIES DEL } \\
\text { YANTAR }\end{array}$ \\
\hline CAMALEÑO & Solar de Lama & 8 HEMINAS & - & - & - & $\begin{array}{l}5 \text { HEMINAS DE TRIGO Y } \\
5 \text { MARAVEDIES DEL } \\
\text { YANTAR }\end{array}$ \\
\hline \multirow[t]{3}{*}{ BODIA } & & 34 HEMINAS & - & 24 CARGAS & NOZALES & $\begin{array}{l}5 \text { HEMINAS DE TRIGO Y } \\
5 \text { MARAVEDIES DEL } \\
\text { YANTAR }\end{array}$ \\
\hline & Otro solar & - & - & 6 CARGAS & - & 3 PANES Y 1 GALLINA \\
\hline & Otro solar & - & - & 6 CARGAS & - & $\begin{array}{l}\text { LA } 1 / 2 \text { DE } 3 \text { PANES Y } 1 \\
\text { GALLINA }\end{array}$ \\
\hline \multirow[t]{4}{*}{ CARABAÑO } & & 4 HEMINAS & - & - & - & $\begin{array}{l}4 \text { HEMINAS DE TRIGO Y } \\
\text { 4 MARAVEDIES DEL } \\
\text { YANTAR }\end{array}$ \\
\hline & Otro solar & - & 64 AZUMBRES & - & - & $\begin{array}{l}4 \text { HEMINAS DE TRIGO Y } \\
4 \text { MARAVEDIES DEL } \\
\text { YANTAR }\end{array}$ \\
\hline & En el Hoyo & - & 64 AZUMBRES & - & - & $\begin{array}{l}3 \text { HEMINAS DE TRIGO, } 1 \\
\text { GALLINA Y } 3 \\
\text { MARAVEDIES DEL } \\
\text { YANTAR }\end{array}$ \\
\hline & $\begin{array}{l}\text { En el Hoyo de } \\
\text { Carabaño }\end{array}$ & - & 80 AZUMBRES & - & - & $\begin{array}{l}3 \text { HEMINAS DE TRIGO, } 1 \\
\text { GALLINA Y } 3 \\
\text { MARAVEDIES DEL } \\
\text { YANTAR }\end{array}$ \\
\hline \multirow[t]{2}{*}{ LA FRECHA } & & 9 HEMINAS & - & 36 CARGAS & - & $\begin{array}{l}9 \text { HEMINAS DE TRIGO, } 9 \\
\text { MARAVEDIES DEL } \\
\text { YANTAR Y } 2 \text { GALLINAS }\end{array}$ \\
\hline & Otro solar & 4 HEMINAS & $\begin{array}{c}336 \\
\text { AZUMBRES }\end{array}$ & 2 CARGAS & - & $\begin{array}{l}12 \text { HEMINAS DE TRIGO, } \\
12 \text { MARAVEDIES DEL } \\
\text { YANTAR Y } 2 \text { GALLINAS }\end{array}$ \\
\hline
\end{tabular}




\begin{tabular}{|c|c|c|c|c|c|c|}
\hline & Otro solar & - & 64 AZUMBRES & 4 CARGAS & $\dot{¿} ?$ & $\begin{array}{l}4 \text { HEMINAS DE TRIGO, } 4 \\
\text { MARAVEDIES DEL } \\
\text { YANTAR, } 3 \text { PANES Y } 1 \\
\text { GALLINA }\end{array}$ \\
\hline BEARES & & 2 HEMINAS & 20 AZUMBRES & - & - & $\begin{array}{l}3 \text { HEMINAS DE TRIGO Y } \\
3 \text { MARAVEDIES DEL } \\
\text { YANTAR }\end{array}$ \\
\hline \multirow[t]{13}{*}{ TANARRIO } & $\begin{array}{l}\text { Solar de cabo la } \\
\text { Fuente }\end{array}$ & 7 HEMINAS & 8 AZUMBRES & 36 CARGAS & - & $\begin{array}{l}5 \text { HEMINAS DE TRIGO, } 2 \\
\text { AZUMBRES, } 1 \\
\text { FACENDERA Y } \\
\text { YANTAR }\end{array}$ \\
\hline & Otro solar & $91 / 2$ HEMINAS & - & 18 CARGAS & ENCINAS & $\begin{array}{l}51 / 3 \text { HEMINAS DE } \\
\text { TRIGO, } 2 \text { AZUMBRES DE } \\
\text { VINO, } 1 \text { OBRERO, } \\
\text { YANTAR }\end{array}$ \\
\hline & Otro solar & 2 1/2 HEMINAS & 8 AZUMBRES & $\begin{array}{r}31 \text { CARGAS } \\
1 \text { COLONO } \\
\end{array}$ & - & $\begin{array}{l}4 \text { HEMINAS DE TRIGO, } 2 \\
\text { AZUMBRES DE VINO }\end{array}$ \\
\hline & Solar Errocieto & 3 HEMINAS & 24 AZUMBRES & 12 CARGAS & - & $\begin{array}{l}3 \text { 1/2 HEMINAS DE TRIGO } \\
\text { Y } 1 \text { AZUMBRE DE VINO }\end{array}$ \\
\hline & Solar de la Canal & - & - & 30 CARGAS & - & 2 1/2 HEMINAS DE TRIGO \\
\hline & Otro solar & $91 / 2$ HEMINAS & - & $\begin{array}{l}29 \text { CARGAS } \\
1 \text { COLONO }\end{array}$ & - & 12 HEMINAS DE TRIGO \\
\hline & $\begin{array}{l}\text { Solar de María } \\
\text { Izquierda }\end{array}$ & 1 HEMINA & - & - & - & 3 PANES Y 1 GALLINA \\
\hline & $\begin{array}{l}\text { En el barrio de } \\
\text { Encima }\end{array}$ & 6 HEMINAS & - & $\begin{array}{c}13^{1 / 2} \\
\text { CARGAS }\end{array}$ & ENCINAS & 2 1/2 HEMINAS DE TRIGO \\
\hline & En la era del Beato & $\begin{array}{c}71 / 2 \\
\text { HEMINAS }\end{array}$ & - & $\begin{array}{l}18 \text { CARGAS } \\
2 \text { COLONOS }\end{array}$ & $\begin{array}{c}\dot{2} ? \\
\text { ENCINAS }\end{array}$ & 5 1/4 HEMINAS DE TRIGO \\
\hline & Encima de la aldea & 9 HEMINAS & - & 18 CARGAS & ENCINAS & 2 1/2 HEMINAS DE TRIGO \\
\hline & En el Otero & $\begin{array}{c}3 \frac{1}{2} \\
\text { HEMINAS }\end{array}$ & 12 AZUMBRES & - & - & $\begin{array}{l}21 / 2 \text { HEMINAS DE TRIGO } \\
\text { Y } 2 \text { AZUMBRES DE } \\
\text { VINO }\end{array}$ \\
\hline & $\begin{array}{l}\text { Otro solar en el } \\
\text { Otero }\end{array}$ & 9 1/2 HEMINAS & - & $\begin{array}{l}24 \text { CARGAS } \\
2 \text { COLONOS } \\
\end{array}$ & ENCINAS & $\begin{array}{l}5 \text { 1/3 HEMINAS DE } \\
\text { TRIGO }\end{array}$ \\
\hline & Otro solar & $\begin{array}{c}10 \frac{1}{2} / 2 \\
\text { HEMINAS }\end{array}$ & $\begin{array}{c}24 \text { AZUMBRES } \\
1 \text { CARGA }\end{array}$ & 18 CARGAS & $\dot{¿} ?$ & $\begin{array}{l}6 \text { 1/4 HEMINAS DE TRIGO } \\
\text { Y } 1 \text { AZUMBRE DE VINO }\end{array}$ \\
\hline
\end{tabular}




\begin{tabular}{|c|c|c|c|c|c|c|}
\hline & $\begin{array}{l}\text { Cabo la casa de } \\
\text { Teresa }\end{array}$ & 4 HEMINAS & - & $\begin{array}{c}1 \text { CARGAS } \\
3 \\
\text { MOSTELAS }\end{array}$ & - & $11 \frac{1}{4}$ HEMINAS DE TRIGO \\
\hline & $\begin{array}{l}\text { Tras la casa de } \\
\text { Alonso de Tanarrio }\end{array}$ & - & 32 AZUMBRES & - & - & 2 HEMINAS DE TRIGO \\
\hline & Solar del Cabrero & 7 HEMINAS & - & - & - & $\begin{array}{l}21 / 2 \text { HEMINAS DE TRIGO } \\
\text { Y } 2 \text { AZUMBRES DE } \\
\text { VINO }\end{array}$ \\
\hline & Otro solar & 2 HEMINAS & - & 12 CARGAS & - & $21 / 2$ HEMINAS DE TRIGO \\
\hline LUARNA & & - & - & 12 CARGAS & - & $\begin{array}{l}1 \text { HEMINA DE TRIGO Y } \\
1 \text { GALLINA }\end{array}$ \\
\hline \multirow[t]{2}{*}{ BÁRCENA } & Dos solares & $\begin{array}{l}3 \text { HEMINAS } \\
3 \text { HEMINAS }\end{array}$ & - & 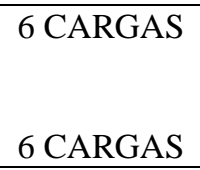 & $\begin{array}{l}? ? \\
\dot{¿} ?\end{array}$ & $\begin{array}{l}2 \text { HEMINAS DE TRIGO, } 1 \\
\text { GALLINA, } 2 \\
\text { MARAVEDIES Y } 1 / 2 \\
\text { BLANCA VIEJA }\end{array}$ \\
\hline & Otro solar & 8 HEMINAS & - & - & - & $\begin{array}{l}4 \text { HEMINAS DE TRIGO Y } \\
1 \text { GALLINA }\end{array}$ \\
\hline DOBARGANES & & - & $\dot{¿} ?$ & - & - & 10 HEMINAS DE TRIGO \\
\hline \multirow[t]{2}{*}{ DOBRES } & & 49 HEMINAS & - & 42 CARGAS & - & $\begin{array}{l}\text { 14 HEMINAS DE TRIGO } \\
\text { Y } 14 \text { DE CENTENO }\end{array}$ \\
\hline & $\begin{array}{l}\text { Solar de Juan de } \\
\text { Valcayo }\end{array}$ & 6 HEMINAS & - & - & - & 4 HEMINAS DE TRIGO \\
\hline LA VEGA & & 40 HEMINAS & - & - & - & 24 HEMINAS DE TRIGO \\
\hline ARMAÑO & & 4 HEMINAS & 8 AZUMBRES & $\dot{¿} ?$ & - & $\begin{array}{l}2 \text { HEMINAS DE TRIGO Y } \\
2 \text { DE CENTENO }\end{array}$ \\
\hline \multirow[t]{3}{*}{ TAMA } & En el barrio de Suso & 2 HEMINAS & - & - & - & 1/2 GALLINA Y 1 1⁄2 PAN \\
\hline & En el barrio de villa & 8 HEMINAS & 2-3 CARGAS & - & - & $\begin{array}{l}4 \text { HEMINAS DE TRIGO Y } \\
4 \text { AZUMBRES DE VINO }\end{array}$ \\
\hline & Otro solar & - & $\begin{array}{c}136 \\
\text { AZUMBRES }\end{array}$ & - & - & $\begin{array}{l}4 \text { HEMINAS DE TRIGO Y } \\
8 \text { AZUMBRES DE VINO }\end{array}$ \\
\hline ALIEZO & $\begin{array}{l}\text { Solar de la Calleja } \\
\text { Solar en Hondón de } \\
\text { villa }\end{array}$ & $\begin{array}{l}8 \text { HEMINAS } \\
3 \text { HEMINAS }\end{array}$ & $\begin{array}{c}232 \\
\text { AZUMBRES } \\
144 \\
\text { AZUMBRES }\end{array}$ & - & - & $\begin{array}{l}3 \text { HEMINAS DE TRIGO, } 3 \\
\text { DE CENTENO, } 4 \\
\text { AZUMBRES DE VINO Y } \\
1 \text { GALLINA }\end{array}$ \\
\hline
\end{tabular}




\begin{tabular}{|c|c|c|c|c|c|c|}
\hline & Otro solar & 2 HEMINAS & $\begin{array}{c}144 \\
\text { AZUMBRES }\end{array}$ & - & - & $\begin{array}{l}3 \text { HEMINAS DE TRIGO Y } \\
4 \text { AZUMBRES DE VINO }\end{array}$ \\
\hline & En la aldea del Valle & 1 HEMINA & 48 AZUMBRES & - & - & $\begin{array}{l}11 / 2 \text { HEMINAS DE } \\
\text { TRIGO, } 11 \frac{1}{2} \text { DE } \\
\text { CENTENO Y } 4 \\
\text { AZUMBRES DE VINO }\end{array}$ \\
\hline \multirow[t]{2}{*}{ OJEDO } & $\begin{array}{l}\text { Solar en Fondón de } \\
\text { Villa }\end{array}$ & 4 HEMINAS & $\begin{array}{c}256 \\
\text { AZUMBRES }\end{array}$ & $1 / 2$ CARGA & $\dot{¿} ?$ & $\begin{array}{l}3 \text { HEMINAS DE TRIGO, } 3 \\
\text { DE CENTENO Y } 8 \\
\text { AZUMBRES DE VINO }\end{array}$ \\
\hline & Solar del Otero & 30 HEMINAS & $\begin{array}{c}1808 \\
\text { AZUMBRES }\end{array}$ & $\begin{array}{c}71 / 2 \\
\text { CARGAS }\end{array}$ & CEREZOS & $\begin{array}{l}3 \text { HEMINAS DE TRIGO, } 3 \\
\text { DE CENTENO Y } 8 \\
\text { AZUMBRES DE VINO }\end{array}$ \\
\hline \multirow[t]{3}{*}{ FRAMA } & Solar de la Puente & $\begin{array}{c}171 / 2 \\
\text { HEMINAS }\end{array}$ & $\begin{array}{c}272 \\
\text { AZUMBRES }\end{array}$ & - & - & $\begin{array}{l}41 / 2 \text { HEMINAS DE } \\
\text { TRIGO, } 41 / 2 \text { DE } \\
\text { CENTENO, } 12 \\
\text { AZUMBRES DE VINO Y } \\
3 \text { BLANCAS }\end{array}$ \\
\hline & Solar del Prado & 39 HEMINAS & $\begin{array}{c}432 \\
\text { AZUMBRES }\end{array}$ & - & - & $\begin{array}{l}3 \text { HEMINAS DE TRIGO, } 3 \\
\text { DE CENTENO, } 16 \\
\text { AZUMBRES Y } 2 \\
\text { MARAVEDIES }\end{array}$ \\
\hline & $\begin{array}{l}\text { Solar de Martín } \\
\text { García }\end{array}$ & 7 HEMINAS & $\begin{array}{c}328 \\
\text { AZUMBRES }\end{array}$ & - & - & $\begin{array}{l}3 \text { HEMINAS DE TRIGO, } 3 \\
\text { DE CENTENO, } 8 \\
\text { AZUMBRES DE VINO Y } \\
1 \text { MARAVEDÍ }\end{array}$ \\
\hline \multirow[t]{3}{*}{ CAMBARCO } & Solar de las Obesas & 2 HEMINAS & 60 AZUMBRES & - & $\dot{¿} ?$ & $\begin{array}{l}3 \text { HEMINAS DE TRIGO Y } \\
3 \text { DE CEBADA }\end{array}$ \\
\hline & Solar del Palacio & $31 / 2$ HEMINAS & $\begin{array}{c}120 \\
\text { AZUMBRES }\end{array}$ & - & $\dot{¿} ?$ & $\begin{array}{l}3 \text { HEMINAS DE TRIGO Y } \\
3 \text { DE CEBADA }\end{array}$ \\
\hline & Solar de la Cortina & 2 HEMINAS & - & - & - & 4 HEMINAS DE CEBADA \\
\hline CAHECHO & & $\begin{array}{c}361 / 2 \\
\text { HEMINAS }\end{array}$ & 48 AZUMBRES & 1 CARGA & - & 8 HEMINAS DE TRIGO \\
\hline TREVIÑO & & 13 HEMINAS & - & 48 CARGAS & - & $\begin{array}{l}6 \text { HEMINAS DE TRIGO Y } \\
6 \text { MARAVEDIES }\end{array}$ \\
\hline ENTERRÍA & & - & 96 AZUMBRES & - & - & $\begin{array}{l}1 \text { HEMINA DE TRIGO Y } \\
1 \text { GALLINA }\end{array}$ \\
\hline
\end{tabular}




\begin{tabular}{|c|c|c|c|c|c|c|}
\hline & Otro solar & 12 HEMINAS & - & 12 CARGAS & $\dot{d} ?$ & $\begin{array}{l}2 \text { HEMINAS DE TRIGO, } 1 \\
\text { GALLINA Y } 3 \text { DINEROS }\end{array}$ \\
\hline \multirow[t]{14}{*}{ LEBEÑA } & Solar del Ojo & - & - & 5 CARGAS & - & $\begin{array}{l}4 \text { HEMINAS DE TRIGO Y } \\
5 \text { BLANCAS VIEJAS }\end{array}$ \\
\hline & Solar de Vazna & 3 HEMINAS & 96 AZUMBRES & - & - & $\begin{array}{l}8 \text { HEMINAS DE TRIGO, } \\
16 \text { AZUMBRES Y } 5 \\
\text { BLANCAS VIEJAS } \\
\end{array}$ \\
\hline & Solar de la Torre & - & 24 AZUMBRES & 2 COLONOS & - & $\begin{array}{l}5 \text { HEMINAS DE TRIGO Y } \\
5 \text { BLANCAS VIEJAS }\end{array}$ \\
\hline & $\begin{array}{l}\text { Solar en la aldea de } \\
\text { Quintanilla }\end{array}$ & - & 3 AZUMBRES & - & - & $\begin{array}{l}1 / 2 \text { HEMINA DE TRIGO, } 1 / 2 \\
\text { DE CEBADA Y 2,5 } \\
\text { BLANCAS VIEJAS }\end{array}$ \\
\hline & Encima villa & 2 HEMINAS & - & - & - & 4 HEMINAS DE TRIGO \\
\hline & Solar de la Herrería & - & 64 AZUMBRES & - & - & $\begin{array}{l}4 \text { HEMINAS DE TRIGO, } 4 \\
\text { AZUMBRES Y } 5 \\
\text { BLANCAS VIEJAS }\end{array}$ \\
\hline & En el Pontón & 4 HEMINAS & 64 AZUMBRES & - & - & $\begin{array}{l}4 \text { HEMINAS DE TRIGO Y } \\
5 \text { BLANCAS VIEJAS }\end{array}$ \\
\hline & En tras el Ojuelo & $1 / 2$ HEMINA & 40 AZUMBRES & - & - & $\begin{array}{l}3 \text { 3/4 HEMINAS DE } \\
\text { TRIGO, } 3 \text { AZUMBRES DE } \\
\text { VINO Y } 2 \text { MARAVEDIES }\end{array}$ \\
\hline & En Hondón de villa & - & 16 AZUMBRES & - & - & $\begin{array}{l}4 \text { HEMINAS DE TRIGO Y } \\
5 \text { BLANCAS VIEJAS }\end{array}$ \\
\hline & En el Arenal & 7 HEMINAS & 64 AZUMBRES & - & - & $\begin{array}{l}8 \text { HEMINAS DE TRIGO Y } \\
5 \text { BLANCAS VIEJAS }\end{array}$ \\
\hline & $\begin{array}{l}\text { Solar de Juan } \\
\text { González }\end{array}$ & 4 HEMINAS & 80 AZUMBRES & - & - & $\begin{array}{l}5 \text { HEMINAS DE TRIGO Y } \\
5 \text { BLANCAS VIEJAS }\end{array}$ \\
\hline & Solar Viejo & - & 32 AZUMBRES & - & - & $\begin{array}{l}11 / 2 \text { HEMINA DE TRIGO, } \\
2 \text { DE CEBADA Y } 5 \\
\text { BLANCAS VIEJAS }\end{array}$ \\
\hline & En Cimavilla & - & 96 AZUMBRES & - & - & $\begin{array}{l}5 \text { HEMINAS DE TRIGO Y } \\
5 \text { BLANCAS VIEJAS }\end{array}$ \\
\hline & $\begin{array}{l}\text { Sobre el nogal de la } \\
\text { Torre }\end{array}$ & - & 48 AZUMBRES & - & - & $\begin{array}{l}2 \text { HEMINAS DE TRIGO, } 2 \\
\text { DE CEBADA, } 6 \\
\text { AZUMBRES Y } 5 \\
\text { BLANCAS VIEJAS }\end{array}$ \\
\hline
\end{tabular}




\begin{tabular}{|c|c|c|c|c|c|c|}
\hline & Otro solar & - & 72 AZUMBRES & - & - & $\begin{array}{l}5 \text { HEMINAS DE TRIGO Y } \\
5 \text { BLANCAS VIEJAS }\end{array}$ \\
\hline & En Hondón de villa & 2 HEMINAS & 28 AZUMBRES & - & $\dot{i} ?$ & 4 HEMINAS DE TRIGO \\
\hline & En tras Quintanilla & - & 20 AZUMBRES & - & - & 4 HEMINAS DE TRIGO \\
\hline & En tras el Ojuelo & 2 HEMINAS & 3 AZUMBRES & - & $\begin{array}{c}2 \text { NOGALES } \\
\dot{¿} ?\end{array}$ & 9 HEMINAS DE TRIGO \\
\hline & Medio solar & - & 96 AZUMBRES & 3 CARGAS & - & $\begin{array}{l}4 \text { HEMINAS DE TRIGO Y } \\
5 \text { BLANCAS }\end{array}$ \\
\hline \multirow[t]{8}{*}{ ENTERRIAS } & $\begin{array}{l}\text { Solar de Fernando } \\
\text { de Enterrías, el viejo }\end{array}$ & 14 HEMINAS & - & 18 CARGAS & - & $\begin{array}{l}4 \text { HEMINAS DE TRIGO, } 1 \\
\text { GALLINA Y SU PARTE } \\
\text { DEL YANTAR }\end{array}$ \\
\hline & Solar del Pumar & 2 HEMINAS & - & - & - & $\begin{array}{l}1 \text { HEMINA DE TRIGO, } 1 \\
\text { GALLINA Y SU PARTE } \\
\text { DEL YANTAR }\end{array}$ \\
\hline & Solar de la presa & 3 HEMINAS & - & - & - & $\begin{array}{l}1 \text { HEMINA DE TRIGO, } 1 \\
\text { GALLINA Y SU PARTE } \\
\text { DEL YANTAR }\end{array}$ \\
\hline & En el Cantil & 6 HEMINAS & - & - & - & $\begin{array}{l}1 \text { HEMINA DE TRIGO, } 1 \\
\text { GALLINA Y SU PARTE } \\
\text { DEL YANTAR }\end{array}$ \\
\hline & Solar de la Tapia & 1 HEMINA & - & - & - & $\begin{array}{l}3 \text { HEMINAS DE } \\
\text { CENTENO Y SU PARTE } \\
\text { DEL YANTAR }\end{array}$ \\
\hline & Solar de la Puerta & 3 HEMINAS & - & - & - & $\begin{array}{l}3 \text { HEMINAS DE } \\
\text { CENTENO Y SU PARTE } \\
\text { DEL YANTAR }\end{array}$ \\
\hline & $\begin{array}{l}\text { Solar de Juan Prieto } \\
\text { Solar de la Fragua } \\
\text { Solar del Horquero }\end{array}$ & 17 HEMINAS & - & - & - & $\begin{array}{l}10 \text { HEMINAS DE } \\
\text { CENTENO, } 1 \text { DE TRIGO, } \\
1 \text { GALLINA Y SU PARTE } \\
\text { DEL YANTAR }\end{array}$ \\
\hline & Otro solar & - & $\begin{array}{c}120 \\
\text { AZUMBRES }\end{array}$ & - & - & 8 HEMINAS DE TRIGO \\
\hline BEJES & & 10 HEMINAS & - & $\begin{array}{c}6 \text { CARGAS } \\
10 \\
\text { COLONOS } \\
\end{array}$ & - & 150 MARAVEDIES \\
\hline PENDES & & 12 HEMINAS & $\begin{array}{c}384 \\
\text { AZUMBRES }\end{array}$ & $\begin{array}{c}1 \frac{1 / 2}{2} \\
\text { CARGAS }\end{array}$ & - & $\begin{array}{l}10 \text { HEMINAS DE TRIGO } \\
\text { Y } 14 \text { AZUMBRES DE }\end{array}$ \\
\hline
\end{tabular}




\begin{tabular}{|l|c|c|c|c|c|l|}
\hline & & & & 4 COLONOS & & VINO \\
\hline REDONDO & & - & - & $\begin{array}{c}\text { 6 CARGAS } \\
1 \\
\text { ENTUERTA }\end{array}$ & $\begin{array}{c}\text { 1 HEMINA DE } \\
\text { LINO }\end{array}$ & $\begin{array}{l}\text { 8 HEMINAS DE } \\
\text { CENTENO }\end{array}$ \\
\cline { 2 - 6 } & & - & - & $\begin{array}{c}18 \text { CARGAS } \\
1 \\
\text { ENTUERTA }\end{array}$ & $\begin{array}{c}1 \frac{1}{2} \text { HEMINA } \\
\text { DE LINO }\end{array}$ & $\begin{array}{l}\text { 8 HEMINAS DE } \\
\text { CENTENO }\end{array}$ \\
\hline ROSCALES & & & & 120 & & $\begin{array}{l}\text { 40 HEMINAS DE TRIGO } \\
\text { Y 24 DE CEBADA }\end{array}$ \\
\hline
\end{tabular}




\section{SOLARES EN MIESES}

\begin{tabular}{|c|c|c|c|c|}
\hline TENENTE & 1499 & 1515 & 1538 & RENTA \\
\hline $\begin{array}{l}\text { 1499: TERESA, la Casera } \\
1515 \text { y 1538: GARCÍA } \\
\text { DE TERESA }\end{array}$ & $\begin{array}{l}\text { 1 CAMPO que está junto con } \\
\text { el dicho solar con su arboleda } \\
1 \text { TIERRA en el Cascajal } \\
\text { 1SESTERO DE TRIGO } \\
1 \text { VIÑA en el Préstamo } \\
6 \text {-7-MIEDROS DE VINO } \\
\text { NO FIGURA } \\
1 \text { VIÑA en el Valladar } \\
2 \text { MIEDROS DE VINO } \\
1 \text { VIÑA en hondon del } \\
\text { Valladar } 2 \text { CÁNTARAS DE VINO } \\
1 \text { CASAR junto con el dicho } \\
\text { solar que es ahora Huerto } \\
\text { ¿? }\end{array}$ & $\begin{array}{l}\text { 1VIÑA en Viña Mayor } \\
6 \text { a } 7 \text { MIEDRO DE VINO } \\
1 \text { VIÑA en el Valladar } \\
1 \text { CARGA DE UVAS }\end{array}$ & 2 CÁNTARAS DE VINO & $\begin{array}{l}\text { PAGA DE INFURCION } 10 \\
\text { HEMINAS DE TRIGO, } 14 \\
\text { AZUMBRES DE VINO, } 3 \\
\text { HUEBRAS DE BUEYES } \\
\text { Y } 1 \text { GALLINA (que paga } \\
\text { por el dicho casar). HA DE } \\
\text { QUEDAR EN } 1 \\
\text { HEREDERO Y NO MAS }\end{array}$ \\
\hline $\begin{array}{l}\text { ARMENTAL. El solar de } \\
\text { Catalina } \\
\text { 1499: RODRIGO DE LA } \\
\text { FUENTE }\end{array}$ & $\begin{array}{l}1 \text { TIERRA al Pumar } \\
1 \text { HEMINA DE TRIGO }\end{array}$ & 1 SESTERO DE TRIGO & & $\begin{array}{l}\text { PAGA DE INFURCIÓN } 3 \\
\text { PANES Y } 1 \text { GALLINA }\end{array}$ \\
\hline
\end{tabular}




\begin{tabular}{|c|c|c|c|c|}
\hline $\begin{array}{l}1515 \text { y 1538: JUAN DE } \\
\text { RODRIGO }\end{array}$ & & & & \\
\hline $\begin{array}{l}\text { ARMENTAL. El solar de } \\
\text { Ferrand de Baró } \\
\text { 1499 Y 1515: FERRAND } \\
\text { BARÓ } \\
\text { 1538: HEREDEROS DE } \\
\text { FERNANDO DE BARÓ }\end{array}$ & $\begin{array}{l}1 \text { HUERTO bajo el solar } \\
1 \text { SESTERO DE LINAZA } \\
1 \text { VIÑA en so Casa } \\
3 \text { MIEDROS DE VINO } \\
1 \text { VIÑA en el Pedregoso } \\
4 \text { CÁNTARAS DE VINO } \\
2 \text { HAZAS DE TIERRA en } \\
\text { Xostrada } \\
1 \text { CUARTO DE TRIGO } \\
\text { NO FIGURA }\end{array}$ & $\begin{array}{l}1 \text { VIÑA y PRADO en so } \\
\text { Casa } \\
\text { 1CARRO DE YERBA } \\
4 \text { MIEDROS DE VINO } \\
3 \text { MIEDROS DE VINO } \\
2 \text { HAZAS DE VIÑAS en } \\
\text { Xostrada } \\
3 \text { MIEDROS DE VINO } \\
\text { NO FIGURA }\end{array}$ & $\begin{array}{l}\text { 3-4 CÁNTARAS VINO } \\
1 \text { VIÑA en Xostrada } \\
2 \text { MIEDROS DE VINO }\end{array}$ & $x^{2}$ \\
\hline $\begin{array}{l}\text { 1499: Solar de la } \\
\text { Corralona } \\
\text { 1515: El solar de Gómez } \\
\text { de Otero } \\
\text { 1499: GÓMEZ DE } \\
\text { OTERO } \\
\text { 1515: JUANA DE } \\
\text { GÓMEZ y ELVIRA DE } \\
\text { FLORANES } \\
\text { 1538: ALONSO GÓMEZ }\end{array}$ & $\begin{array}{l}2 \text { TIERRAS en Alzapierna } \\
6 \text { HEMINAS DE TRIGO } \\
1 \text { HUERTO } \\
1 \text { SESTERO DE LINAZA } \\
1 \text { HUERTO en hondon de } \\
\text { villa } \\
1 \text { HEMINA DE LINAZA } \\
1 \text { VIÑA en Viña Mayor } \\
4 \text { CÁNTARAS DE VINO }\end{array}$ & $\begin{array}{l}2 \text { VIÑAS en Alzapierna } \\
2 \text { MIEDROS DE VINO } \\
1 \text { MIEDRO DE VINO }\end{array}$ & $\begin{array}{l}\text { NO FIGURA } \\
\text { NO FIGURA }\end{array}$ & \\
\hline
\end{tabular}




\begin{tabular}{|c|c|c|c|c|}
\hline DE MIESES & $\begin{array}{l}1 \text { VIÑA en Llandeçilla } \\
4 \text { CÁNTARAS DE VINO } \\
1 \text { VIÑA en la Molina } \\
1 \text { CÁNTARA DE VINO } \\
\text { HAZA DE VIÑA en Llan de } \\
\text { Otero } \\
1 \text { CÁNTARA DE VINO } \\
1 \text { VIÑA so la Orden } \\
1 \text { MIEDRO DE VINO } \\
1 \text { TIERRA en Cerinama } \\
6 \text { HEMINAS DE TRIGO } \\
1 \text { TIERRA en el Cuerano } \\
1 \text { CUARTO DE TRIGO } \\
1 \text { VIÑA en so Carrera } \\
1 \text { MIEDRO DE VINO } \\
1 \text { VIÑA en Viña Mayor } \\
1 \text { MIEDRO DE VINO }\end{array}$ & 2 CARGAS DE UVAS & $\begin{array}{l}\text { NO FIGURA } \\
\text { NO FIGURA } \\
\text { NO FIGURA }\end{array}$ & $\begin{array}{l}\text { PAGA DE INFURCIÓN } 3 \\
\text { CUARTOS DE TRIGO, } 14 \\
\text { AZUMBRES DE VINO Y } \\
11 \frac{1}{2} \text { HUEBRAS DE } \\
\text { BUEYES }\end{array}$ \\
\hline $\begin{array}{l}\text { ARMENTAL Solar de la } \\
\text { Cortina } \\
\text { 1499: JUANA DE } \\
\text { CASILLAS y herederos } \\
\text { de FERRANDO DE } \\
\text { VALLINAS } \\
\text { 1515: SANCHO DE } \\
\text { CASILLAS DE MIESES } \\
\text { y JUAN DE LA }\end{array}$ & $\begin{array}{l}1 \text { HUERTO delante del solar } \\
1 \text { SESTERO DE LINAZA } \\
1 \text { VIÑA en Llan de Viñas } \\
\text { 2-3-MIEDROS DE VINO } \\
1 \text { VIÑA en Viña Mayor } \\
1 \text { MIEDRO DE VINO }\end{array}$ & $\begin{array}{l}1 \text { FANEGA DE LINAZA } \\
4 \text { MIEDROS DE VINO } \\
2 \text { MIEDROS DE VINO }\end{array}$ & & $\begin{array}{l}\text { PAGA DE INFURCION } 10 \\
\text { HEMINAS DE TRIGO, } 14 \\
\text { AZUMBRES DE VINO y } \\
3 \text { HUEBRAS DE BUEYES }\end{array}$ \\
\hline
\end{tabular}




\begin{tabular}{|c|c|c|c|c|}
\hline $\begin{array}{l}\text { CALLEJA } \\
\text { 1538: PEDRO DE } \\
\text { TREVIA y TORIBIO, } \\
\text { HIJO DE SANCHO DE } \\
\text { CASILLAS }\end{array}$ & & & & \\
\hline $\begin{array}{l}\text { MIESES. Solar de } \\
\text { Gonzalo de Baró } \\
\text { 1499: GONZALO DE } \\
\text { BARÓ, merino. } \\
\text { 1515: Hijos de } \\
\text { GONZALO DE BARÓ } \\
\text { 1538: JUAN DEL } \\
\text { MERINO Y GARCÍA DE } \\
\text { ENTERRIA, el mozo. }\end{array}$ & $\begin{array}{l}1 \text { TIERRA junto con el solar } \\
2 \text { FANEGAS DE TRIGO }\end{array}$ & 5 CUARTOS DE TRIGO & & $\begin{array}{l}\text { PAGA DE INFURCIÓN } 1 \\
\text { FANEGA DE TRIGO }\end{array}$ \\
\hline 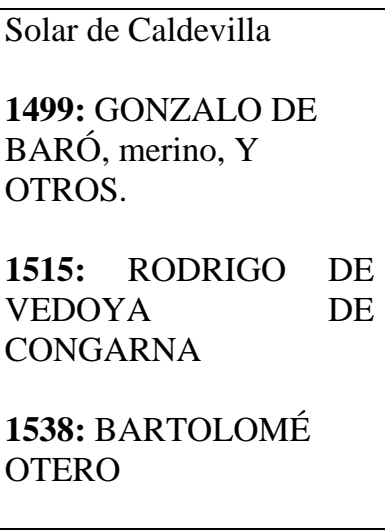 & $\begin{array}{l}1 \text { VIÑA en la Pasadilla que es } \\
\text { el portillo de Viña Mayor } \\
3 \text { MIEDROS DE VINO } \\
1 \text { VIÑA en so Otero } \\
1 \text { MIEDRO DE VINO } \\
1 \text { TIERRA en Xostrada } \\
1 \text { CUARTO DE TRIGO } \\
1 \text { PRADO en la aldea de } \\
\text { Mieses } \\
11 \frac{1}{2} \text { CARRO DE YERBA }\end{array}$ & $\begin{array}{l}5 \text { MIEDROS DE VINO } \\
2 \text { MIEDROS DE VINO }\end{array}$ & 3-4 MIEDROS DE VINO & $\begin{array}{l}\text { PAGA DE INFURCION } 10 \\
\text { HEMINAS DE TRIGO, } 14 \\
\text { AZUNBRES DE VINO Y } 3 \\
\text { HUEBRAS DE BUEYES. } \\
\text { HA DE QUEDAR EN } 1 \\
\text { HEREDERO Y NO MAS. }\end{array}$ \\
\hline $\begin{array}{l}\text { Solar de Hernando de la } \\
\text { Posada } \\
\text { 1499: ALONSO DEL } \\
\text { VALLE y MARÍA, mujer } \\
\text { de JUAN DE LA }\end{array}$ & $\begin{array}{l}1 \text { HUERTO junto al solar } \\
1 \text { HEMINA DE LINAZA } \\
1 \text { VIÑA en Tabejana } \\
2 \text { MIEDROS DE VINO }\end{array}$ & 11/2 HEMINA LINAZA & 3 MIEDROS DE VINO & $\begin{array}{l}\text { PAGA DE INFURCIÓN } 10 \\
\text { HEMINAS DE TRIGO, } 14 \\
\text { AZUMBRES DE VINO y }\end{array}$ \\
\hline
\end{tabular}




\begin{tabular}{|c|c|c|c|c|}
\hline $\begin{array}{l}\text { POSADA. } \\
\text { 1515: ALONSO DEL } \\
\text { VALLE y MARÍA DE } \\
\text { LA POSADA } \\
\text { 1538: FERNANDO DE } \\
\text { LA POSADA }\end{array}$ & $\begin{array}{l}1 \text { VIÑA en Canpijon } \\
\text { 1-2 MIEDROS DE VINO } \\
1 \text { TIERRA en Cerinama } \\
1 \text { TIERRA en Cerinama } \\
3 \text { CUARTOS DE PAN }\end{array}$ & 4-8 CÁNTARAS VINO & 2 MIEDROS DE VINO & $\begin{array}{l}3 \text { HUEBRAS DE } \\
\text { BUEYES. }\end{array}$ \\
\hline $\begin{array}{l}\text { Solar de Juan Herrero con } \\
\text { su corral. En el apeo de } \\
\text { 1499 son dos solares. } \\
\text { 1499: JUAN HERRERO } \\
\text { (los dos solares) } \\
\text { 1515: PEDRO DE } \\
\text { CASILLAS } \\
\text { 1538: PEDRO } \\
\text { VALDEÓN }\end{array}$ & $\begin{array}{l}1 \text { HUERTO delante de la } \\
\text { casa } \\
1 \text { HEMINA DE LINAZA } \\
1 \text { TIERRA en Valdevallino } \\
1 \text { SESTERO DE TRIGO } \\
1 \text { TIERRA en Valdevallino } \\
1 \text { SESTERO DE TRIGO } \\
1 \text { PRADO en Valdevallino } \\
1 \text { CARGA DE YERBA } \\
\text { NO FIGURA } \\
\text { NO FIGURA } \\
\text { NO FIGURA }\end{array}$ & $\begin{array}{l}1 \text { TIERRA en las casas } \\
1 \text { SESTERO DE TRIGO } \\
1 \text { TIERRA en las casas } \\
\dot{¿} ? \\
1 \text { TIERRA en Valdeviñón } \\
1 \text { HEMINA DE TRIGO }\end{array}$ & $\begin{array}{l}3 \text { CARGAS DE YERBA } \\
1 \text { HEMINA DE PAN }\end{array}$ & $\begin{array}{l}\text { 1499: PAGA DE } \\
\text { INFURCION } 10 \\
\text { HEMINAS DE TRIGO Y } 1 \\
\text { GALLINA (Entre los dos } \\
\text { solares) } \\
\text { 1515 Y 1538: } 1 \text { FANEGA } \\
\text { DE TRIGO Y } 1 \text { GALLINA. } \\
\text { 1515: HA DE QUEDAR } \\
\text { SIEMPRE EN DOS } \\
\text { HEREDEROS Y NO MAS. } \\
\text { 1538: EN UN HEREDERO } \\
\text { Y NO MAS. }\end{array}$ \\
\hline
\end{tabular}




\begin{tabular}{|c|c|c|c|c|}
\hline & $\begin{array}{l}\text { NO FIGURA } \\
\text { NO FIGURA } \\
\text { NO FIGURA } \\
\text { NO FIGURA } \\
\text { NO FIGURA } \\
\text { NO FIGURA } \\
\text { NO FIGURA }\end{array}$ & $\begin{array}{l}1 \text { TIERRA en la Molina } \\
3 \text { HEMINAS DE TRIGO } \\
1 \text { PRADO en Fuentes } \\
1 \text { CARGA DE YERBA } \\
\text { NO FIGURA } \\
\text { NO FIGURA } \\
\text { NO FIGURA } \\
\text { NO FIGURA } \\
\text { NO FIGURA }\end{array}$ & $\begin{array}{l}1 \text { TIERRA en la Molina } \\
1 \text { SESTERO DE TRIGO } \\
1 \text { TIERRA Valdeballino } \\
1 \text { HEMINA DE PAN } \\
1 \text { TIERRA Valdeballino } \\
1 \text { HEMINA DE PAN } \\
1 \text { TIERRA Valdeballino } \\
1 \text { HEMINA DE PAN } \\
1 \text { HAZA DE PRADO en } \\
\text { Valdeballino } \\
1 \text { MOSTELA de YERBA }\end{array}$ & \\
\hline $\begin{array}{l}\text { 1499: Solar de Antón } \\
\text { 1515 y 1538: Solar de } \\
\text { García de Enterría. } \\
\text { 1499: GARCÍA DE } \\
\text { ENTERRÍA } \\
\text { 1515: MAYOR GARCÍA, } \\
\text { mujer de GARCÍA DE } \\
\text { ENTERRÍA } \\
\text { 1538: GARCÍA DE } \\
\text { ENTERRÍA y } \\
\text { HEREDEROS DE JUAN }\end{array}$ & $\begin{array}{l}1 \text { HUERTO junto al solar } \\
3 \text { HEMINAS DE LINAZA } \\
1 \text { TIERRA en el prado de } \\
\text { Mieses } \\
1 \text { CUARTO DE TRIGO } \\
1 \text { VIÑA en el Pedregoso } \\
5-6 \text { MIEDROS DE VINO } \\
2 \text { PRADOS en la Prada de } \\
\text { Argüébanes } \\
11 \frac{1}{2} \text { CARRO }\end{array}$ & $\begin{array}{l}5 \text { HEMINAS de LINAZA } \\
\text { NO FIGURA }\end{array}$ & 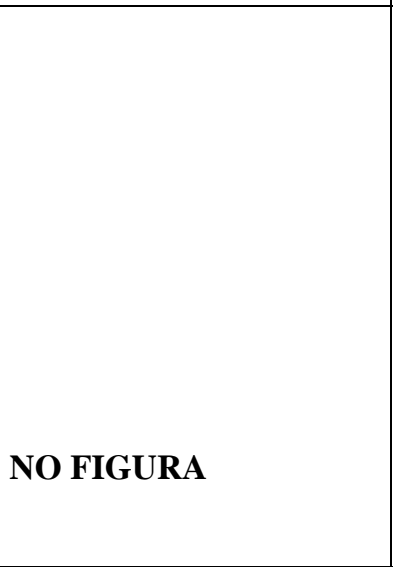 & $\begin{array}{l}\text { PAGA DE INFURCION } 10 \\
\text { HEMINAS DE TRIGO, } 14 \\
\text { AZUMBRES DE VINO Y } \\
3 \text { HUEBRAS DE } \\
\text { BUEYES. }\end{array}$ \\
\hline
\end{tabular}




\begin{tabular}{|c|c|c|c|c|}
\hline $\begin{array}{l}\text { RODRÍGUEZ, SU } \\
\text { HERMANO }\end{array}$ & NO FIGURA & NO FIGURA & $\begin{array}{l}1 \text { HAZA DE VIÑA en } \\
\text { Tabejana } \\
2 \text { CÁNTARAS DE VINO }\end{array}$ & \\
\hline $\begin{array}{l}\text { Solar de la Herren } \\
\text { 1499: JUAN GÓMEZ y } \\
\text { JUAN DE LAMASÓN } \\
\text { 1515: TORIBIO DE } \\
\text { BARÓ DE TURIENO y } \\
\text { JUAN GÓMEZ DE } \\
\text { MIESES } \\
\text { 1538: INÉS GONZÁLEZ } \\
\text { DE TURIENO e ISABEL } \\
\text { DE MIESES }\end{array}$ & $\begin{array}{l}1 \text { TIERRA en la Vega de } \\
\text { Turieno } \\
3 \text { CUARTOS DE TRIGO } \\
1 \text { VIÑA en so Otero } \\
4 \text { MIEDROS DE VINO } \\
1 \text { VIÑA en Oteruelo } \\
3 \text { MIEDROS DE VINO } \\
1 \text { VIÑA en Olinas } \\
2 \text { MIEDROS DE VINO } \\
1 \text { VIÑA en Camares } \\
2 \text { MIEDROS DE VINO } \\
1 \text { VIÑA en la Valleja de Viña } \\
\text { Mayor } \\
2 \text { MIEDROS DE VINO } \\
1 \text { TIERRA encima de las } \\
\text { viñas de Viña Mayor } \\
\text { HEMINA DE TRIGO } \\
1 \text { VIÑA en Viña Mayor } \\
2 \text { MIEDROS DE VINO }\end{array}$ & $\begin{array}{l}2 \text { FANEGAS DE TRIGO } \\
3 \text { MIEDROS DE VINO } \\
3 \text { MIEDROS DE VINO } \\
3 \text { MIEDROS DE VINO }\end{array}$ & 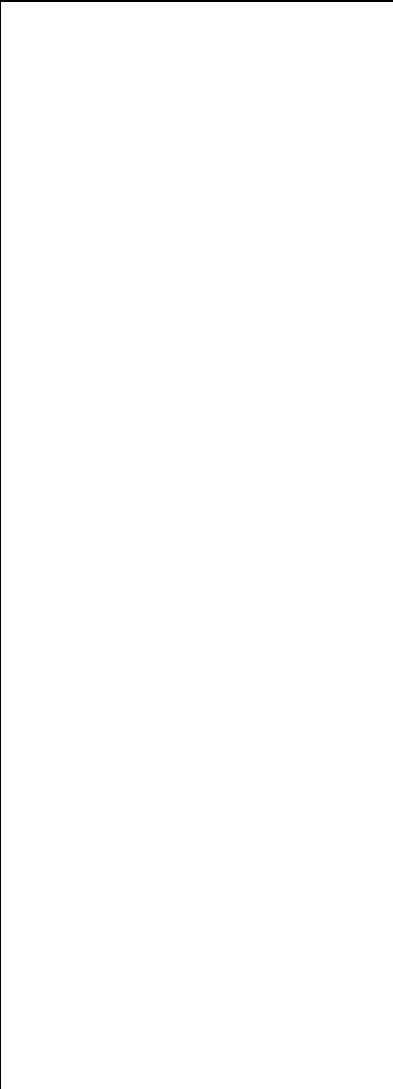 & $\begin{array}{l}\text { PAGA DE INFURCION } 15 \\
\text { HEMINAS DE TRIGO } 21 \\
\text { AÇUNBRES DE VINO Y } \\
4 \text { HUEBRAS Y } 1 / 2 \text { DE } \\
\text { BUEYES. HA DE } \\
\text { QUEDAR EN DOS } \\
\text { HEREDEROS Y NO MAS. }\end{array}$ \\
\hline & $\begin{array}{l}\text { NO FIGURA } \\
1 \text { VIÑA en la Escuentra }\end{array}$ & 1 HUERTO cabo el solar & NO FIGURA & \\
\hline
\end{tabular}




\begin{tabular}{|c|c|c|c|c|}
\hline & $\begin{array}{l}1 \text { MIEDRO DE VINO } \\
1 \text { TIERRA en Callejo } \\
1 \text { SESTERO DE TRIGO } \\
4 \text { NOZALES en hondon de la } \\
\text { riega }\end{array}$ & & 2 MIEDROS DE VINO & \\
\hline $\begin{array}{l}\text { 1499 y 1515: SANCHO } \\
\text { RUY MOROSO y su } \\
\text { mujer. } \\
\text { 1538: ALONSO GÓMEZ }\end{array}$ & $\begin{array}{l}1 \text { HUERTO y VIÑA en la } \\
\text { Varga de Turieno } \\
1 \text { CUARTO DE LINAZA } \\
4 \text {-5 CÁNTARAS DE VINO } \\
1 \text { TIERRA en la Vega de } \\
\text { Turieno } \\
1 \text { SESTERO DE TRIGO } \\
1 \text { VIÑA en Llan de Viñas } \\
4 \text { CÁNTARAS DE VINO } \\
1 \text { VIÑA en Llan de Viñas } \\
3 \text { CÁNTARAS DE VINO } \\
1 \text { PRADO en Avenavas } \\
3 \text { CARGAS DE YERBA } \\
1 \text { PRADO en el prado de la } \\
\text { Serna } \\
1 \text { MOSTELA DE YERBA } \\
1 \text { HAZA DE PRADO en la } \\
\text { Serna } \\
1 \text { CARGA DE YERBA } \\
1 \text { VIÑA en la fuente de } \\
\text { Tabejana }\end{array}$ & 1 MIEDRO DE VINO & $\begin{array}{l}\text { 4-5 MIEDROS DE VINO } \\
4 \text { CÁNTARAS DE VINO } \\
1 \text { CARGA DE YERBA }\end{array}$ & $\begin{array}{l}\text { 1499 y 1515: PAGA DE } \\
\text { INFURCIÓN } 2 \text { FANEGAS } \\
\text { DE TRIGO Y } 2 \text { 1⁄2 } \\
\text { CÁNTARAS DE VINO. } \\
\text { 1538: } 2 \text { FANEGAS DE } \\
\text { TRIGO Y } 3 \text { CÁNTARAS } \\
\text { DE VINO. }\end{array}$ \\
\hline
\end{tabular}




\begin{tabular}{|c|c|c|c|c|}
\hline & $\begin{array}{l}1 \text { CÁNTARA DE VINO } \\
1 \text { VIÑA en la Pasadilla del } \\
\text { prado de Mieses } \\
2 \text { MIEDROS DE VINO } \\
\text { NO FIGURA }\end{array}$ & $\begin{array}{l}2 \text { CÁNTARAS DE VINO } \\
3 \text { MIEDROS DE VINO } \\
\text { NO FIGURA }\end{array}$ & $\begin{array}{l}1 \text { VIÑA en la valleja de } \\
\text { Viña Mayor } \\
3 \text { MIEDROS DE VINO }\end{array}$ & \\
\hline $\begin{array}{l}\text { 1499: Solar de Alonso } \\
\text { Gómez } \\
\text { 1515: Solar de Juana la } \\
\text { sorda } \\
\text { 1499: ALONSO GÓMEZ } \\
\text { DE MIESES } \\
\text { 1515: JUANA LA } \\
\text { SORDA } \\
\text { 1538: HEREDEROS DE } \\
\text { DIEGO DE BARÓ }\end{array}$ & $\begin{array}{l}1 \text { HUERTO en Mieses (que } \\
\text { solía ser solar) } \\
1 \text { 12 HEMINA DE LINAZA } \\
1 \text { TIERRA en el prado de } \\
\text { Mieses } \\
1 \text { SESTERO DE TRIGO } \\
1 \text { VIÑA en la serna Pumar } \\
1 \text { CÁNTARA DE VINO } \\
1 \text { VIÑA en Valladar } \\
1 \text { CÁNTARA DE VINO } \\
1 \text { VIÑA en Viña Mayor } \\
2 \text { MIEDROS DE VINO } \\
1 \text { VIÑA en Canpijon } \\
1 \text { CÁNTARA DE VINO }\end{array}$ & $\begin{array}{l}1 \text { CUARTO DE TRIGO } \\
4 \text { CÁNTARAS DE VINO } \\
1 \text { VIÑA y TIERRA en } \\
\text { Canpijón } \\
4 \text { CÁNTARAS DE VINO } \\
3 \text { HEMINAS DE TRIGO }\end{array}$ & 4 CÁNTARAS DE VINO & $\begin{array}{l}\text { PAGA DE INFURCIÓN } 7 \\
\text { HEMINAS DE TRIGO Y } \\
10 \text { AZUMBRES DE VINO }\end{array}$ \\
\hline
\end{tabular}




\begin{tabular}{|c|c|c|c|c|}
\hline & $\begin{array}{l}\text { HAZA DE VIÑA en la cuesta } \\
\text { de Tabejana } \\
1 \text { MIEDRO DE VINO } \\
1 \text { VIÑA al Valladar } \\
\dot{¿} ?\end{array}$ & $\begin{array}{l}2 \text { MIEDROS DE VINO } \\
1 \text { PANEDO al Valladar } \\
3 \text { MIEDROS DE VINO }\end{array}$ & NO FIGURA & \\
\hline $\begin{array}{l}\text { Solar del curero. } \\
\text { 1538: Al presente está } \\
\text { derrocado } \\
\text { 1499: JUAN GÓMEZ DE } \\
\text { MOGROVEJO, CURA } \\
\text { 1515: Herederos de } \\
\text { JUAN GÓMEZ DE } \\
\text { MOGROVEJO } \\
\text { 1538: ANTONIO LASO } \\
\text { DE MOGROVEJO. }\end{array}$ & $\begin{array}{l}1 \text { HUERTO junto al solar } \\
1 \text { HEMINA DE LINAZA } \\
1 \text { VIÑA en Viña Mayor } \\
2 \text { CÁNTARAS DE VINO } \\
1 \text { VIÑA so la Orden de San } \\
\text { Lázaro } \\
2 \text { CÁNTARAS DE VINO } \\
1 \text { PRADO en el Habar } \\
1 \text { CARRO DE YERBA } \\
1 \text { HAZA DE VIÑA en la } \\
\text { Molina } \\
2 \text { CÁNTARAS DE VINO }\end{array}$ & 3 CÁNTARAS DE VINO & $\begin{array}{l}2 \text { CÁNTARAS DE VINO } \\
1 \text { HAZA DE TIERRA en } \\
\text { la Molina } \\
1 \text { HEMINA DE TRIGO }\end{array}$ & $\begin{array}{l}\text { PAGA DE INFURCION } 5 \\
\text { HEMINAS DE TRIGO Y } 7 \\
\text { AZUMBRES DE VINO }\end{array}$ \\
\hline $\begin{array}{l}\text { Solar de Hondón de villa } \\
\text { 1499: JUAN GÓMEZ DE } \\
\text { MOGROVEJO, CURA } \\
\text { 1515: Herederos de } \\
\text { JUAN GÓMEZ DE } \\
\text { MOGROVEJO } \\
\text { 1538: ANTONIO LASO }\end{array}$ & $\begin{array}{l}1 \text { VIÑA en Viña Mayor } \\
2 \text { MIEDROS DE VINO } \\
1 \text { VIÑA en Viña Mayor } \\
1 \text { MIEDRO DE VINO } \\
1 \text { VIÑA en Olinas } \\
2 \text { MIEDROS DE VINO }\end{array}$ & 3 MIEDROS DE VINO & & $\begin{array}{l}\text { PAGA DE INFURCION } 10 \\
\text { HEMINAS DE TRIGO Y } \\
14 \text { AZUMBRES DE VINO. } \\
\text { 1538: Se agrega: “De } \\
\text { manera que paga por los } \\
\text { dichos solares y préstamos } \\
\text { 15 HEMINAS DE TRIGO } \\
\text { Y } 21 \text { AZUMBRES DE } \\
\text { VINO” }\end{array}$ \\
\hline
\end{tabular}




\begin{tabular}{|l|l|l|l|l|}
\hline DE MOGROVEJO. & $\begin{array}{l}1 \text { TIERRA en el Pumar de } \\
\text { Mieses } \\
\text { 1 CUARTO DE TRIGO }\end{array}$ & $\begin{array}{l}\text { Tumar Y VIÑA en el } \\
\text { 1 MIEDRO DE VINO } \\
\text { 3 HEMINAS DE TRIGO }\end{array}$ & $\begin{array}{l}\text { 1 MIEDRO DE VINO } \\
\text { 1 SESTERO DE TRIGO }\end{array}$ & \\
& & & \\
\hline
\end{tabular}

SOLARES EN CONGARNA

\begin{tabular}{|c|c|c|c|c|}
\hline TENENTE & 1499 & 1515 & 1538 & RENTA \\
\hline $\begin{array}{l}\text { 1499: ALONSO DE } \\
\text { FLORANES } \\
\text { 1515: HIJOS DE } \\
\text { ALONSO DE } \\
\text { FLORANES } \\
\text { 1538: TORIBIO DE } \\
\text { CONGARNA, merino, y } \\
\text { JUAN GÓMEZ DE } \\
\text { CONGARNA ( su } \\
\text { sobrino) }\end{array}$ & $\begin{array}{l}\text { LAS SERNAS DE SAN } \\
\text { JULIAN DE CONGARNA, } \\
\text { CON SU PRADO que está } \\
\text { adentro. } \\
8 \text { FANEGAS DE TRIGO } \\
\text { 5-6 CARROS DE YERBA } \\
1 \text { TIERRA en la Encyerta } \\
1 \text { SESTERO DE TRIGO }\end{array}$ & & $\begin{array}{l}\text { 10-12 FANEGAS TRIGO } \\
\text { 4-5 CARROS DE YERBA }\end{array}$ & $\begin{array}{l}\text { PAGA DE INFURCIÓN } \\
2 \text { FANEGAS DE TRIGO }\end{array}$ \\
\hline $\begin{array}{l}\text { 1499: NO FIGURA } \\
\text { 1515: HIJOS DE } \\
\text { ALONSO DE } \\
\text { FLORANES } \\
\text { 1538: PEDRO } \\
\text { GONÇALEZ DE } \\
\text { CONGARNA Y MAYOR } \\
\text { (mujer de HERNANDO } \\
\text { DE PRÍO) }\end{array}$ & & $\begin{array}{l}1 \text { HUERTO } \\
\dot{¿} ?\end{array}$ & & $\begin{array}{l}\text { PAGA DE INFURCIÓN } \\
3 \text { PANES Y } 1 \text { GALLINA. }\end{array}$ \\
\hline
\end{tabular}




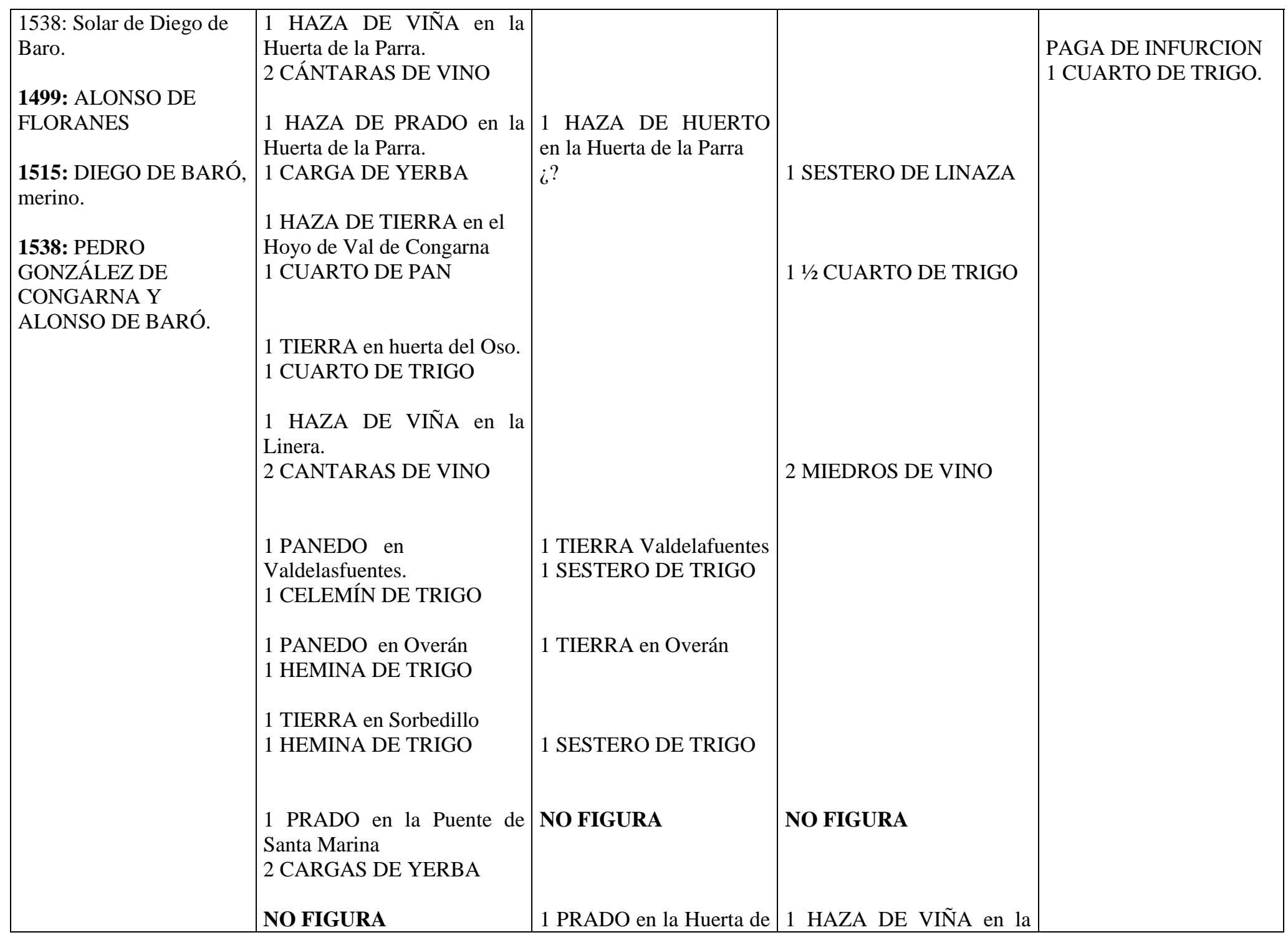




\begin{tabular}{|c|c|c|c|}
\hline & & $\begin{array}{l}\text { la Parra } \\
1 \text { CARGA DE YERBA }\end{array}$ & $\begin{array}{l}\text { Huerta de la Parra } \\
1 \text { CÁNTARA DE VINO }\end{array}$ \\
\hline $\begin{array}{l}\text { 1499 y 1515: Solar de } \\
\text { JUAN DE DIEGO } \\
\text { 1538: Solar de MARTÍN } \\
\text { DE JUAN DE DIEGO. } \\
\text { 1499: HIJOS DE JUAN } \\
\text { DE DIEGO } \\
\text { 1515: PEDRO LEBEÑA } \\
\text { 1538: MARTÍN DE } \\
\text { JUAN DE DIEGO, } \\
\text { JUAN (su hijo), HIJOS } \\
\text { DE PEDRO LEBEÑA Y } \\
\text { LA MUJER DEL ROJO. }\end{array}$ & $\begin{array}{l}1 \text { VIÑA en el llano de Mus } \\
2 \text { CÁNTARAS DE VINO } \\
1 \text { VIÑA en la Linera } \\
2 \text { CÁNTARAS DE VINO } \\
1 \text { VIÑA en Rebollín } \\
3 \text { CÁNTARAS DE VINO } \\
1 \text { PANEDO en la Pandella } \\
1 \text { HEMINA DE PAN } \\
1 \text { VIÑA en el Posadorio } \\
1 \text { CÁNTARA DE VINO } \\
1 \text { VIÑA en la Huerta la Parra } \\
4 \text { CÁNTARAS DE VINO } \\
1 \text { VIÑA en so Congarna } \\
2 \text { MIEDROS DE VINO } \\
1 \text { VIÑA en so Congarna } \\
10 \text { CÁNTARAS DE VINO } \\
1 \text { TIERRA entre lintes } \\
1 \text { SESTERO DE TRIGO } \\
1 \text { VIÑA Y TIERRA en la } \\
\text { vega de So Congarna } \\
3 \text { MIEDROS DE VINO } \\
1 \text { SESTERO DE PAN }\end{array}$ & $\begin{array}{l}1 \text { MIEDRO DE VINO } \\
1 \text { MIEDRO DE VINO } \\
4 \text { CÁNTARAS DE VINO } \\
1 \text { TIERRA en la Pandella } \\
2 \text { CÁNTARAS DE VINO } \\
1 \text { MIEDRO DE VINO } \\
6 \text { MIEDROS DE VINO } \\
3 \text { HEMINAS DE PAN }\end{array}$ & $\begin{array}{l}\dot{¿} \text { ? } \\
2 \text { MIEDROS DE VINO } \\
1 \text { VIÑA a la Fuente de Mus } \\
2 \text { MIEDROS DE VINO }\end{array}$ \\
\hline
\end{tabular}




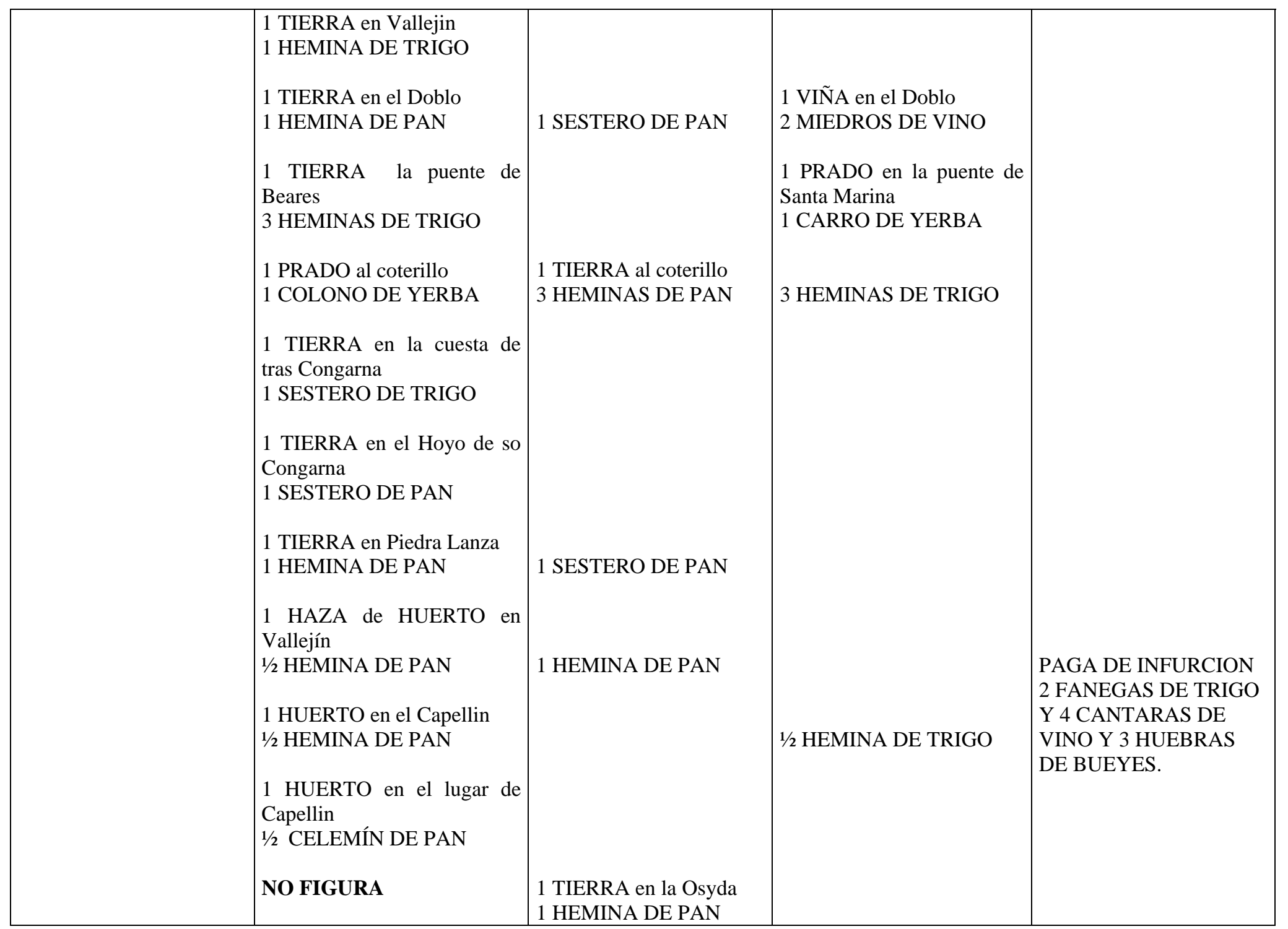




\begin{tabular}{|c|c|c|c|c|}
\hline & $\begin{array}{l}\text { NO FIGURA } \\
1 \text { PEDAZO cerca de la casa } \\
\text { de Pedro Martínez } \\
1 \text { \&2 CELEMIN DE PAN } \\
1 \text { PANEDO en } \\
\text { Valdelasfuentes } \\
1 \text { SESTERO DE TRIGO } \\
1 \text { TIERRA al sorbal de } \\
\text { hondón de las Fuentes } \\
1 / 2 \text { HEMINA DE PAN } \\
1 \text { TIERRA en huerta del Oso } \\
1 \text { HEMINA DE PAN }\end{array}$ & $\begin{array}{l}1 \text { TIERRA en hondon de } \\
\text { la Encyerta } \\
1 \text { HEMINA DE PAN } \\
\dot{c} \text { ? } \\
1 \text { TIERRA en } \\
\text { Valdelasfuentes } \\
1 \text { SESTERO DE PAN } \\
1 \text { TIERRA Y VIÑA en } \\
\text { huerta del Oso } \\
1 \text { SESTERO DE PAN } \\
1 \text { MIEDRO DE VINO }\end{array}$ & $\begin{array}{l}1 \text { HEMINA DE TRIGO } \\
\text { NO FIGURA } \\
1 \text { VIÑA en huerta del Oso } \\
3 \text { MIEDROS DE VINO }\end{array}$ & \\
\hline $\begin{array}{l}\text { 1499: Solar de HIJOS DE } \\
\text { PEDRO MARTÍNEZ } \\
\text { 1515: Solar de PEDRO } \\
\text { MARTÍNEZ } \\
\text { 1538: Solar de ALONSO } \\
\text { DEL ALMIRANTE } \\
\text { 1499: ELVIRA e HIJOS } \\
\text { DE PEDRO MARTÍNEZ } \\
\text { 1515: Lo llevaba Pedro } \\
\text { Martínez y ahora lo lleva } \\
\text { DIEGO DE BARÓ y } \\
\text { TORIBIO DEL }\end{array}$ & $\begin{array}{l}1 \text { ERA que está sobre el solar } \\
1 \text { VIÑA en el llano de Mus } \\
\text { 3-4 MIEDROS DE VINO } \\
1 \text { TIERRA en la vega de } \\
\text { Turieno } \\
1 \text { CUARTO DE TRIGO }\end{array}$ & 5-6 MIEDROS DE VINO & $\begin{array}{l}\text { 3-4 MIEDROS DE VINO } \\
\text { NO FIGURA }\end{array}$ & $\begin{array}{l}\text { PAGA DE INFURCIÓN } \\
14 \text { AZUMBRES DE } \\
\text { VINO }\end{array}$ \\
\hline
\end{tabular}




\begin{tabular}{|c|c|c|c|c|}
\hline $\begin{array}{l}\text { ALMIRANTE } \\
\text { 1538: ALONSO DEL } \\
\text { ALMIRANTE y PEDRO } \\
\text { GONZÁLEZ DE } \\
\text { CONGARNA }\end{array}$ & & & & \\
\hline $\begin{array}{l}\text { Solar de TORIBIO DEL } \\
\text { BARRIAL } \\
\text { 1499 y 1515: HIJOS DE } \\
\text { TORIBIO DEL } \\
\text { BARRIAL } \\
\text { 1538: TORIBIO DEL } \\
\text { BARRIAL Y ALONSO } \\
\text { DE ANTÓN }\end{array}$ & 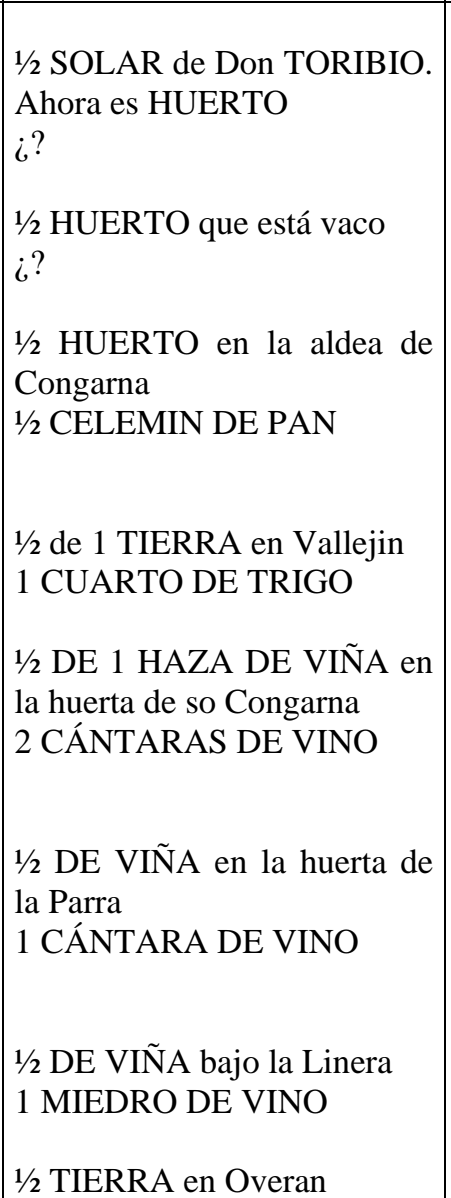 & $\begin{array}{l}1 / 2 \text { de } 1 \text { VIÑA [que solía } \\
\text { ser huerto] } \\
3 \text { CANTARAS DE VINO }\end{array}$ & $\begin{array}{l}\text { 1/2 HUERTO en la aldea de } \\
\text { Congarna } \\
1 \frac{2}{2} \text { CELEMIN DE PAN } \\
\text { NO FIGURA } \\
\text { 2-3 CÁNTARAS DE VINO } \\
1 \text { CÁNTARA DE VINO }\end{array}$ & $\begin{array}{l}\text { PAGA DE INFURCION } \\
1 \text { CUARTO DE TRIGO }\end{array}$ \\
\hline
\end{tabular}




\begin{tabular}{|c|c|c|c|c|}
\hline & $\begin{array}{l}1 \text { 1⁄2 HEMINA DE TRIGO } \\
1 / 2 \text { VIÑA en hondon de } \\
\text { Valdelasfuentes } \\
1 \text { CANTARA DE VINO } \\
1 \text { TIERRA en la Pandilla } \\
\text { (solía ser viña) } \\
1 \text { SESTERO CENTENO } \\
1 / 2 \text { HUERTO en Vallejin } \\
\text { ¿? } \\
1 \text { HAZA DE VIÑA en la } \\
\text { Valleja } \\
2 \text { CANTARAS DE VINO } \\
1 \text { TIERRA en la Encyerta } \\
1 / 2 \text { HEMINA DE PAN }\end{array}$ & 1 SESTERO DE LINAZA & \begin{tabular}{|l|}
1 SESTERO DE TRIGO \\
$1 / 2$ TIERRA en hondon de \\
Overán \\
1 HEMINA DE PAN \\
1 SESTERO DE TRIGO \\
$1 / 2$ HEMINA DE PAN
\end{tabular} & \\
\hline $\begin{array}{l}\text { 1499 y 1515: Solar de } \\
\text { TORIBIO EL } \\
\text { ALMIRANTE } \\
\text { 1538: Solar de ALONSO } \\
\text { DEL ALMIRANTE } \\
\text { 1499 y 1515: TORIBIO } \\
\text { EL ALMIRANTE } \\
\text { 1538: ALONSO DEL } \\
\text { ALMIRANTE }\end{array}$ & $\begin{array}{l}1 / 2 \text { HUERTO junto al solar } \\
1 \text { HEMINA DE TRIGO } \\
1 / 2 \text { HUERTO en Congarna } \\
1 / 2 \text { CELEMIN DE TRIGO } \\
\text { 1/2 HUERTO en Vallejin } \\
1 \text { CELEMIN de LINAZA } \\
1 \text { He HAZA DE VIÑA en la } \\
\text { huerta de so Congarna } \\
2 \text { CÁNTARAS DE VINO }\end{array}$ & $\begin{array}{l}1 \text { SESTERO DE LINAZA } \\
3 \text { CÁNTARAS DE VINO }\end{array}$ & $\begin{array}{l}1 \text { HEMINA de LINAZA } \\
1 \text { MIEDRO DE VINO }\end{array}$ & $\begin{array}{l}\text { PAGA DE INFURCION } \\
1 \text { CUARTO DE TRIGO }\end{array}$ \\
\hline
\end{tabular}




\begin{tabular}{|c|c|c|c|c|}
\hline & $\begin{array}{l}1 \text { HAZA DE VIÑA huerta la } \\
\text { Parra } \\
1 \text { CÁNTARA DE VINO } \\
\text { 1⁄2 VIÑA en la Linera } \\
2 \text { MIEDROS DE VINO } \\
\text { 1⁄2 VIÑA en Overan } \\
2 \text { CÁNTARAS DE VINO } \\
1 \text { TIERRA en hondon de } \\
\text { Valdelasfuentes } \\
\text { 1/2 HEMINA DE PAN } \\
1 \text { TIERRA en la vega de so } \\
\text { Congarna } \\
3 \text { HEMINAS DE TRIGO } \\
\text { NO FIGURA }\end{array}$ & $\begin{array}{l}1 \text { MIEDRO DE VINO } \\
\text { 1/2 VIÑA en hondon de la } \\
\text { riega de Valdelasfuentes } \\
2 \text { CÁNTARAS DE VINO } \\
\text { NO FIGURA }\end{array}$ & $\begin{array}{l}1 \text { VIÑA en so Congarna } \\
1 \text { MIEDRO DE VINO } \\
1 \text { TIERRA en Overán } \\
1 \text { SESTERO DE TRIGO } \\
1 \text { TIERRA en hondon de la } \\
\text { riega de Valdelasfuentes } \\
1 \text { HEMINA DE TRIGO } \\
1 \text { HAZA DE TIERRA en la } \\
\text { Encierta } \\
\text { ¿? }\end{array}$ & \\
\hline \begin{tabular}{|l|} 
Solar del OSO \\
1499: TORIBIO EL \\
ALMIRANTE, TORIBIO \\
DE JUANA y GARCÍA \\
DE LA FRECHA \\
\\
1515 y 1538: TORIBIO \\
EL ALMIRANTE y \\
OTROS \\
\end{tabular} & $\begin{array}{l}1 \text { HUERTO } \\
1 \text { HEMINA DE PAN } \\
1 \text { VIÑA sobre la serna de } \\
\text { Mus } \\
\dot{¿} ? \\
\text { NO FIGURA }\end{array}$ & $\begin{array}{l}\text { 5-6 MIEDROS DE VINO } \\
1 \text { VIÑA en huerta del Oso } \\
6 \text { MIEDROS DE VINO }\end{array}$ & $\begin{array}{l}\text { NO FIGURA } \\
1 \text { MIEDRO DE VINO }\end{array}$ & $\begin{array}{l}\text { PAGA DE INFURCION } \\
5 \text { HEMINAS DE TRIGO } \\
\text { Y } 7 \text { AÇUNBRES DE } \\
\text { VINO EN CADA AÑO }\end{array}$ \\
\hline
\end{tabular}




\begin{tabular}{|c|c|c|c|c|}
\hline $\begin{array}{l}\text { 1499: Solar de TORIBIO } \\
\text { DE JUANA (lo lleva T de } \\
\text { Juana) } \\
\text { 1515: Solar de MARÍA } \\
\text { PEDRO y de sus hijos (lo } \\
\text { lleva María Pedro y sus } \\
\text { hijos) } \\
\text { APEO 1538: NO } \\
\text { FIGURA }\end{array}$ & $\begin{array}{l}1 \text { TIERRA y PRADO encima } \\
\text { de la Serna de Mus } \\
1 \text { 1⁄2 FANEGAS DE TRIGO } \\
\text { 3-4 CARGAS DE YERBA }\end{array}$ & [blanco] & & $\begin{array}{l}\text { 1499: PAGA DE } \\
\text { INFURCIÓN } 6 \\
\text { HEMINAS DE TRIGO }\end{array}$ \\
\hline $\begin{array}{l}\text { Solar DE LA CANAL } \\
\text { 1499: TORIBIO DE } \\
\text { JUANA y DIEGO DE } \\
\text { BARÓ } \\
\text { 1515: MARÍA PEDRO } \\
\text { con sus hijos y DIEGO } \\
\text { DE BARÓ } \\
\text { 1538: PEDRO DE PRÍO } \\
\text { y DIEGO DE BARÓ }\end{array}$ & $\begin{array}{l}\text { El HUERTO del Polvo } \\
1 \text { 1⁄2 HEMINA DE TRIGO } \\
1 \text { HUERTO junto con el solar } \\
1 / 2 \text { CELEMIN DE TRIGO }\end{array}$ & 3 HEMINAS de LINAZA & 1 1⁄2 HEMINA DE TRIGO & $\begin{array}{l}\text { PAGA LA MITAD DEL } \\
\text { SOLAR Y PRESTAMOS } \\
1 \text { SESTERO DE TRIGO } \\
\text { (Tachado } 1 / 2 \text { cuarto) } \\
\text { 1499 y 1538: PAGA DE } \\
\text { INFURCION } 1 \\
\text { SESTERO DE TRIGO Y } \\
1 / 2 \text { CANTARA DE VINO. }\end{array}$ \\
\hline $\begin{array}{l}\text { LA PARTE DE DIEGO } \\
\text { DE BARÓ }\end{array}$ & $\begin{array}{l}1 \text { PRADO en Otero } \\
\text { 2-3 CARGAS de YERBA } \\
1 \quad \text { HAZA DE VIÑA en } \\
\text { Camares } \\
\text { 2-4 CÁNTARA de VINO } \\
\text { 1 VIÑA en Camares } \\
\text { 2-3 CANTARA de VINO }\end{array}$ & $\begin{array}{l}1 \text { HUERTO } \\
6 \text { HEMINAS de LINAZA } \\
4 \text { CÁNTARAS DE VINO } \\
2 \text { CÁNTARAS DE VINO }\end{array}$ & 1 SESTERO DE LINAZA & $\begin{array}{l}\text { PAGA DE INFURCION } \\
1 \text { SESTERO DE TRIGO, } \\
1 / 2 \text { CANTARA DE VINO } \\
\text { Y } 1 \text { GALLINA }\end{array}$ \\
\hline
\end{tabular}




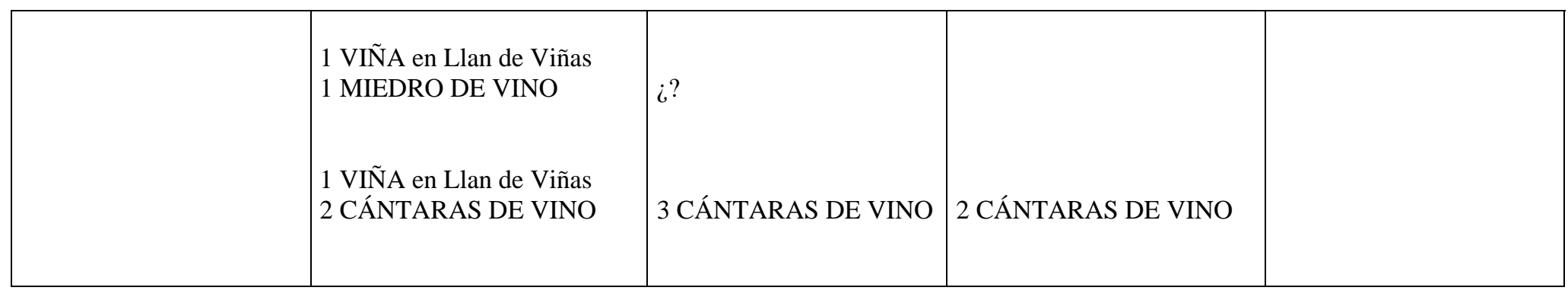

SOLARES EN TURIENO

\begin{tabular}{|c|c|c|c|c|}
\hline TENENTE & 1499 & 1515 & 1538 & RENTA \\
\hline $\begin{array}{l}\begin{array}{l}\text { Solar de Era (es con su } \\
\text { corral) }\end{array} \\
\text { 1499: DIEGO DE } \\
\text { FLORANES } \\
\text { 1515: DIEGO } \\
\text { FERRANDEZ DE } \\
\text { FLORANES CURA DE } \\
\text { COLLO } \\
\text { 1538: PEDRO HERREN } \\
\text { y MARIA HERNANDEZ } \\
\text { SU MUGER }\end{array}$ & $\begin{array}{l}1 \text { HAZA DE VIÑA en la } \\
\text { Pereda } \\
4 \text { CÁNTARAS DE VINO } \\
1 \text { HAZA DE TIERRA en la } \\
\text { vega de Torieno } \\
1 \text { CELEMÍN DE TRIGO } \\
1 \text { VIÑA en Llan de Viñas } \\
1 \text { CÁNTARA DE VINO } \\
1 \text { HAZA DE HUERTO en la } \\
\text { Porraliega } \\
1 \text { HEMINA DE LINAZA } \\
1 \text { HAZA DE HUERTO en la } \\
\text { Calleja } \\
1 \text { HEMINA DE LINAZA }\end{array}$ & $\begin{array}{l}1 \text { MIEDRO DE VINO } \\
1 \text { HAZA DE VIÑA en la } \\
\text { Vega de Torieno } \\
3 \text { CÁNTARAS DE VINO } \\
2 \text { MIEDROS DE VINO } \\
\text { (estando bien labrada) }\end{array}$ & $\begin{array}{l}\text { 1/2 HEMINA DE LINAZA } \\
1 \text { HEMINA DE LINAZA }\end{array}$ & $\begin{array}{l}\text { 1499: PAGA DE } \\
\text { INFURCIÓN } \\
\text { 1 SESTERO DE TRIGO, } \\
4 \text { AZUMBRES DE VINO } \\
\text { Y 1 HUEBRA DE } \\
\text { BUEYES. } \\
\text { 1515 y 1538: PAGA DE } \\
\text { INFURCION } 1 \text { CUARTO } \\
\text { DE TRIGO, } 1 \\
\text { CANTARA DE VINO Y } \\
\text { 1 HUEBRA DE } \\
\text { BUEYES. HA DE } \\
\text { QUEDAR EN UN } \\
\text { HEREDERO Y NO } \\
\text { MAS. } \\
\text { Agregado 1515: DIOSE }\end{array}$ \\
\hline
\end{tabular}




\begin{tabular}{|c|c|c|c|c|}
\hline & $\begin{array}{l}1 \text { HAZA DE HUERTO en la } \\
\text { Calleja } \\
1 \text { CUARTO DE LINAZA } \\
1 \text { VIÑA en so Casa } \\
4 \text { CÁNTARAS DE VINO }\end{array}$ & & NO FIGURA & $\begin{array}{l}\text { A JUAN DE BARÓ Y } \\
\text { PAGA } 3 \text { CUARTOS DE } \\
\text { TRIGO Y } 2 \text { HUEBRAS }\end{array}$ \\
\hline $\begin{array}{l}\text { Otro solar en el corral de } \\
\text { Era } \\
\text { 1499 Y 1515: JUAN DE } \\
\text { DIEGO } \\
\text { 1538: MARTIN DE } \\
\text { BARÓ }\end{array}$ & & & & $\begin{array}{l}\text { SOLÍA PAGAR LOS } \\
\text { MEDIOS AÑOS } 3 \\
\text { PANES Y UNA } \\
\text { GALLINA. PAGA EN } \\
\text { CADA AÑO } 1 \\
\text { GALLINA Y NO MAS. }\end{array}$ \\
\hline $\begin{array}{l}\text { Otro solar de Era } \\
\text { 1499: NO FIGURA } \\
\text { 1515 y 1538: GARCÍA } \\
\text { DE NORIEGA (Fue de } \\
\text { García Ponce) }\end{array}$ & & $\begin{array}{l}1 \text { VIÑA en Olinas } \\
1 \text { MIEDRO DE VINO } \\
1 \text { VIÑA en Viña Mayor } \\
1 \text { MIEDRO DE VINO }\end{array}$ & & $\begin{array}{l}\text { 1515 y 1538: PAGA DE } \\
\text { INFURCION } 5 \\
\text { HEMINAS DE TRIGO, } \\
7 \text { AZUMBRES DE VINO } \\
\text { Y } 11 / 2 \text { HUEBRAS DE } \\
\text { BUEYES }\end{array}$ \\
\hline $\begin{array}{l}\text { Otro solar de Era } \\
\text { 1499: GARCÍA Y } \\
\text { PEDRO, hijos de } \\
\text { GONZALO PONCE Y } \\
\text { OTROS } \\
\text { 1515: GARCÍA PONCE, } \\
\text { EL MOZO Y OTROS. }\end{array}$ & $\begin{array}{l}1 \text { VIÑA Y TIERRA en la } \\
\text { puente de Turieno } \\
5 \text { MIEDROS DE VINO } \\
6 \text { HEMINAS DE TRIGO } \\
1 \text { VIÑA en Tabejana } \\
2 \text { MIEDROS DE VINO } \\
1 \text { VIÑA en la Pereda } \\
\end{array}$ & $\begin{array}{l}12 \text { MIEDROS DE VINO } \\
3 \text { HEMINAS DE TRIGO } \\
3 \text { MIEDROS DE VINO }\end{array}$ & $\begin{array}{l}1 \text { VIÑA } \\
\dot{¿} ? \\
2 \text { MIEDROS DE VINO }\end{array}$ & $\begin{array}{l}\text { PAGA EL DICHO } \\
\text { SOLAR Y PRESTAMOS } \\
10 \text { HEMINAS DE } \\
\text { TRIGO } 14 \text { AZUMBRES } \\
\text { DE VINO Y } 3 \\
\text { HUEBRAS DE } \\
\text { BUEYES }^{1} .\end{array}$ \\
\hline
\end{tabular}

${ }^{1}$ APEO 1538: SACANDO LO QUE LLEVAN LOS HEREDEROS DE DIEGO DE FLORANES. Queda que HAN DE PAGAR 9 HEMINAS DE TRIGO, 13 AZUMBRES DE VINO Y $2 \frac{1 / 2}{2}$ HUEBRAS DE BUEYES. HA DE PAGAR GARCI PONCE 5 HEMINAS DE TRIGO, 7 AZUMBRES DE VINO Y 1 1⁄2 HUEBRAS DE BUEYES. 


\begin{tabular}{|c|c|c|c|}
\hline & 6 MIEDROS DE VINO & & \\
\hline $\begin{array}{l}\text { 1499: Solar de Gonzalo } \\
\text { Sánchez. } \\
\text { 1515: Solar en el corral de } \\
\text { Era } \\
\text { 1538: NO FIGURA } \\
\text { 1499: GONZALO } \\
\text { SÁNCHEZ } \\
\text { 1515: JUAN SÁNCHEZ }\end{array}$ & $\begin{array}{l}1 \text { VIÑA en Olinas } \\
3 \text { CANTARAS DE VINO } \\
1 \text { TIERRA en la puente de } \\
\text { Periguales } \\
1 \text { CUARTO DE TRIGO } \\
\text { NO FIGURA } \\
\\
1 \text { VIÑA en Viña Mayor } \\
1 \text { MIEDRO DE VINO } \\
1 \text { PRADO Y TIERRA sobre } \\
\text { Viña Mayor } \\
1 \text { SESTERO DE TRIGO } \\
1 \text { CARGA DE YERBA } \\
1 \text { TIERRA en Valdebalino } \\
1 \text { HEMINA DE PAN }\end{array}$ & $\begin{array}{l}1 \text { HAZA DE VIÑA en la } \\
\text { Molina } \\
\dot{¿} ? \\
2 \text { MIEDROS DE VINO }\end{array}$ & $\begin{array}{l}\text { 1499 Y 1515: PAGA DE } \\
\text { INFURCION } 1 \\
\text { SESTERO DE TRIGO Y } \\
\text { 1/2 CANTARA DE VINO. } \\
\text { Agregado 1515: LA } \\
\text { MITAD DE ESTE } \\
\text { SOLAR SE TROCÓ POR } \\
\text { OTRO EN FLORANES }\end{array}$ \\
\hline $\begin{array}{l}\text { 1499: ALONSO DEL } \\
\text { CALVO Y TORIBIO, su } \\
\text { heredero. } \\
\text { 1515: ALONSO EL } \\
\text { CALVO y sus sobrinos } \\
\\
\text { 1538: MARIA (nieta de } \\
\text { Alonso del Calvo) y } \\
\text { JUAN DE FLORANES }\end{array}$ & & & $\begin{array}{l}\text { PAGA DE INFURCION } \\
3 \text { PANES Y } 1 \text { GALLINA. }\end{array}$ \\
\hline
\end{tabular}




\begin{tabular}{|c|c|c|c|c|}
\hline $\begin{array}{l}\text { 1515: Solar de la Pelaya } \\
\text { 1538: Solar de Alonso de } \\
\text { Sotres. } \\
\text { 1499: PEDRO DE } \\
\text { OTERO } \\
\text { 1515: TORIBIO DE } \\
\text { PEDRO DE OTERO } \\
\text { 1538: JUAN DE OTERO }\end{array}$ & $\begin{array}{l}\text { NO FIGURA } \\
\text { NO FIGURA }\end{array}$ & $\begin{array}{l}1 \text { VIÑA en la Molina } \\
2 \text { CARGAS DE UVAS } \\
1 \text { VIÑA entrante la Mata } \\
\text { de Santiago } \\
3 \text { CARGAS DE UVAS }\end{array}$ & $\begin{array}{l}\dot{¿} \text { ? } \\
1 \text { VIÑA en la valleja del } \\
\text { cotero de la Mata } \\
\dot{¿} ?\end{array}$ & $\begin{array}{l}\text { 1499, } 1515 \text { y 1538: } \\
\text { PAGA DE INFURCION } \\
3 \text { PANES Y } 1 \text { GALLINA }\end{array}$ \\
\hline $\begin{array}{l}\text { 1499: Solar de Domingo } \\
\text { Ferrández } \\
\text { 1515: Solar de Juan de } \\
\text { Diego } \\
\text { 1499 y 1515: JUAN DE } \\
\text { DIEGO } \\
\text { 1538: MARTÍN DE } \\
\text { BARÓ y JUAN, su hijo }\end{array}$ & $\begin{array}{l}\text { HUERTO en la Huerta de } \\
\text { Santa María } \\
1 \text { CUARTO DE LINAZA } \\
1 \text { VIÑA en la puente de } \\
\text { Turieno } \\
2 \text { MIEDROS DE VINO } \\
1 \text { VIÑA en Mus, en los } \\
\text { Parrales } \\
4 \text { MIEDROS DE VINO } \\
1 \text { TIERRA en el cuerano } \\
1 \text { SESTERO DE TRIGO } \\
1 \text { VIÑA en los Barriales } \\
2 \text { CANTARAS DE VINO } \\
\text { NO FIGURA }\end{array}$ & $\begin{array}{l}1 \text { FANEGA DE LINAZA } \\
\\
1 \text { MIEDRO DE VINO } \\
1 \text { PRADO en Habar } \\
\text { (término de Argüébanes) } \\
1 \quad 1 / 2 \quad \text { CARROS DE } \\
\text { YERBA }\end{array}$ & 1 CARRO DE YERBA & $\begin{array}{l}\text { PAGA DE INFURCION } \\
7 \text { HEMINAS DE TRIGO } \\
\text { Y } 2 \text { CÁNTARAS DE } \\
\text { VINO. HA DE ESTAR } \\
\text { EN } 1 \text { HEREDERO Y NO } \\
\text { MAS. } \\
\text { APEO 1538: IDEM, más } \\
1 \text { GALLINA “...que el } \\
\text { dicho solar de antes } \\
\text { pagava...”. }\end{array}$ \\
\hline $\begin{array}{l}\text { Solar de Garçia } \\
\text { 1499: HEREDEROS DE }\end{array}$ & $\begin{array}{l}1 \text { HUERTO y ERA junto a la } \\
\text { casa }\end{array}$ & NO FIGURA & NO FIGURA & \\
\hline
\end{tabular}




\begin{tabular}{|c|c|c|c|c|}
\hline $\begin{array}{l}\text { JUAN VALLEJO, } \\
\text { HEREDEROS DE } \\
\text { ALONSO DE LA } \\
\text { CALLEJA Y DE } \\
\text { ALONSO DE LA } \\
\text { FUENTE Y OTROS. } \\
\text { 1515: HEREDEROS DE } \\
\text { JUAN VALLEJO, } \\
\text { HEREDEROS DE } \\
\text { ALONSO DE LA } \\
\text { CALLEJA Y DEL } \\
\text { CLÉRIGO DE } \\
\text { ARGÜÉBANES } \\
\text { 1538: GÓMEZ DÍAZ Y } \\
\text { HERNANDO DE } \\
\text { MARTÍN }\end{array}$ & $\begin{array}{l}1 \text { CELEMÍN DE LINAZA } \\
1 \text { VIÑA en Mus en los } \\
\text { Parrales } \\
4-5 \text { MIEDROS DE VINO } \\
1 \text { VIÑA so la fuente de Mus } \\
8 \text { MIEDROS DE VINO } \\
\text { (siendo bien tratada) } \\
1 \text { VIÑA en el Arenal de Mus } \\
4 \text { MIEDROS DE VINO } \\
1 \text { VIÑA en esgovio de la } \\
\text { Cueva } \\
4 \text { CÁNTARAS DE VINO }\end{array}$ & 1 MIEDRO DE VINO & $\begin{array}{l}2 \text { MIEDROS DE VINO } \\
2 \text { MIEDROS DE VINO }\end{array}$ & $\begin{array}{l}\text { PAGA DE INFURCION } \\
10 \text { HEMINAS DE } \\
\text { TRIGO, } 14 \text { AZUMBRES } \\
\text { DE VINO Y } 3 \\
\text { HUEBRAS DE BUEYES. }\end{array}$ \\
\hline $\begin{array}{l}\text { 1499 y 1515: Solar de } \\
\text { Ferrán Ruiz } \\
\text { 1538: Solar de Elvira } \\
\text { Diez } \\
\text { 1499: ELVIRA DÍEZ DE } \\
\text { BARÓ } \\
\text { 1515: ALONSO GÓMEZ } \\
\text { DE BARÓ } \\
\text { 1538: PEDRO } \\
\text { GONZÁLEZ }\end{array}$ & $\begin{array}{l}1 \text { HAZA DE VIÑA sobre el } \\
\text { molino del monasterio } \\
2 \text { MIEDROS DE VINO } \\
1 \text { VIÑA sobre los molinos } \\
2 \text { MIEDROS DE VINO } \\
1 \text { VIÑA en la entrada de Llan } \\
\text { de Viñas } \\
2 \text { MIEDROS DE VINO }\end{array}$ & & $\begin{array}{l}4 \text { MIEDROS DE VINO } \\
4 \text { MIEDROS DE VINO }\end{array}$ & $\begin{array}{l}\text { PAGA DE INFURCION } \\
4 \text { CELEMINES DE } \\
\text { TRIGO Y } 51 \frac{1}{2} \\
\text { AZUMBRES DE VINO }\end{array}$ \\
\hline $\begin{array}{l}\text { TURIENO. Solar de } \\
\text { Pedro de Baró } \\
\text { 1499: La MUJER de }\end{array}$ & $\begin{array}{l}1 \text { VIÑA en la riega de la } \\
\text { Orden } \\
2 \text { MIEDROS DE VINO }\end{array}$ & & & \\
\hline
\end{tabular}




\begin{tabular}{|c|c|c|c|c|}
\hline $\begin{array}{l}\text { PEDRO DE BARÓ, } \\
\text { defunto. } \\
\text { 1515 y 1538: JUAN DE } \\
\text { BARÓ }\end{array}$ & $\begin{array}{l}1 \text { VIÑA en Tabejana (es en } \\
\text { Llan de Viñas) } \\
4 \text { CÁNTARAS DE VINO } \\
1 \text { HUERTO en la Huerta de } \\
\text { Santa Maria } \\
1 \text { SESTERO DE LINAZA } \\
1 \text { HUERTO en los Gallaranes } \\
1 \text { HEMINA DE LINAZA } \\
1 \text { VIÑA a la Piedra de Mus } \\
2 \text { CÁNTARAS DE VINO } \\
1 \text { VIÑA en la Piedra de Mus } \\
1 \text { MIEDRO DE VINO } \\
1 \text { PANEDO en Alzapierna } \\
1 \text { HEMINA DE PAN }\end{array}$ & $\begin{array}{l}\text { NO FIGURA } \\
1 \text { VIÑA en Alzapierna } \\
1 \text { MIEDRO DE VINO }\end{array}$ & NO FIGURA & $\begin{array}{l}\text { PAGA DE INFURCION } \\
1 \text { CUARTO DE TRIGO } \\
\text { Y 1 CÁNTARA DE } \\
\text { VINO. }\end{array}$ \\
\hline $\begin{array}{l}\text { Otro solar de La Calleja } \\
\text { 1499: ALONSO DE LA } \\
\text { CALLEJA } \\
\text { 1515:PEDRO } \\
\text { CEREBANES, TORIBIO } \\
\text { ESPINEL, ELVIRA } \\
\text { GÓMEZ, JUAN DE LA } \\
\text { FUENTE Y } \\
\text { HEREDEROS DE JUAN }\end{array}$ & $\begin{array}{l}1 \text { HUERTO que se dice de la } \\
\text { Calleja } \\
2 \text { FANEGAS DE LINAZA } \\
1 \text { HUERTO en la huerta de } \\
\text { Santa María } \\
\text { 5-6 HEMINA de LINAZA }\end{array}$ & $\begin{array}{l}3 \text { FANEGAS de LINAZA } \\
3 \text { CUARTOS de LINAZA }\end{array}$ & $\begin{array}{l}2 \text { FANEGAS DE LINAZA } \\
1 \text { CUARTO DE LINAZA }\end{array}$ & $\begin{array}{l}\text { PAGA DE INFURCION } \\
10 \text { HEMINAS DE } \\
\text { TRIGO, } 14 \text { AZUMBRES } \\
\text { DE VINO Y } 3 \\
\text { HUEBRAS DE } \\
\text { BUEYES }^{2} .\end{array}$ \\
\hline
\end{tabular}

${ }^{2}$ APEO 1538: DE ESTA DICHA INFURCION PAGAN LOS HEREDEROS DE DIEGO DE FLORANES 1 HEMINA DE TRIGO, 1 AZUMBRE DE VINO Y 1/2 HUEBRA DE BUEYES (lo cual pagan en el SOLAR DE ERA). DE MANERA QUE PAGA EL SOLAR DE LA CALLEJA Y PRÉSTAMOS 9 HEMINAS DE TRIGO, 13 AZUMBRES DE VINO Y 2 1/2 HUEBRAS DE BUEYES. 


\begin{tabular}{|c|c|c|c|c|}
\hline $\begin{array}{l}\text { DE VALLEJO } \\
\text { 1538: JUAN DE LA } \\
\text { FUENTE, EL MOZO y } \\
\text { PEDRO DE } \\
\text { CEREBANES }\end{array}$ & \begin{tabular}{|l}
1 VIÑA en la Cueva \\
2 MIEDROS DE VINO
\end{tabular} & 1 MIEDRO DE VINO & 2 CÁNTARAS DE VINO & \\
\hline $\begin{array}{l}\text { 1499 y 1515: Solar de } \\
\text { Gonçalo de la Calleja } \\
\text { 1538: Solar de Pedro de } \\
\text { Piasca } \\
\text { 1499: GONZALO DE LA } \\
\text { CALLEJA } \\
\text { 1515: JUAN DE LA } \\
\text { CALLEJA y JUANA, SU } \\
\text { HEREDERA } \\
\text { 1538: ALONSO DE } \\
\text { VALLEJO, yerno de } \\
\text { Pedro de Piasca. }\end{array}$ & $\begin{array}{l}1 \text { TIERRA en Santiago } \\
\dot{¿} \text { ? } \\
1 \text { PRADO en Barriberó, } \\
\text { donde dicen Fuentes } \\
3 \text { MOSTELAS DE YERBA } \\
1 \text { PRADO en Avenavas } \\
3 \text { CARGAS DE YERBA } \\
\text { 1VIÑA al portyllo de Mus } \\
1 \text { CARGA DE UVAS } \\
1 \text { VIÑA en la requexada de } \\
\text { Mus } \\
3 \text { CANTARAS DE VINO } \\
1 \text { PANEDO en la cuesta de } \\
\text { Mus } \\
\dot{¿} \text { ? }\end{array}$ & $\begin{array}{l}1 \text { VIÑA en la cuesta de } \\
\text { Mus } \\
\dot{¿} ?\end{array}$ & $\begin{array}{l}2 \text { CARGAS DE YERBA } \\
2 \text { CARGAS DE YERBA } \\
2 \text { CÁNTARAS DE VINO } \\
4 \text { CÁNTARAS DE VINO } \\
\text { 4 CÁNTARAS DE VINO }\end{array}$ & $\begin{array}{l}\text { PAGA DE INFURCION } \\
1 \text { CUARTO DE TRIGO }\end{array}$ \\
\hline
\end{tabular}




\begin{tabular}{|c|c|c|c|c|}
\hline $\begin{array}{l}\text { LA CALLEJA. Otro } \\
\text { solar de Gonzalo Vallejo } \\
\text { 1499 y 1515: GONZALO } \\
\text { VALLEJO } \\
\text { 1538: GUTTIERRE DE } \\
\text { LA CALLEJA }\end{array}$ & $\begin{array}{l}1 \text { VIÑA en Olinas } \\
4 \text { MIEDROS DE VINO } \\
1 \text { HUERTO en los huertos de } \\
\text { la Calleja } \\
1 \text { SESTERO DE LINAZA }\end{array}$ & & 3 HEMINAS DE LINAZA & $\begin{array}{l}\text { PAGA DE INFURCIÓN } \\
1 \text { FANEGA DE TRIGO }\end{array}$ \\
\hline $\begin{array}{l}\text { LA CALLEJA. Solar de } \\
\text { Antón Ruiz } \\
\text { 1499: JUAN } \\
\text { GONZALEZ DE } \\
\text { COLLAZOS Y GARCÍA, } \\
\text { su heredero } \\
\text { 1515: GARCÍA DE } \\
\text { MOLLEDA } \\
\text { 1538: JUAN DE } \\
\text { ERMIDA }\end{array}$ & $\begin{array}{l}1 \text { HUERTO sobre la casa } \\
\dot{¿} ?\end{array}$ & 1 FANEGA DE LINAZA & & $\begin{array}{l}\text { PAGA DE INFURCION } \\
6 \text { HEMINAS DE TRIGO. } \\
\text { HA DE ESTAR EN UN } \\
\text { HEREDERO Y NO } \\
\text { MAS }^{3} \text {. }\end{array}$ \\
\hline $\begin{array}{l}\text { Solar de Ferrando } \\
\text { 1499: HEREDEROS } \\
\text { DEL CLERIGO DE } \\
\text { ARGÜÉBANES, DE } \\
\text { FERRÁN BARÓ Y DE } \\
\text { GUTTIERRE DE } \\
\text { TURIENO } \\
\text { 1515: HEREDEROS } \\
\text { DEL CLERIGO DE } \\
\text { ARGÜÉBANES, }\end{array}$ & $\begin{array}{l}\text { Tienen por préstamos los } \\
\text { dichos tres solares: } \\
1 \text { VIÑA en Mus } \\
6 \text { MIEDROS DE VINO } \\
1 \text { HAZA DE VIÑA en el } \\
\text { Arenal de Mus } \\
1 \text { MIEDRO DE VINO }\end{array}$ & $\begin{array}{l}2 \text { MIEDROS DE VINO } \\
2 \text { MIEDROS DE VINO }\end{array}$ & & $\begin{array}{l}\text { PAGA DE INFURCIÓN } \\
\text { LOS DICHOS SOLARES } \\
\text { Y PRÉSTAMOS } 10 \\
\text { HEMINAS DE TRIGO, } \\
14 \text { AÇUNBRES DE } \\
\text { VINO Y } 3 \text { HUEBRAS } \\
\text { DE BUEYES. ESTA } \\
\text { PUESTO EN TRES } \\
\text { HEREDEROS }\end{array}$ \\
\hline
\end{tabular}

\footnotetext{
${ }^{3}$ APEO 1538: “...HA DE ACUDIR CON LA INFURCIÓN AL SOLAR QUE LLEVA JUAN DE ERMIDA...”
} 


\begin{tabular}{|c|c|c|c|c|}
\hline $\begin{array}{l}\text { ERRÁN VARO Y } \\
\text { UAN DE MARTÍN } \\
\text { 538: LA MUJER DE } \\
\text { UAN DE MARTÍN Y } \\
\text { US HIJOS. }\end{array}$ & $\begin{array}{l}\text { 1 VIÑA en Piedra de Mus } \\
\text { 2-3 CÁNTARAS de VINO } \\
\text { Además de los préstamos que } \\
\text { llevan los herederos del cura } \\
\text { de Argüébanes y sus } \\
\text { consortes (que son la tercera } \\
\text { parte del solar de Ferrando) } \\
\text { llevan las heredades } \\
\text { siguientes: } \\
\text { NO FIGURA } \\
1 \text { VIÑA en Tejalontes } \\
\dot{¿} ? \\
1 \text { VIÑA al Zumacal } \\
\dot{¿} ? \\
1 \text { VIÑA en la Cueva } \\
\dot{¿} ? \\
1 \text { VIÑA en la Pandilla } \\
\dot{¿} ? \\
1 \text { VIÑA en el camino de la } \\
\text { Pandilla } \\
\dot{¿} ? \\
1 \text { VIÑA en el Rejo } \\
\dot{¿} ? \\
1 \text { VIÑA en los Parrales } \\
\dot{\varepsilon} ?\end{array}$ & $\begin{array}{l}\text { HUERTOS Y ERA que } \\
\text { están delante de la casa } \\
\dot{¿} \text { ? } \\
1 \text { TIERRA en Tejalontes } \\
\dot{¿} \text { ? }\end{array}$ & $\begin{array}{l}3 \text { HEMINAS DE LINAZA } \\
1 \text { SESTERO DE TRIGO } \\
1 \text { CÁNTARA DE VINO } \\
1 \text { CÁNTARA DE VINO } \\
\text { NO FIGURA } \\
\text { ¿? } \\
\text { NO FIGURA } \\
2 \text { CÁNTARAS DE VINO }\end{array}$ & $\begin{array}{l}\text { APEO 1538: } \\
\text { INFURCIÓN DEL } \\
\text { SOLAR DE LA } \\
\text { BENITA } 3 \text { HEMINAS Y } \\
1 \text { 1/2 CELEMÍN DE TRIGO, } \\
4 \text { 1⁄2 AZUMBRES DE } \\
\text { VINO, EL TERCIO DE } \\
\text { 1² CELEMÍN Y } 1 \\
\text { HUEBRA DE BUEYES }\end{array}$ \\
\hline
\end{tabular}




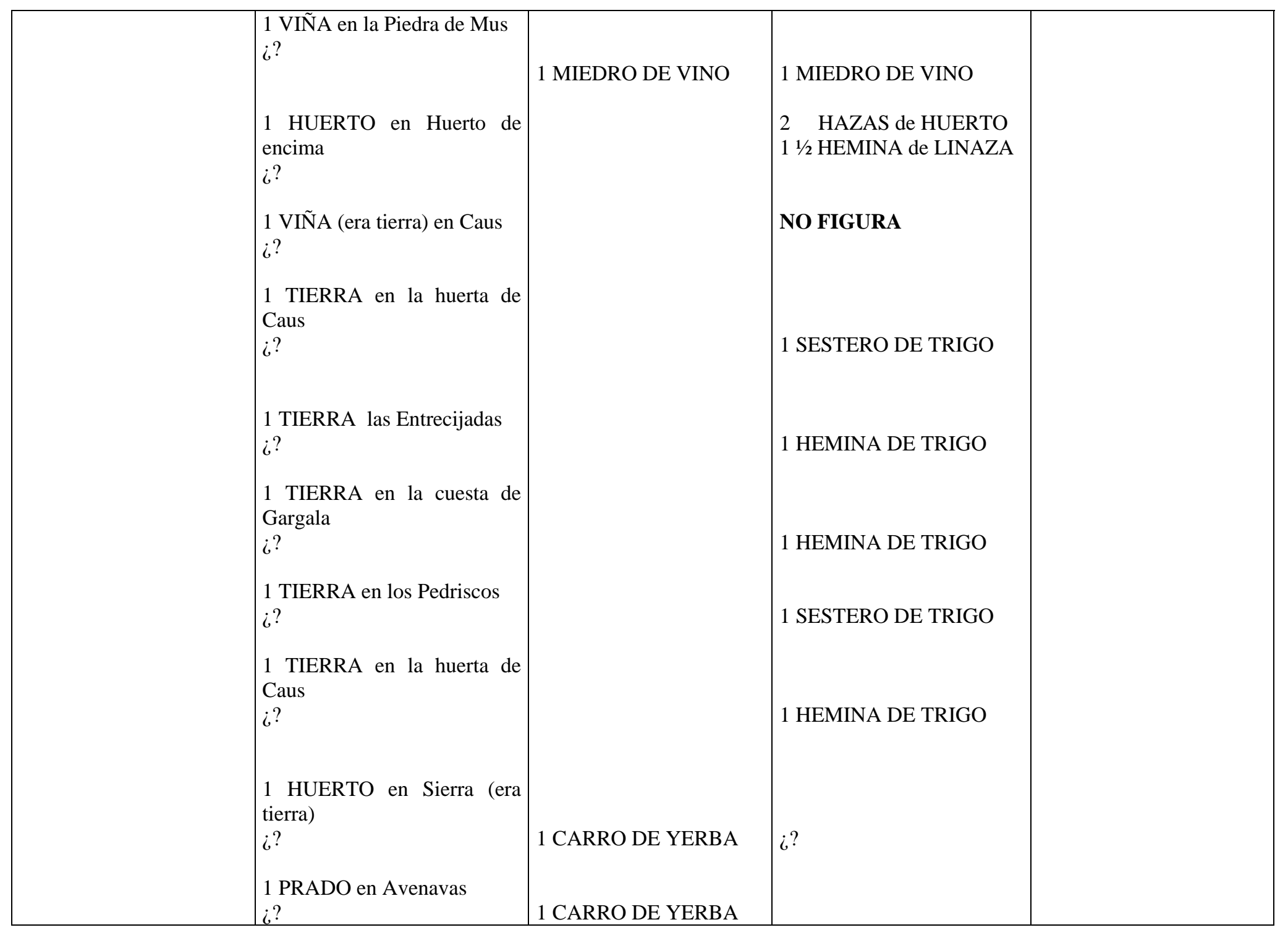




\begin{tabular}{|c|c|c|c|c|}
\hline & 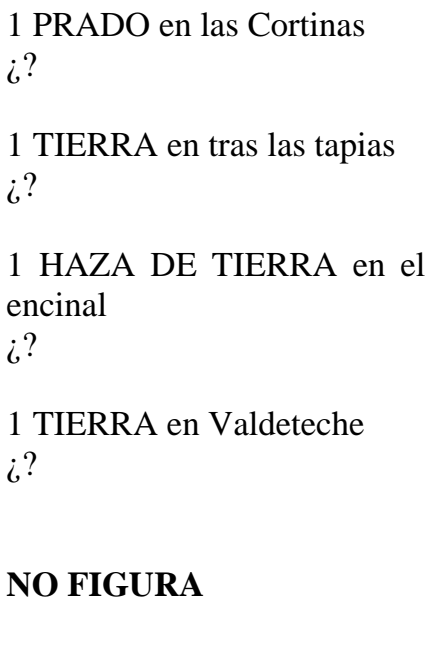 & NO FIGURA & $\begin{array}{l}3 \text { CARGAS DE YERBA } \\
1 \text { HEMINA DE TRIGO } \\
\text { NO FIGURA } \\
\text { TIERRA Y PRADO } \\
1 \text { HEMINA DE TRIGO } \\
2 \text { CARGAS DE YERBA } \\
1 \text { VIÑA a la entrada de Mus } \\
4 \text { CÁNTARAS DE VINO }\end{array}$ & \\
\hline $\begin{array}{l}\text { Solar de Guttierre } \\
\text { 1499: NO FIGURA } \\
\text { 1515: JUAN DE } \\
\text { MARTÍN } \\
\text { 1538: NO FIGURA }\end{array}$ & & $\begin{array}{l}1 \text { VIÑA en Mus en los } \\
\text { Parrales } \\
2 \text { MIEDROS DE VINO } \\
1 \text { VIÑA en Mus } \\
2 \text { CARGAS DE UVAS } \\
1 \text { VIÑA en el Arenal de } \\
\text { Mus } \\
2 \text { CARGAS DE UVAS } \\
1 \text { VIÑA en Mus } \\
2 \text { CARGAS DE UVAS }\end{array}$ & & $\begin{array}{l}\text { APEO DE 1515: } \\
\text { PAGA DE INFURCION } \\
1 \text { CUARTO DE TRIGO, } \\
\text { 1/2 CANTARA DE VINO } \\
\text { Y } 1 \text { HUEBRA DE } \\
\text { BUEYES }\end{array}$ \\
\hline $\begin{array}{l}\text { 1499: Solar de Rodrigo de } \\
\text { Floranes } \\
\text { 1538: Solar de Alonso de }\end{array}$ & $\begin{array}{l}1 \text { VIÑA sobre el molino de } \\
\text { Santo Toribio } \\
2 \text { MIEDROS DE VINO }\end{array}$ & & & $\begin{array}{l}\text { PAGA DE INFURCION } \\
2 \text { FANEGAS Y } 1\end{array}$ \\
\hline
\end{tabular}




\begin{tabular}{|c|c|c|c|c|}
\hline $\begin{array}{l}\text { Llano y Gonzalo de } \\
\text { Floranes. Se dice el solar } \\
\text { de Rodrigo Floranes } \\
\text { 1499: RODRIGO DE } \\
\text { FLORANES } \\
\text { 1515: HEREDEROS DE } \\
\text { RODRIGO DE } \\
\text { FLORANES } \\
\text { 1538: TERESA, mujer de } \\
\text { ALONSO DE LLANO. }\end{array}$ & 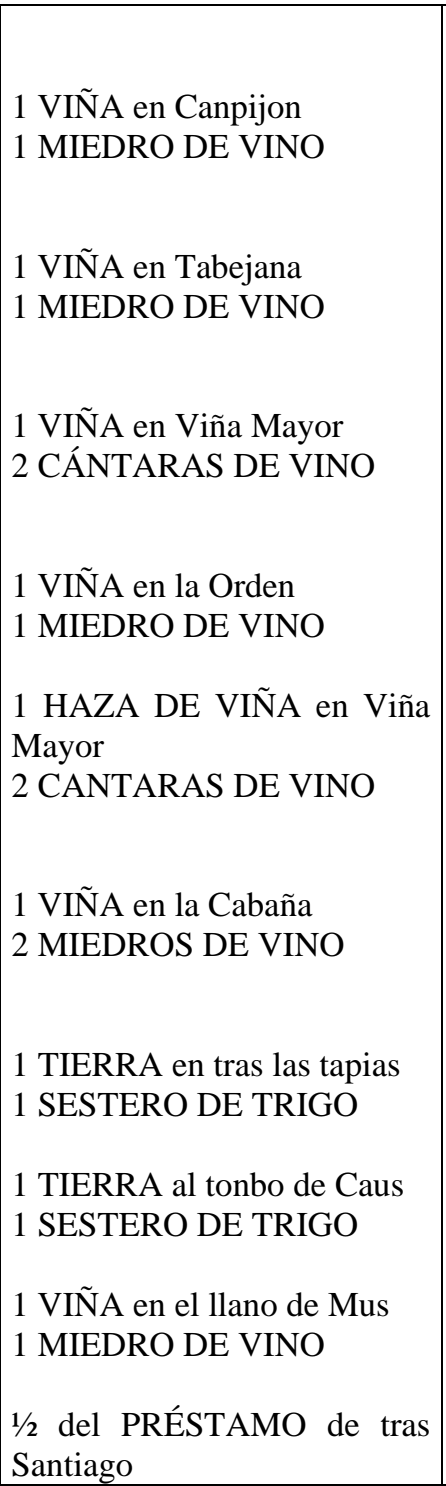 & $\begin{array}{l}\text { 3-4 CÁNTARAS DE } \\
\text { VINO }\end{array}$ & $\begin{array}{l}1 \text { MIEDRO DE VINO } \\
\text { NO FIGURA } \\
1 \text { TIERRA en La Cabaña } \\
1 \text { CUARTO DE PAN } \\
\text { NO FIGURA }\end{array}$ & $\begin{array}{l}\text { SESTERO DE TRIGO, } 3 \\
\text { CÁNTARAS DE VINO } \\
\text { Y } 1 \text { HUEBRA DE } \\
\text { BUEYES }\end{array}$ \\
\hline
\end{tabular}




\begin{tabular}{|c|c|c|c|c|}
\hline & $\begin{array}{l}1 \text { 1⁄2 FANEGA DE TRIGO } \\
1 \text { PRADO en [Honcalvilla] } \\
2 \text { CARROS DE YERBA }\end{array}$ & $\begin{array}{l}1 \text { FANEGA DE TRIGO } \\
\text { 1CARRO DE YERBA los } \\
1 / 2 \text { años }\end{array}$ & & \\
\hline $\begin{array}{l}\text { Solar de Juan de Flores } \\
\text { 1499 y 1515: JUAN DE } \\
\text { FLORES } \\
\text { 1538: NO FIGURA }\end{array}$ & & & & $\begin{array}{l}\text { PAGA DE INFURCIÓN } \\
\text { LOS MEDIOS AÑOS } 3 \\
\text { PANES Y UNA } \\
\text { GALLINA }\end{array}$ \\
\hline $\begin{array}{l}\text { Solar de la Aldega } \\
\text { 1499 y 1515: ALONSO } \\
\text { GÓMEZ DE BARÓ } \\
\text { 1538: PEDRO } \\
\text { GONZÁLEZ DE } \\
\text { TURIENO }\end{array}$ & $\begin{array}{l}1 \text { HUERTO junto a la casa } \\
1 \text { CUARTO DE LINAZA } \\
1 \text { VIÑA al ponton de } \\
\text { Rexondo } \\
2 \text { MIEDROS DE VINO } \\
1 \text { TIERRA Y PRADO a la } \\
\text { fuente del Dobro } \\
1 \text { SESTERO DE TRIGO } \\
2 \text { CARGAS DE YERBA } \\
1 \text { TIERRA en Overan } \\
1 \text { CUARTO DE TRIGO } \\
1 \text { TIERRA sobre Arcillero } \\
1 \text { HEMINA DE TRIGO }\end{array}$ & $\begin{array}{l}1 \text { MIEDRO DE VINO } \\
1 \text { SESTERO DE TRIGO } \\
4 \text { CARGAS DE YERBA }\end{array}$ & $\begin{array}{l}2 \text { MIEDROS DE VINO } \\
\text { NO FIGURA }\end{array}$ & $\begin{array}{l}\text { PAGA DE INFURCION } \\
10 \text { HEMINAS DE } \\
\text { TRIGO, } 14 \text { AZUMBRES } \\
\text { DE VINO Y } 3 \\
\text { HUEBRAS DE BUEYES }\end{array}$ \\
\hline $\begin{array}{l}\text { Solar de Aldonza } \\
\text { 1499: GONZALO DE } \\
\text { FLORANES y OTROS } \\
\text { 1515: JUAN DE BARÓ } \\
\text { DE TURIENO }\end{array}$ & $\begin{array}{l}1 \text { VIÑA en Olinas } \\
2 \text { MIEDROS DE VINO } \\
1 \text { VIÑA en Olinas } \\
3 \text { MIEDROS DE VINO }\end{array}$ & 2 MIEDROS DE VINO & & $\begin{array}{l}\text { PAGA DE INFURCION } \\
6 \text { HEMINAS Y } 1 \\
\text { CELEMÍN DE TRIGO, } 9 \\
\text { AZUMBRES Y } 1 / 2 \text { Y } 1 \\
\text { CUARTILLO DE VINO, }\end{array}$ \\
\hline
\end{tabular}




\begin{tabular}{|c|c|c|c|c|}
\hline $\begin{array}{l}\text { APEO 1538: JUAN DE } \\
\text { BARÓ }\end{array}$ & $\begin{array}{l}1 \text { VIÑA en Tabejana } \\
4 \text { - } 5 \text { CÁNTARAS DE VINO } \\
1 \text { VIÑA en Olinas } \\
4 \text { CÁNTARAS DE VINO } \\
\text { NO FIGURA }\end{array}$ & $\begin{array}{l}1 \text { MIEDRO DE VINO } \\
\text { siendo bien plantada } \\
\text { NO FIGURA } \\
\text { NO FIGURA }\end{array}$ & $\begin{array}{l}4 \text { - } 5 \text { CÁNTARAS DE } \\
\text { VINO } \\
\text { NO FIGURA } \\
1 \text { VIÑA en Tabejana } \\
2 \text { CÁNTARAS DE VINO }\end{array}$ & $\begin{array}{l}2 \text { HUEBRAS DE } \\
\text { BUEYES } \\
\text { APEO 1538: } 9 \text { azumbres } \\
\text { y } 1 \text { cuartillo de vino. }\end{array}$ \\
\hline $\begin{array}{l}\text { 1499 y 1515: El solar de } \\
\text { Juan de Varo } \\
\text { 1538: Solar de Inés } \\
\text { González } \\
\text { 1499: JUAN DE BARÓ } \\
\text { 1515: Hijos de } \\
\text { FERRANDO LÓPEZ DE } \\
\text { POTES y la mujer de } \\
\text { DIEGO DE LINARES } \\
\text { 1538: INÉS GONZÁLEZ }\end{array}$ & $\begin{array}{l}1 \text { HAZA HUERTO en los } \\
\text { Gallaranes. } \\
1 \text { HEMINA DE LINAZA } \\
1 \text { VIÑA en Tabejana, que es } \\
\text { en Llan de Viñas } \\
4 \text { MIEDROS DE VINO } \\
1 \text { VIÑA en Llandeçilla } \\
2 \text { MIEDROS DE VINO } \\
1 \text { VIÑA sobre Lodias } \\
2 \text { MIEDROS DE VINO }\end{array}$ & & 8 MIEDROS DE VINO & $\begin{array}{l}\text { PAGA DE INFURCION } \\
1 \text { FANEGA DE TRIGO, } \\
2 \text { CÁNTARAS y 1/2 DE } \\
\text { VINO Y } 1 \text { GALLINA, la } \\
\text { cual dicha gallina pagaba } \\
\text { el solar antes de que se } \\
\text { hiciese el troque }\end{array}$ \\
\hline $\begin{array}{l}\text { 1499: Solar de Alonso de } \\
\text { la Fuente } \\
1515 \text { y 1538: Solar de }\end{array}$ & $\begin{array}{l}1 \text { VIÑA en Olinas } \\
2 \text { MIEDROS DE VINO }\end{array}$ & & & \\
\hline
\end{tabular}

\footnotetext{
${ }^{4}$ APEO DE 1515: Diose en enmienda por esta tierra una viña en Mus que hay 2 MIEDROS DE VINO.
} 


\begin{tabular}{|c|c|c|c|c|}
\hline \begin{tabular}{|l|} 
Juan de la Fuente \\
1499: ALONSO DE LA \\
FUENTE \\
1515 y 1538: JUAN DE \\
LA FUENTE
\end{tabular} & $\begin{array}{l}2 \text { HAZAS DE VIÑA en San } \\
\text { Lázaro } \\
3 \text { MIEDROS DE VINO } \\
1 \text { TIERRA en [Otero] }{ }^{4} \\
1 \text { CUARTO DE TRIGO } \\
\\
1 \text { TIERRA en Alzapierna } \\
1 \text { HEMINA DE TRIGO } \\
1 \text { VIÑA en Tabejana } \\
4 \text { CÁNTARAS DE VINO } \\
1 \text { HUERTO en Otero } \\
1 \text { HEMINA DE LINAZA }\end{array}$ & $\begin{array}{l}1 \text { VIÑA en Alzapierna } \\
\text { (que solía ser tierra) } \\
1 \text { MIEDRO DE VINO }\end{array}$ & $\begin{array}{l}1 \text { VIÑA en Mus } \\
2 \text { MIEDROS DE VINO } \\
4 \text { CÁNTARAS DE VINO } \\
1 \text { VIÑA en Llan de viñas } \\
2 \text { CÁNTARAS DE VINO } \\
\text { NO FIGURA }\end{array}$ & $\begin{array}{l}\text { PAGA DE INFURCION } \\
1 \text { FANEGA DE TRIGO, } \\
1 \text { CÁNTARA Y } 1 / 2 \text { DE } \\
\text { VINO Y } 3 \text { HUEBRAS } \\
\text { DE BUEYES. HA DE } \\
\text { ESTAR EN } \\
\text { HEREDEROS Y NO } \\
\text { MÁS. }\end{array}$ \\
\hline $\begin{array}{l}\text { 1499: Solar de la Fuente } \\
\text { 1515: Solar de Juan } \\
\text { Guttierre de La Fuente } \\
\text { 1538: Solar de Alonso de } \\
\text { la Huente } \\
\text { 1499: JUAN GUTIERRE } \\
\text { DE LA FUENTE } \\
\text { 1515: PEDRO DE LA } \\
\text { FUENTE } \\
\text { 1538: JUAN DE LA } \\
\text { FUENTE Y PEDRO DE } \\
\text { VILLANUEVA }\end{array}$ & $\begin{array}{l}1 \text { HUERTO junto al solar } \\
1 \text { SESTERO DE LINAZA } \\
1 \text { TIERRA en la llama de } \\
\text { SIerra } \\
1 \text { FANEGA DE TRIGO }\end{array}$ & $\begin{array}{l}3 \text { HEMINAS de LINAZA } \\
1 \text { TIERRA en Trascaldillo } \\
3 \text { CUARTOS DE PAN }\end{array}$ & $\begin{array}{l}1 \text { TIERRA y PRADO en la } \\
\text { llama de la Sierra } \\
3 \text { HEMINAS DE TRIGO y } \\
1 \text { CARRO DE YERBA }\end{array}$ & $\begin{array}{l}\text { PAGA DE INFURCION } \\
5 \text { HEMINAS DE TRIGO, } \\
14 \text { AZUMBRES DE } \\
\text { VINO Y } 3 \text { HUEBRAS } \\
\text { DE BUEYES }\end{array}$ \\
\hline
\end{tabular}




\begin{tabular}{|c|c|c|c|}
\hline $\begin{array}{l}\text { 1499: NO FIGURA } \\
1515 \text { y 1538: Solares de } \\
\text { Orejon }\end{array}$ & & $\begin{array}{l}\text { Todas las heredades y } \\
\text { solares que llevaba Orejon } \\
\text { en término re de } \\
\text { Santybannes las cuales } \\
\text { llevan ahora muchas } \\
\text { personas por el señor } \\
\text { duque que las entro. }\end{array}$ & $\begin{array}{l}\text { PAGABA OREJON DE } \\
\text { INFURCION } 1 \\
\text { CARNERO VIVO, } 1 \\
\text { CUARTO DE TRIGO, } 1 \\
\text { CANTARA DE VINO Y } \\
1 \text { PAR DE CAPONES } \\
\text { SIN DESCUENTO } \\
\text { ALGUNO. } \\
\text { PAGA EL SEÑOR } \\
\text { DUQUE AL PRESENTE } \\
\text { 150 MRS. Y } 200 \text { MRS. } \\
\text { DE LIMOSNA }\end{array}$ \\
\hline $\begin{array}{l}\text { 1515: Solar de [Toribio] } \\
\text { de Pedro Otero } \\
\text { 1499: PEDRO DE } \\
\text { OTERO } \\
\text { 1515: TORIBIO DE } \\
\text { PEDRO OTERO } \\
\text { 1538: NO FIGURA }\end{array}$ & $\begin{array}{l}1 \text { HUERTO } \\
1 / 2 \text { HEMINA DE LINAZA }\end{array}$ & & $\begin{array}{l}\text { PAGA DE INFURCIÓN } \\
1 \text { HEMINA DE TRIGO }\end{array}$ \\
\hline $\begin{array}{l}\text { 1499: Solar de García } \\
\text { Ponce } \\
\text { 1499: GARCÍA PONCE } \\
\text { 1515 y 1538: NO } \\
\text { FIGURA }\end{array}$ & $\begin{array}{l}1 \text { VIÑA en Olinas } \\
1 \text { MIEDRO DE VINO } \\
1 \text { VIÑA en Viña Mayor } \\
1 \text { MIEDRO DE VINO }\end{array}$ & & $\begin{array}{l}\text { 1499: PAGA DE } \\
\text { INFURCIÓN } 5 \\
\text { HEMINAS DE TRIGO, } 7 \\
\text { AZUMBRES DE VINO y } \\
1 \text { 1⁄2 HUEBRAS DE } \\
\text { BUEYES }\end{array}$ \\
\hline 1499: Solar de Gonzalo & 1 TIERRA al Palomar & & \\
\hline
\end{tabular}




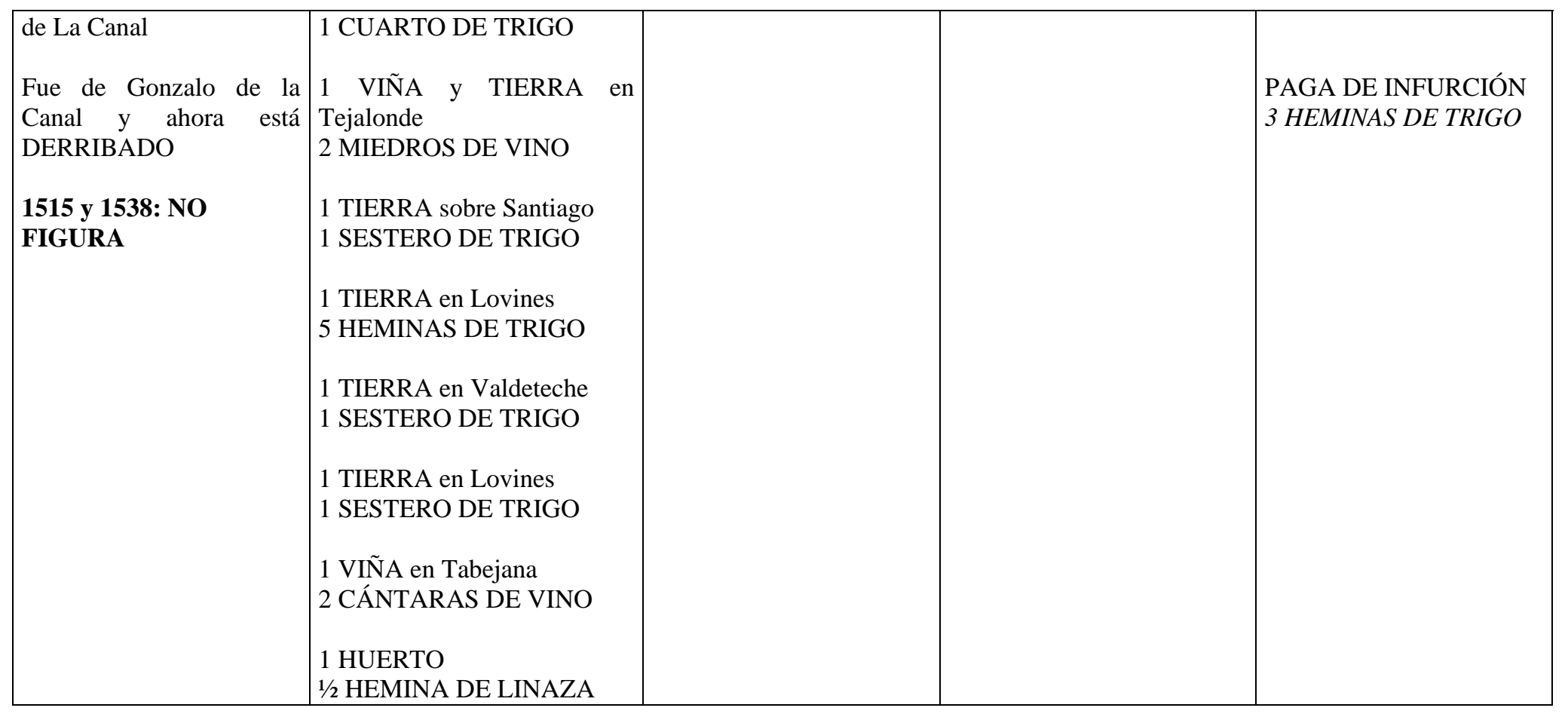

\section{SOLARES EN FLORANES}

\begin{tabular}{|l|l|l|l|}
\hline \multicolumn{1}{|c|}{ TENENTE } & \multicolumn{1}{|c|}{1499} & 1515 & 1538 \\
\hline $\begin{array}{l}\text { FLORANES. Solar del } \\
\text { Troche }\end{array}$ & $\begin{array}{l}\text { 1 VIÑA en el llano de Mus } \\
\text { 6 MIEDROS DE VINO }\end{array}$ & 7-8 MIEDROS DE VINO & RENTA \\
$\begin{array}{l}\text { 1499: DIEGO } \\
\text { FERNÁNDEZ DE }\end{array}$ & 1 VIÑA en Olinas & PAGA DE INFURCION \\
14 HEMINAS DE \\
TRIGO, 28 AZUMBRES
\end{tabular}




\begin{tabular}{|c|c|c|c|c|}
\hline $\begin{array}{l}\text { FLORANES } \\
\text { 1515: Hijos de DIEGO } \\
\text { FERNÁNDEZ DE } \\
\text { FLORANES } \\
\text { 1538: JUAN DÍAZ DE } \\
\text { FLORANES y sus } \\
\text { CONSORTES. }\end{array}$ & $\begin{array}{l}12 \text { CÁNTARAS DE VINO } \\
1 \text { 14 TIERRA de tras Santiago } \\
5 \text { HEMINAS DE TRIGO } \\
1 \text { SOLAR en Mieses que está } \\
\text { hecho casar } \\
1 \text { PEDAZO del Campo de } \\
\text { Armental con sus árboles } \\
1 \text { VIÑA en Valladar } \\
3 \text { MIEDROS DE VINO } \\
1 \text { TIERRA en Mieses } \\
5 \text { HEMINAS DE TRIGO }\end{array}$ & $\begin{array}{l}2 \text { MIEDROS DE VINO } \\
1 \text { CUARTO DE TRIGO } \\
5 \text { MIEDROS DE VINO } \\
6 \text { HEMINAS DE TRIGO }\end{array}$ & $\begin{array}{l}10 \text { CÁNTARAS DE VINO } \\
5 \text { HEMINAS DE TRIGO } \\
3 \text { MIEDROS DE VINO } \\
1 \text { VIÑA en Mieses } \\
4 \text { MIEDROS DE VINO }\end{array}$ & $\begin{array}{l}\text { DE VINO, } 3 \text { HUEBRAS } \\
\text { DE BUEYES Y } 1 \\
\text { GALLINA. } \\
\text { HA DE QUEDAR EN } \\
\text { TRES HEREDEROS Y } \\
\text { NO MAS. }\end{array}$ \\
\hline $\begin{array}{l}\text { FLORANES Solar de } \\
\text { María González } \\
\text { 1499 y 1515: } \\
\text { FERRANDO VARO } \\
\text { 1538: JUAN DE } \\
\text { RODRÍGUEZ, NIETO } \\
\text { DE FERNANDO DE } \\
\text { BARÓ }\end{array}$ & $\begin{array}{l}1 \text { VIÑA en el Troche } \\
3 \text { MIEDROS DE VINO } \\
1 \text { VIÑA en Mus } \\
10 \text { CÁNTARAS DE VINO } \\
1 \text { haza de VIÑA en Mus } \\
1 \text { MIEDRO DE VINO } \\
\text { NO FIGURA }\end{array}$ & $\begin{array}{l}2 \text { MIEDROS DE VINO } \\
1 \text { HUERTO sobre la torre } \\
\text { de Floranes } \\
¿ \text { ? }\end{array}$ & & $\begin{array}{l}\text { PAGA } 1 \text { FANEGA DE } \\
\text { TRIGO, } 1 / 2 \text { CANTARA } \\
\text { DE VINO Y } 1 \text { HUEBRA } \\
\text { DE BUEYES }\end{array}$ \\
\hline FLORANES & & & & \\
\hline
\end{tabular}

${ }^{5}$ HA DE QUEDAR EN 1 HEREDERO Y NO MAS. HASTA POR TRES VIDAS

APEO 1538: Agrega: "en esta manera del solar de Mari Gonçalez 1 quarto de trigo" 


\begin{tabular}{|c|c|c|c|c|}
\hline $\begin{array}{l}\text { (1) 1499: Solar de } \\
\text { Gonzalo de Floranes } \\
\text { 1538: Solar de Rodrigo y } \\
\text { Gonçalo de Floranes } \\
\text { 1499 Y 1515: GONZALO } \\
\text { DE FLORANES } \\
\text { 1538: RODRIGO DE } \\
\text { FLORANES y } \\
\text { GONZALO DE } \\
\text { FLORANES } \\
\text { (2) 1499: Solar de Juan de } \\
\text { Treceño. } \\
\text { 1515 Y 1538: Solar de } \\
\text { García Treceño. } \\
\text { 1499: JUAN DE } \\
\text { TRECEÑO } \\
\text { 1515: HIJOS DE JUAN } \\
\text { DE TRECEÑO } \\
\text { 1538: HIJOS DE } \\
\text { GARCÍA DE TRECEÑO }\end{array}$ & $\begin{array}{l}1 \text { VIÑA en Mus } \\
3 \text { a } 4 \text { MIEDROS DE VINO } \\
1 \text { VIÑA en Olinas } \\
5 \text { MIEDROS DE VINO } \\
1 \text { VIÑA en la Cueva } \\
2 \text { MIEDROS DE VINO } \\
1 \text { VIÑA en Campejón, en el } \\
\text { cuerano de Tabejana } \\
3 \text { MIEDROS DE VINO } \\
1 \text { VIÑA en Olinas } \\
2 \text { MIEDROS DE VINO } \\
1 \text { VIÑA en Caus } \\
3 \text { MIEDROS DE VINO } \\
1 \text { VIÑA Trascasa en el } \\
\text { Troche } \\
1 \text { MIEDRO DE VINO } \\
1 \text { VIÑA en el Troche } \\
4-5 \text { CÁNTARAS DE VINO }\end{array}$ & $\begin{array}{l}1 \text { VIÑA y TIERRA en } \\
\text { Caus } \\
4 \text { MIEDROS DE VINO, } \\
\text { estando toda plantada }\end{array}$ & $\begin{array}{l}1 \text { TIERRA en Caus } \\
1 \text { FANEGA DE TRIGO } \\
\text { NO FIGURA } \\
\text { NO FIGURA }\end{array}$ & $\begin{array}{l}\text { PAGAN } 2 \text { FANEGAS DE } \\
\text { TRIGO Y } 1 \text { CANTARA } \\
\text { DE VINO, más } 1 \\
\text { SESTERO DE TRIGO } \\
\text { por la viña de la Cueva } \\
\text { que era del solar de } \\
\text { María Gonzalez. } \\
\text { "Asi es la infurcion que } \\
\text { han de pagar los } \\
\text { susodichos y sus } \\
\text { CONSORTES" }\end{array}$ \\
\hline
\end{tabular}




\section{SOLARES EN OTERO}

\begin{tabular}{|c|c|c|c|c|}
\hline LUGAR & 1499 & 1515 & 1538 & RENTA \\
\hline $\begin{array}{l}\text { 1499: Solar de Diego } \\
\text { Martínez } \\
\text { 1499 y 1515: DIEGO } \\
\text { MARTÍNEZ DE BARÓ } \\
\text { 1538: ALONSO y } \\
\text { DIEGO, hijos de } \\
\text { (ilegible) DE BARÓ }\end{array}$ & $\begin{array}{l}1 \text { VIÑA en la Orden } \\
4 \text { MIEDROS DE VINO } \\
1 \text { HAZA DE PRADO en } \\
\text { Barriberó } \\
1 \text { CARRO DE YERBA } \\
1 \text { VIÑA en la Molina } \\
2 \text { CÁNTARAS DE VINO } \\
1 \text { VIÑA en Llan de Viñas } \\
1 \text { CÁNTARA DE VINO } \\
1 \text { HAZA DE VIÑA en } \\
\text { Camares } \\
1 \text { CÁNTARA DE VINO } \\
1 \text { HUERTO en Otero } \\
1 \text { HEMINA DE LINAZA } \\
1 \text { HAZA DE VIÑA en Viña } \\
\text { Mayor } \\
2 \text { CANTARAS DE VINO } \\
1 \text { HUERTO en Otero } \\
1 \text { HEMINA DE LINAZA }\end{array}$ & $\begin{array}{l}5 \text { MIEDROS DE VINO } \\
2 \text { CARROS DE YERBA } \\
3 \text { CÁNTARAS DE VINO } \\
2 \text { CÁNTARAS DE VINO } \\
2 \text { CÁNTARAS DE VINO } \\
1 \text { SESTERO DE LINAZA }\end{array}$ & 2 CÁNTARAS DE VINO & $\begin{array}{l}\text { PAGA DE INFURCIÓN } \\
3 \text { HEMINAS DE TRIGO } \\
\text { Y 1/2 CÁNTARA DE } \\
\text { VINO }\end{array}$ \\
\hline $\begin{array}{l}\text { Solar de Pedro de Otero } \\
\text { 1499: PEDRO DE } \\
\text { OTERO }\end{array}$ & $\begin{array}{l}1 \text { VIÑA en la Cabaña } \\
2 \text { MIEDROS DE VINO }\end{array}$ & 4 MIEDROS DE VINO & & \\
\hline
\end{tabular}




\begin{tabular}{|c|c|c|c|c|}
\hline $\begin{array}{l}\text { 1515: TORIBIO DE } \\
\text { PEDRO DE OTERO y } \\
\text { sus consortes. } \\
\text { 1538: TERESA DE } \\
\text { OTERO }\end{array}$ & $\begin{array}{l}1 \text { PRADO en las Cortinas de } \\
\text { Argüébanes } \\
2 \text { CARGAS DE YERBA } \\
1 \text { VIÑA en Camares } \\
4 \text { CÁNTARAS DE VINO } \\
1 \text { PANEDO en Valcalvo } \\
\text { ¿? } \\
1 \text { VIÑA en Viña Mayor } \\
1 \text { CÁNTARA DE VINO } \\
1 \text { HAZA DE VIÑA en la } \\
\text { cuesta de Tabejana } \\
1 \text { CÁNTARA DE VINO }\end{array}$ & $\begin{array}{l}3 \text { CARGAS DE YERBA } \\
1 \text { MIEDRO DE VINO } \\
1 \text { VIÑA en Valcalvo } \\
2 \text { CARGAS DE UVAS } \\
3 \text { CÁNTARAS DE VINO }\end{array}$ & $\begin{array}{l}2 \text { CARGAS DE YERBA } \\
1 \text { PANEDO en Valcavo } \\
\dot{¿} ?\end{array}$ & $\begin{array}{l}\text { PAGA DE INFURCIÓN } \\
10 \text { HEMINAS DE } \\
\text { TRIGO Y } 10 \\
\text { AZUMBRES DE VINO. } \\
\text { HA DE QUEDAR EN } \\
\text { DOS HEREDEROS Y } \\
\text { NO MAS. }\end{array}$ \\
\hline $\begin{array}{l}\text { 1499: JUAN LÓPEZ DE } \\
\text { ARMAÑO } \\
1515 \text { Y 1538: JUAN DE } \\
\text { BARÓ }\end{array}$ & $\begin{array}{l}1 \text { VIÑA y PRADO en } \\
\text { Hondón del Valle } \\
1 \text { 1/2 CARRO DE YERBA } \\
\text { 5-6-MIEDROS DE VINO } \\
1 \text { TIERRA en Arçenilla } \\
1 \text { FANEGA DE TRIGO }\end{array}$ & $\begin{array}{l}1 \text { VIÑA y HUERTO en } \\
\text { Hondón del Valle } \\
3 \quad \text { CUARTOS DE } \\
\text { LINAZA } 10 \text { MIEDROS } \\
\text { DE VINO }\end{array}$ & $\begin{array}{l}1 \text { VIÑA y TIERRA en } \\
\text { Otero } \\
6 \text { HEMINAS DE TRIGO } \\
4 \text { MIEDROS DE VINO }\end{array}$ & $\begin{array}{l}\text { PAGA DE INFURCION } \\
2 \text { FANEGAS DE TRIGO }\end{array}$ \\
\hline $\begin{array}{l}\text { OTERO. Solar del cura. } \\
\text { 1499: HEREDEROS DE } \\
\text { JUAN DE GARCÍA, cura } \\
\text { de Potes, que son: JUAN } \\
\text { DE ARMAÑO, } \\
\text { GONZALO GÓMEZ y } \\
\text { ALONSO GÓMEZ DE } \\
\text { BARÓ }\end{array}$ & $\begin{array}{l}1 \text { VIÑA en Llan de Viñas } \\
3 \text { MIEDROS DE VINO } \\
1 \text { VIÑA en la Molina } \\
8 \text { CÁNTARAS DE VINO } \\
1 \text { HAZA DE VIÑA en la }\end{array}$ & $\begin{array}{l}4 \text { MIEDROS DE VINO } \\
2 \text { MIEDROS DE VINO }\end{array}$ & & $\begin{array}{l}\text { PAGA DE INFURCION } \\
10 \text { HEMINAS DE } \\
\text { TRIGO Y } 14 \\
\text { AZUMBRES DE VINO }\end{array}$ \\
\hline
\end{tabular}




\begin{tabular}{|c|c|c|c|c|}
\hline $\begin{array}{l}\text { 1515: GARCÍA GÓMEZ, } \\
\text { MARTÍN BORES, } \\
\text { GARCÍA BORES Y } \\
\text { TORIBIO DE BARÓ } \\
\text { (herederos de Juan } \\
\text { García, cura de Potes) } \\
\text { 1538: BARTOLOMÉ DE } \\
\text { OTERO y GARCÍA DE } \\
\text { BORES }\end{array}$ & \begin{tabular}{|l|} 
Molina \\
1 MIEDRO DE VINO \\
1 VIÑA en la Molina \\
10 CÁNTARAS DE VINO \\
1 HAZA DE VIÑA en \\
Llandecilla \\
4 CÁNTARAS DE VINO \\
1 VIÑA en Viña Mayor \\
2 CÁNTARAS DE VINO \\
1 VIÑA en Viña Mayor \\
2 CÁNTARAS DE VINO
\end{tabular} & $\begin{array}{l}8 \text { CÁNTARAS DE VINO } \\
1 \text { MIEDRO DE VINO } \\
1 \text { MIEDRO DE VINO }\end{array}$ & $\begin{array}{l}2 \text { CÁNTARAS DE VINO } \\
\text { NO FIGURA }\end{array}$ & \\
\hline $\begin{array}{l}\text { Solar de Mesayna } \\
\text { 1499: GARCÍA DE } \\
\text { OTERO, GONZALO DE } \\
\text { BARÓ, merino, y } \\
\text { OTROS. } \\
\text { 1515: ALONSO, hijo de } \\
\text { FERNANDO DE BARÓ } \\
\text { y ELVIRA, mujer de } \\
\text { GARCÍA GONZÁLEZ } \\
\text { DE BULNES. } \\
\text { 1538: JUAN DEL } \\
\text { MERINO y los herederos } \\
\text { de FERNANDO DE } \\
\text { BARÓ }\end{array}$ & $\begin{array}{l}\text { NO FIGURA } \\
1 \text { VIÑA y TIERRA en la } \\
\text { huerta de Mesayna } \\
1 \text { FANEGA DE TRIGO } \\
5 \text { MIEDROS DE VINO } \\
1 \text { PRADO y TIERRA en } \\
\text { Arcinal } \\
\text { ¿? } \\
1 \text { TIERRA en Carrera Nueva } \\
2 \text { FANEGAS DE PAN } \\
1 \text { TIERRA en Barriberó, en } \\
\text { Fuentes } \\
1 \text { FANEGA DE PAN }\end{array}$ & $\begin{array}{l}1 \text { HUERTO en Otero } \\
1 \text { SESTERO DE LINAZA } \\
1 \text { FANEGA DE TRIGO } \\
6-7 \text { MIEDROS DE } \\
\text { VINO } \\
1 \text { 1/2 FANEGA DE PAN } \\
3 \text { CARGAS DE YERBA }\end{array}$ & & $\begin{array}{l}\text { PAGA DE INFURCIÓN } \\
1 \text { FANEGA DE TRIGO } \\
\text { Y } 12 \text { AZUMBRES DE } \\
\text { VINO }\end{array}$ \\
\hline
\end{tabular}




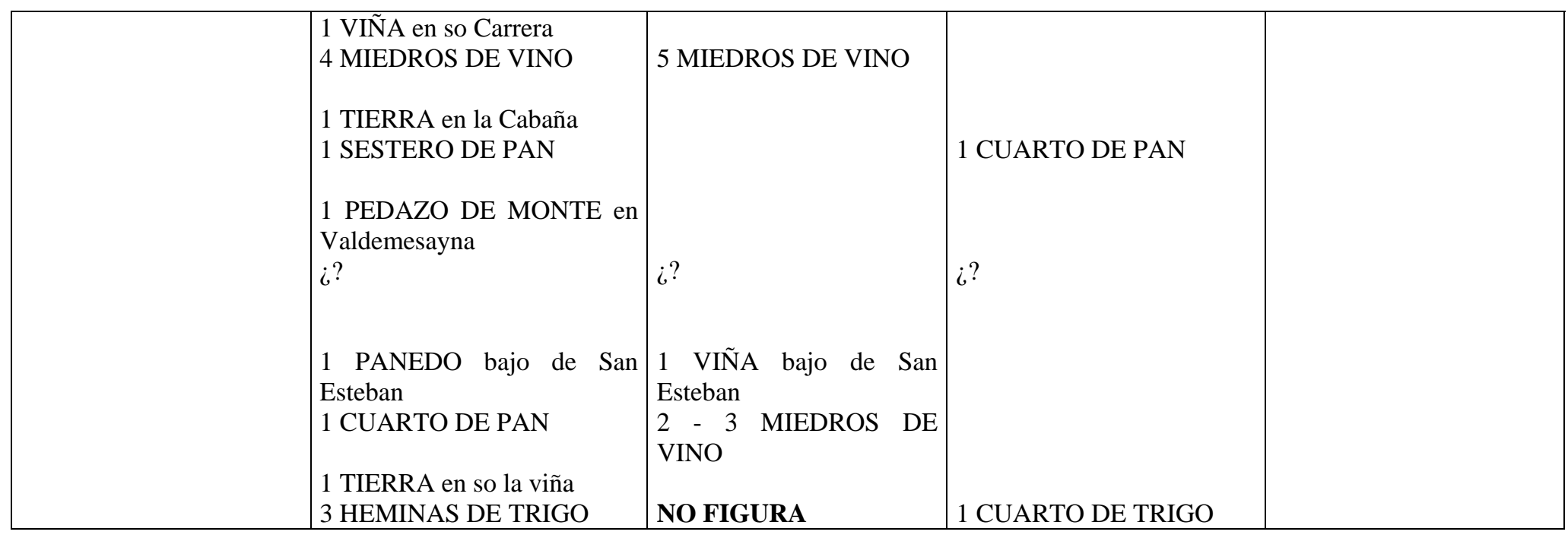

SOLARES EN OJEDO

\begin{tabular}{|c|c|c|c|c|}
\hline TENENTE & 1499 & 1515 & 1538 & RENTA \\
\hline $\begin{array}{l}\text { Solar en Hondón de Villa } \\
\text { La mitad lo lleva JUAN } \\
\text { GRANDE y la otra mitad } \\
\text { HEREDEROS DE } \\
\text { MARIA, mujer que fue de } \\
\text { PEDRO DE MONJE, que } \\
\text { son: TORIBIO, el herrero } \\
\text { de Aliezo, JUAN PEREZ } \\
\text { ROLDAN DE OJEDO y } \\
\text { JUAN MATO DE }\end{array}$ & $\begin{array}{l}1 \text { TIERRA en Ojedo } \\
1 \text { SESTERO DE PAN } \\
1 \text { VIÑA cabo la puente de } \\
\text { Ojedo } \\
1 \text { MIEDRO DE VINO } \\
1 \text { VIÑA en Piedra Aguda }\end{array}$ & $\begin{array}{l}1 \text { TIERRA que liega al } \\
\text { Molino de Juan Mato } \\
1 \text { SESTERO DE PAN }\end{array}$ & 2-3 CARGAS DE UVAS & $\begin{array}{l}\text { PAGA DE INFURCION } \\
6 \text { HEMINAS DE TRIGO } \\
\text { Y CENTENO DE POR } \\
\text { MEDIO Y } 1 \text { CÁNTARA } \\
\text { DE VINO. }\end{array}$ \\
\hline
\end{tabular}




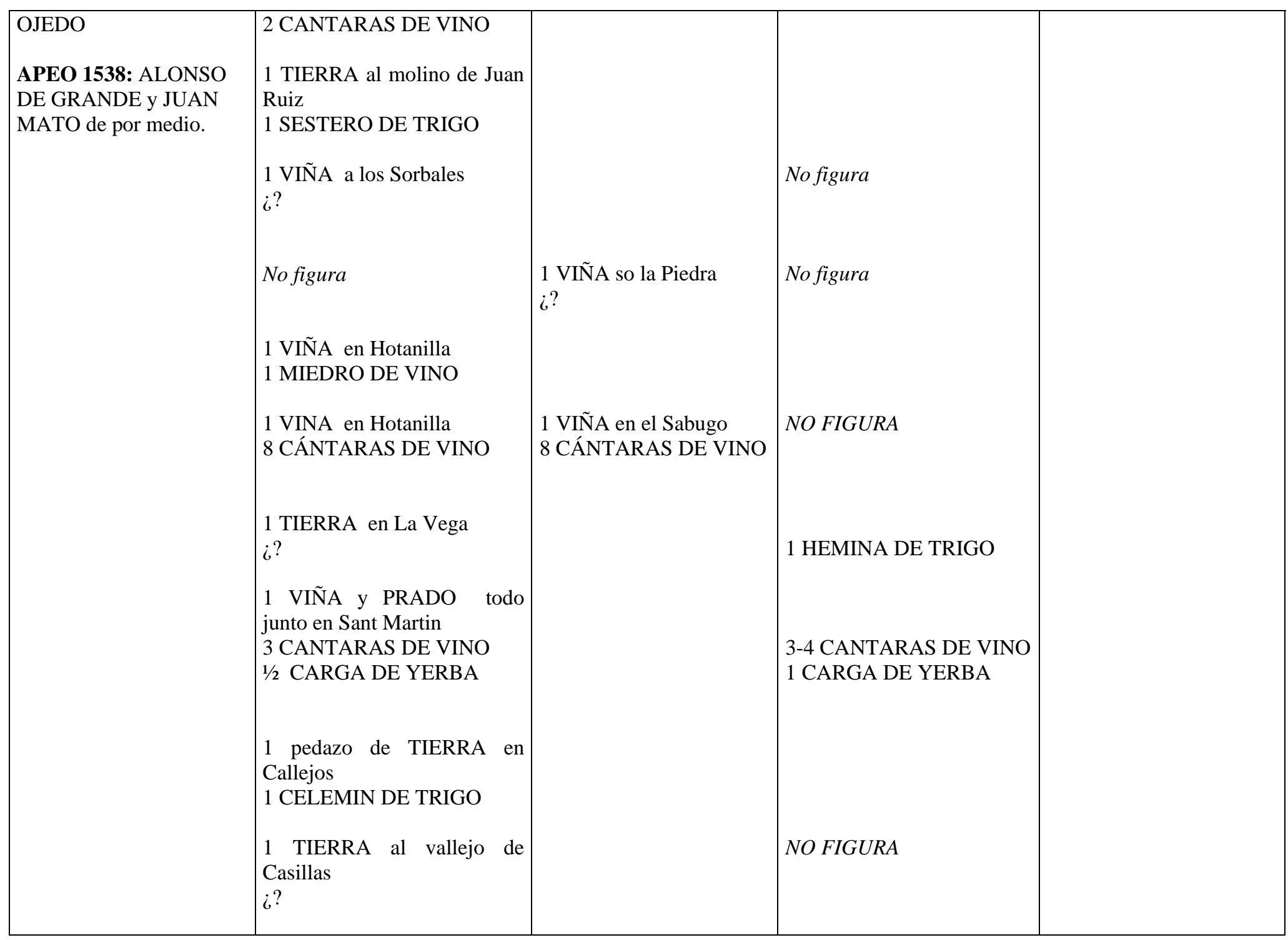




\begin{tabular}{|c|c|c|c|c|}
\hline & $\begin{array}{l}1 \text { HUERTO en Hondón de } \\
\text { Villa de Ojedo } \\
1 \text { CELEMIN TOLEDANO } \\
1 \text { HAZA DE VIÑA en el } \\
\text { Cascajal } \\
2 \text { CANTARAS DE VINO } \\
1 \text { VIÑA en Fontanilla } \\
\text { 3-4 CANTARAS DE VINO }\end{array}$ & & $\begin{array}{l}1 \text { CELEMIN DE TRIGO } \\
\text { NO FIGURA }\end{array}$ & \\
\hline $\begin{array}{l}\text { Solar del Otero } \\
\text { 1/2 DEL SOLAR: } \\
\text { ALONSO DEL OTERO, } \\
\text { FERRANDO DEL } \\
\text { OTERO, JUAN DE } \\
\text { CASTRO, LA MUGER } \\
\text { DE TORIBIO RIOS, } \\
\text { JUAN DE LA CUEVA Y } \\
\text { JUAN FIJO DE JUAN } \\
\text { ROYS } \\
\text { LA OTRA } 1 / 2 \text { : JUAN } \\
\text { GRANDE, JUAN DE } \\
\text { COLLO, TORIBIO DEL } \\
\text { CURERO, JUANA } \\
\text { MUGER QUE FUE DE } \\
\text { ALONSO CASTRO, } \\
\text { JUAN DE LAS CUEVAS } \\
\text { Y FIJOS DE JUAN DE } \\
\text { LA POSADA DE } \\
\text { MIESES }\end{array}$ & $\begin{array}{l}1 \text { TIERRA sobre el molino. } \\
\dot{¿} \text { ? } \\
1 \text { PRADO junto con el dicho } \\
\text { solar } \\
3-4 \text { CARGAS DE YERBA } \\
1 \text { VIÑA Y PRADO en la } \\
\text { Hortalla } \\
6 \text { MIEDROS DE VINO } \\
1 \text { CARGA DE YERBA } \\
\\
\\
1 \text { VIÑA en la [Hortalla] } \\
\text { 5-6 MIEDROS DE VINO }\end{array}$ & $\begin{array}{l}1 \text { VIÑA en la Hortalla } \\
6 \text { MIEDROS DE VINO }\end{array}$ & $\begin{array}{l}6 \text { HEMINAS DE PAN } \\
1 \text { VIÑA en San Martín } \\
6 \text {-7 MIEDROS DE VINO } \\
1 \text { VIÑA en la Fontanilla } \\
6 \text { MIEDROS DE VINO }\end{array}$ & $\begin{array}{l}\text { PAGA EL DICHO } \\
\text { SOLAR Y PRESTAMOS } \\
6 \text { HEMINAS DE PAN } \\
\text { LA MITAD TRIGO Y } \\
\text { LA MITAD CENTENO } \\
\text { Y } 1 \text { CÁNTARA DE } \\
\text { VINO. } \\
\text { FALLASE AL } \\
\text { PRESENTE SEIS } \\
\text { POBLADORES EN EL } \\
\text { DICHO SOLAR. } \\
\text { APEO 1538: PAGA } 3\end{array}$ \\
\hline
\end{tabular}




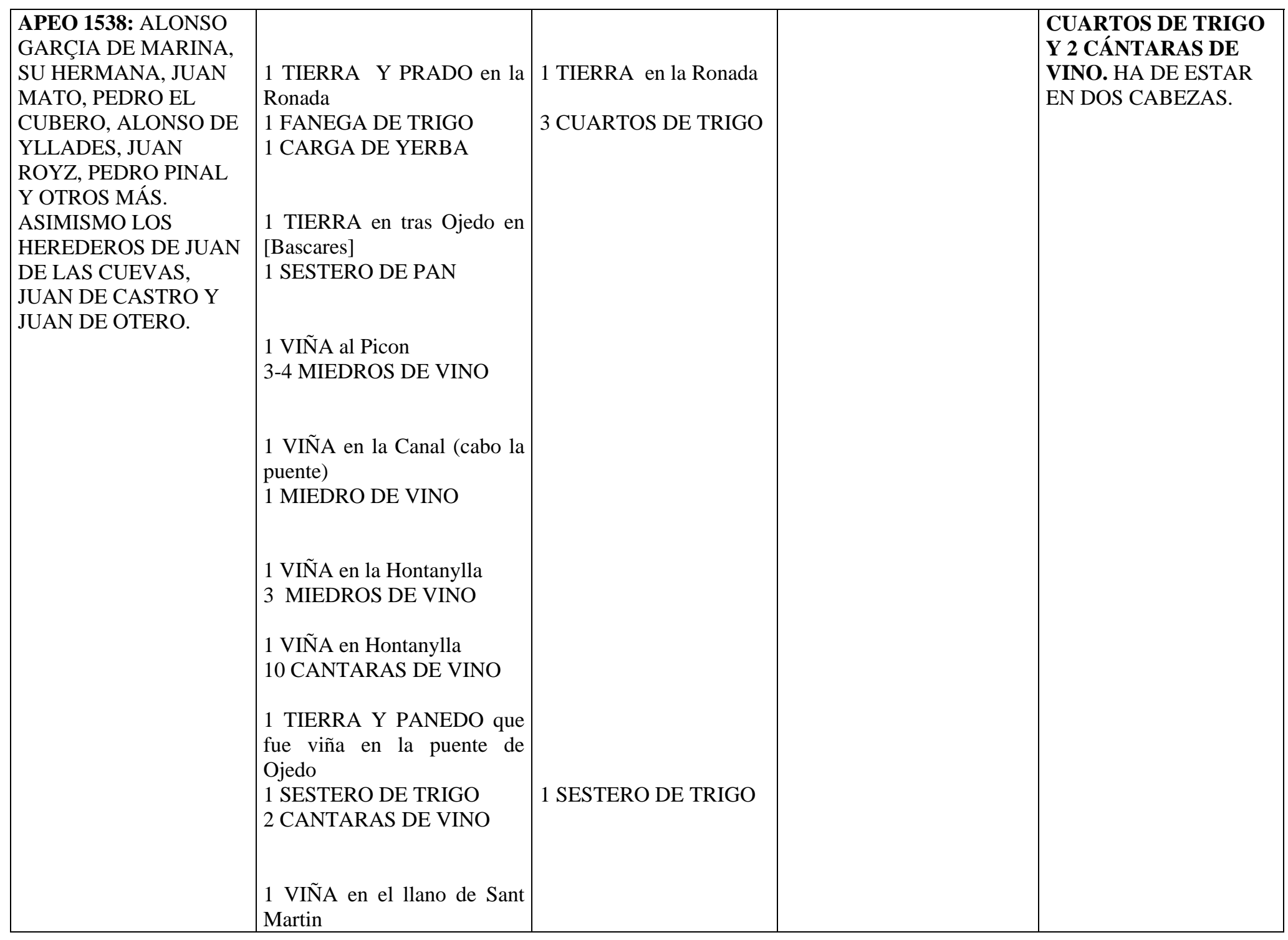




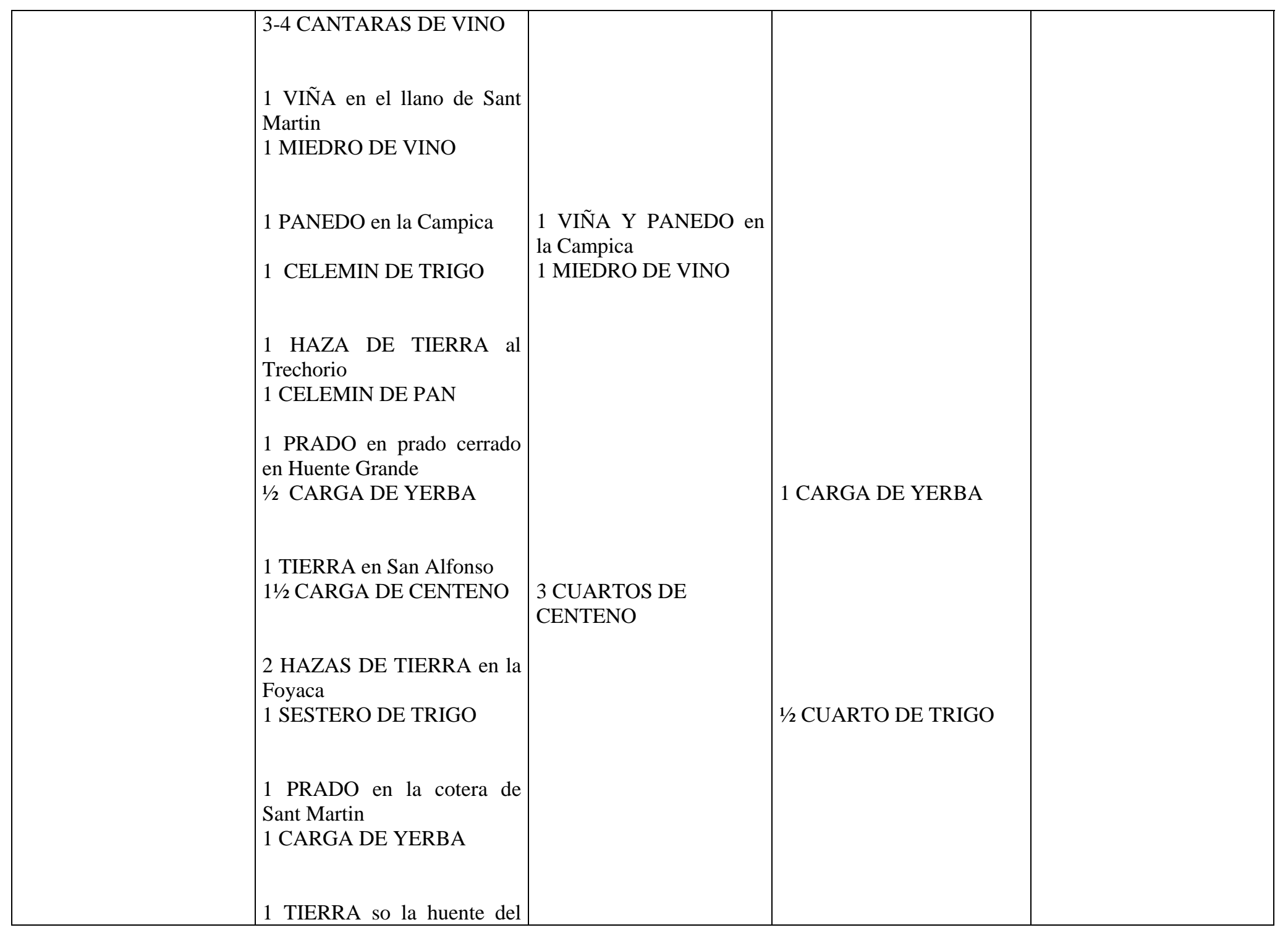




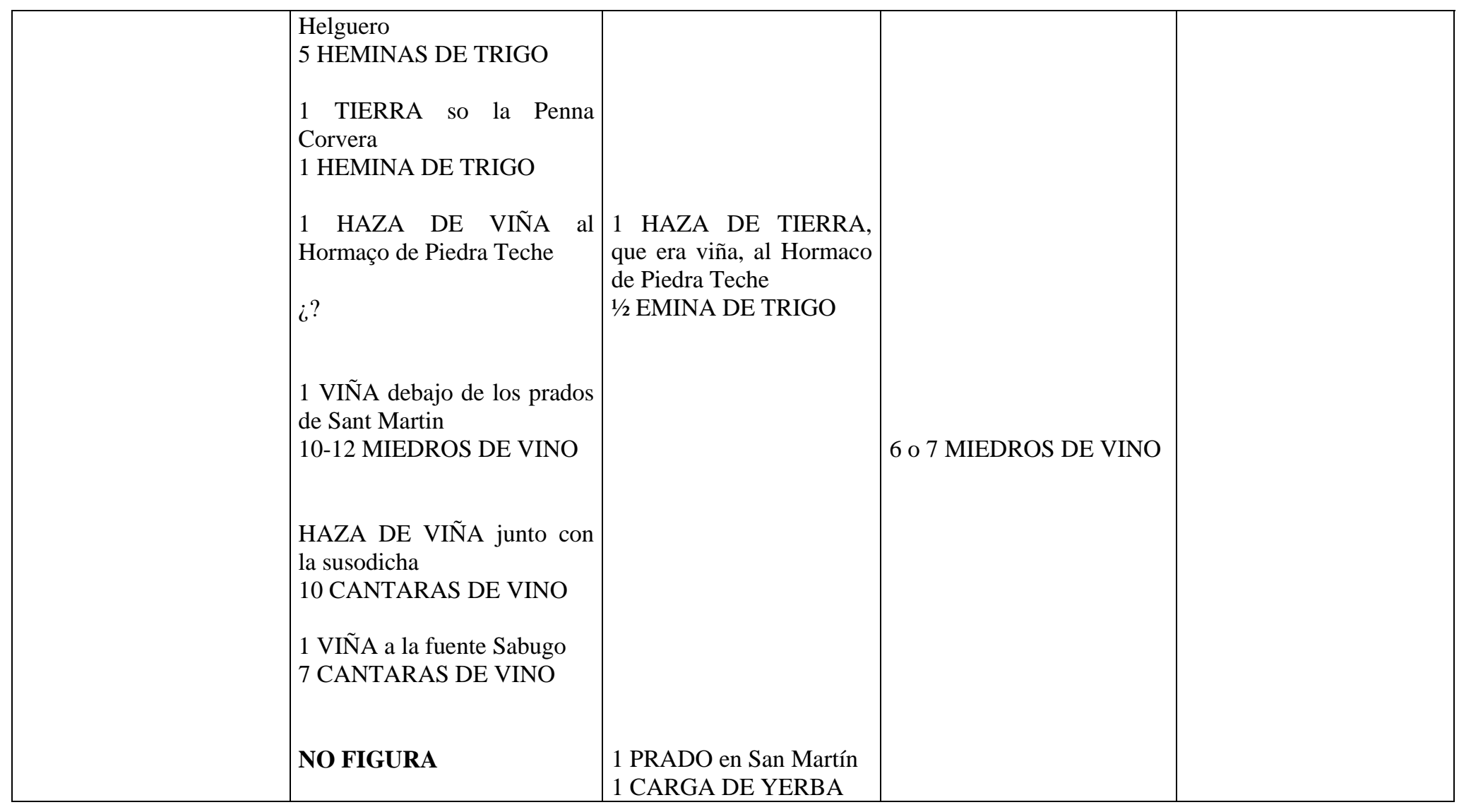

SOLARES EN ALIEZO 


\begin{tabular}{|c|c|c|c|c|}
\hline LUGAR & 1499 & 1515 & 1538 & RENTA \\
\hline $\begin{array}{l}\text { Aldea de ALIEZO } \\
1499 \text { (3) } 1515 \text { y 1538: } \\
\text { JUAN MORAL }\end{array}$ & $\begin{array}{l}1 \text { HUERTA en so casa del } \\
\text { maestro de Aliezo } \\
1 \text { SESTERO DE TRIGO } \\
1 \text { VIÑA so la huerta del } \\
\text { Sennor } \\
3 \text { MIEDROS DE VINO } \\
\text { NO FIGURA } \\
\text { NO FIGURA } \\
\text { NO FIGURA }\end{array}$ & $\begin{array}{l}1 \text { HAZA DE VIÑA en } \\
\text { San Martín } \\
2 \text { CARGAS DE UVAS } \\
1 \text { TIERRA a la cruz de } \\
\text { Santa Maria de Tama } \\
1 \text { SESTERO DE TRIGO } \\
1 \text { VIÑA en Sant Martin } \\
2 \text { CARGAS DE UVAS }\end{array}$ & & $\begin{array}{l}1499 \text { Y 1515: PAGA DE } \\
\text { INFURCION } 3 \text { EMINAS } \\
\text { DE TRIGO Y } 1 / 2 \\
\text { CANTARA DE VINO. } \\
\text { APEO 1538: IDEM y } 1 \\
\text { CANTARA DE VINO } \\
\text { 1515: PAGA DE } \\
\text { INFURCION POR LAS } \\
\text { VIÑAS QUE SE LE } \\
\text { ACRECIENTAN [blanco] }\end{array}$ \\
\hline $\begin{array}{l}\text { Lugar de ALIEZO . Solar } \\
\text { de la Calleja } \\
\text { 1499: (1) Está } \\
\text { derribado. } \\
\text { 1515: MARIA, mujer que } \\
\text { fue de TORIBIO EL } \\
\text { HERRERO, JUAN } \\
\text { MONJE, JUAN RUIZ, } \\
\text { vecinos de Aliezo y dos } \\
\text { hijas del dicho JUAN } \\
\text { MONJE. }\end{array}$ & $\begin{array}{l}1 \text { VIÑA en Río Mayor } \\
2 \text { CANTARAS DE VINO } \\
1 \text { VIÑA en Sant Martin } \\
\text { 2-3 MIEDROS DE VINO } \\
\text { 1 VIÑA en Sant Martin } \\
\text { 4-5 CANTARAS DE VINO } \\
1 \text { VIÑA en el Calar }\end{array}$ & $\begin{array}{l}\text { 2-3 CANTARAS DE } \\
\text { VINO }\end{array}$ & $\begin{array}{l}1 \text { TIERRA en Río Mayor } \\
1 \text { HEMINA DE PAN } \\
1 \text { VIÑA en so Aliezo } \\
3 \text { MIEDROS DE VINO } \\
1 \text { TIERRA en el Calar }\end{array}$ & $\begin{array}{l}\text { 1499 y 1515: SE } \\
\text { INDICA EN EL } \\
\text { SOLAR DE HONDÓN } \\
\text { DE VILLA } \\
\text { APEO 1538: PAGA DE } \\
\text { INFURCION } 6 \text { EMINAS } \\
\text { DE PAN ( } 1 / 2 \text { TRIGO Y } \\
\text { 1/2 CENTENO), } 1 \\
\text { CANTARA DE VINO Y } \\
1 \text { GALLINA. }\end{array}$ \\
\hline
\end{tabular}




\begin{tabular}{|c|c|c|c|c|}
\hline $\begin{array}{l}\text { 1538: MARIA DEL } \\
\text { HERRERO, TORIBIO } \\
\text { EL HERRERO, JUAN } \\
\text { PALACIO y OTROS. }\end{array}$ & $\begin{array}{l}3 \text { MIEDROS DE VINO } \\
\text { estando poblada } \\
1 \text { ERO en los Quadrillos } \\
1 \text { CUARTO DE TRIGO } \\
1 \text { HUERTO en Aliezo } \\
\text { ¿? } \\
2 \text { HAZAS DE VIÑA en el } \\
\text { Calar } \\
3-4 \text { CANTARAS DE VINO } \\
1 \text { TIERRA en la vega, al } \\
\text { nozal de Orejón } \\
1 \text { SESTERO DE TRIGO } \\
2 \text { PANEDOS en el Calar } \\
\text { estan despoblados cabra } \\
1 \text { SESTERO DE PAN } \\
1 \text { VIÑA a la Concha } \\
2 \text { CANTARAS DE VINO }\end{array}$ & $\begin{array}{l}1 \quad \text { TIERRA en los } \\
\text { Quadrillos } \\
1 \text { CUARTO DE TRIGO } \\
2 \text { HAZAS DE TIERRA } \\
\text { en el Calar (era viña) } \\
1 \text { CUARTO DE TRIGO } \\
\text { NO FIGURA }\end{array}$ & $\begin{array}{l}11 \frac{1}{2} \text { CUARTO DE TRIGO } \\
2 \text { SESTEROS DE TRIGO } \\
\text { NO FIGURA }\end{array}$ & $\begin{array}{l}\text { DE ESTE SOLAR } \\
\text { LLEVA LA TERCIA } \\
\text { PARTE ALONSO DE } \\
\text { FONDON DE VILLA Y } \\
\text { ASIMISMO SE DICE EL } \\
\text { SOLAR DE HONDON } \\
\text { DE VILLA (es todo el } \\
\text { solar de la Calleja) }\end{array}$ \\
\hline $\begin{array}{l}\text { ALIEZO. Solar en } \\
\text { Hondon de Villa } \\
\text { 1499 (2) y 1515: } \\
\text { ALONSO, hijo de } \\
\text { TORIBIO DE HONDON } \\
\text { DE VILLA }\end{array}$ & $\begin{array}{l}1 \text { VIÑA so el dicho solar } \\
\text { ¿? } \\
1 \text { TIERRA en el Callar } \\
1 \text { SESTERO DE TRIGO } \\
1 \text { TIERRA a la comporta } \\
1 \text { HEMINA DE TRIGO }\end{array}$ & & NO FIGURA & $\begin{array}{l}\text { PAGAN DE } \\
\text { INFURCION LOS } \\
\text { DICHOS DOS } \\
\text { SOLARES DE LA } \\
\text { CALLEJA Y DE } \\
\text { HONDON DE VILLA } \\
\text { CON SUS PRESTAMOS } \\
6 \text { HEMINAS DE PAN } \\
\text { MITAD TRIGO MITAD }\end{array}$ \\
\hline
\end{tabular}

${ }^{6}$ 1499: AGREGADO AL FINAL DEL PARRAFO LLEVAN LOS DOS SOLARES JUAN DE JUAN RUBIO, JUAN MONGE Y OTROS 


\begin{tabular}{|c|c|c|c|}
\hline & & & $\begin{array}{l}\text { CENTENO, } 1 \\
\text { CANTARA DE VINO Y } \\
1 \text { GALLINA }{ }^{7} .\end{array}$ \\
\hline $\begin{array}{l}\text { ALDEA DEL VALLE } \\
\text { (Cerca de Aliezo) } \\
\text { 1499: (4) NO SE INDICA } \\
\text { 1515: TORIBIO DEL } \\
\text { VALLE } \\
\text { 1538: JUAN DEL } \\
\text { VALLE }\end{array}$ & $\begin{array}{l}1 \text { VIÑA en cabo la huerta del } \\
\text { Señor } \\
4 \text { CANTARAS DE VINO } \\
1 / 2 \text { VIÑA en la huertas del } \\
\text { Señor } \\
1 \text { CANTARA DE VINO } \\
1 \text { TIERRA en so Ojedo en } \\
\text { Matyega } \\
1 \text { HEMINA DE TRIGO } \\
1 \text { VIÑA en tras Ojedo en el } \\
\text { llano Peral } \\
1 \text { CANTARA DE VINO }\end{array}$ & $\begin{array}{l}1 \text { VIÑA en el dicho lugar } \\
\text { de la huerta del Señor } \\
\text { Duque } \\
4 \text { CANTARAS DE VINO } \\
1 \text { VIÑA en tras Ojedo en } \\
\text { el llano Peral } \\
2 \text { CANTARAS DE VINO } \\
\text { (estando plantada) } \\
\text { ESTÁ YERMA }\end{array}$ & $\begin{array}{l}\text { PAGA DE INFURCION } \\
3 \text { EMINAS DE PAN } 1 / 2 \\
\text { TRIGO Y 1/2 CENTENO, } \\
1 / 2 \text { CANTARA DE VINO } \\
\text { Y } 1 \text { GALLINA(en } 1499 \\
\text { esta agre- gada al final del } \\
\text { parrafo) APEO 1538: } \\
\text { IDEM } 1 \text { CANTARA DE } \\
\text { VINO }\end{array}$ \\
\hline
\end{tabular}

\section{SOLARES EN TAMA}

\begin{tabular}{|l|l|l|l|}
\hline LUGAR & \multicolumn{1}{|c|}{1499} & 1515 & 1538 \\
\hline Solar en el barrio de villa & & & RENTA \\
1499: PEDRO EL & 1 HUERTA en so Casa & & PAGA DE INFURCION \\
TARTAMUDO & 3 HEMINAS DE TRIGO & & 1 SESTERO DE TRIGO \\
1515: JUAN & & & 1 CUARTO DE TRIGO, \\
\hline
\end{tabular}

7 (1515) LOS 2/3 LOS LLEVADORES DEL SOLAR DE LA CALLEJA Y EL 1/3 RESTANTE LOS DEL SOLAR DE HONDON DE VILLA 


\begin{tabular}{|c|c|c|c|}
\hline $\begin{array}{l}\text { GUTIERREZ, hijo de } \\
\text { PEDRO GUTTIERREZ, } \\
\text { tartamudo } \\
\text { 1538: ALONSO } \\
\text { GUTIERREZ, clérigo }\end{array}$ & $\begin{array}{l}1 \text { TIERRA en Campanana } \\
1 \text { CUARTO DE TRIGO } \\
1 \text { TIERRA en Campanana } \\
1 \text { CUARTO DE TRIGO }\end{array}$ & & VINO $^{8}$. \\
\hline $\begin{array}{l}\text { Aldea de Tama. Solar de } \\
\text { Juan de la Fuente } \\
\text { 1499: JUAN DE LA } \\
\text { FUENTE y ahora lo lleva } \\
\text { MARÍA, su mujer. } \\
\text { 1515: FERRAN } \\
\text { CAVIDES, PEDRO } \\
\text { CASTAÑEDO, vecinos } \\
\text { de Bedoya y PEDRO } \\
\text { ALEGRE DE TAMA } \\
\text { 1538: No se indica }\end{array}$ & $\begin{array}{l}1 \text { VIÑA en termino de } \\
\text { Campanana } \\
3 \text { MIEDROS DE VINO } \\
\text { estando bien poblada } \\
1 \text { TIERRA en Campanana } \\
6 \text { HEMINAS DE TRIGO } \\
1 \text { TIERRA en Campanana } \\
1 \text { SESTERO DE TRIGO } \\
1 \text { VIÑA en el rio Viñon } \\
3 \text { MIEDROS DE VINO } \\
1 \text { TIERRA en el termino de } \\
\text { Campanana } \\
1 \text { SESTERO DE PAN }\end{array}$ & $\begin{array}{l}\dot{¿} ? \\
6 \text { CARGAS DE UVAS } \\
3 \text { HEMINAS DE TRIGO }\end{array}$ & $\begin{array}{l}\text { PAGA DE INFURCION } \\
1 \text { CUARTO DE TRIGO } \\
\text { Y 1 CANTARA DE } \\
\text { VINO } \\
\text { APEO 1538: } \\
\text { Aparentemente figura un } \\
\text { [blanco] al que se le } \\
\text { agregó } 6 \text { HEMINAS DE } \\
\text { TRIGO }\end{array}$ \\
\hline $\begin{array}{l}\text { ALDEA DE TAMA. } \\
\text { Solar en el Barrio de suso } \\
\text { 1499: Herederos de } \\
\text { JUAN DE TAMA y } \\
\text { JUAN GRANDE DE } \\
\text { OJEDO } \\
\text { 1515: TORIBIO DE }\end{array}$ & $\begin{array}{l}1 \text { TIERRA en las vallejas de } \\
\text { Tama } \\
1 \text { 1/2 HEMINAS DE TRIGO } \\
1 \text { TIERRA a las Cortynas de } \\
\text { Tama }\end{array}$ & & $\begin{array}{l}\text { Este dicho solar solía } \\
\text { pagar de infurcion } 3 \\
\text { PANES Y } 1 \text { GALLINA. } \\
\text { LA } 1 / 2 \text { PAGA DE } \\
\text { INFURCION } 1 / 2 \\
\text { GALLINA Y } 1 \text { 1 } 12 \text { PAN }^{9} .\end{array}$ \\
\hline
\end{tabular}
${ }^{8}$ HA DE QUEDAR EN 1 HEREDERO Y NO MAS. “El SEÑOR PRIOR y su mandado PUEDE ENTRAR Y TOMAR LAS PRENDAS A LOS QUE
LLEVAN LOS SOLARES y pagan todas las costas que se hicieren en la cobranza de la infurcion de los solares”

${ }^{9}$ SE VENDIO LA MITAD DEL SOLAR A HEREDEROS DE ALONSO DUQUE Y LA 1/2 QUEDO CON EL DICHO MONASTERIO 
JUAN DE TAMA DE

VIÑON. Solía llevar Juan

Gutierrez de Hogedo

1538: NO FIGURA

SOLARES EN ARMAÑO

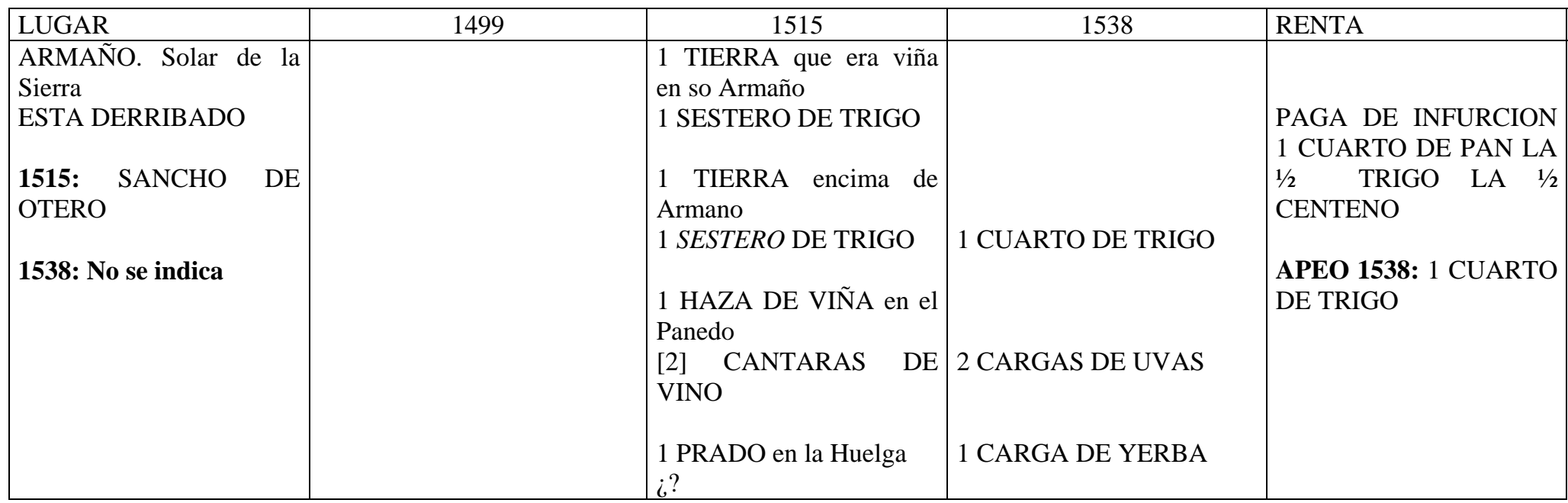

\section{SOLARES EN FRAMA}

\begin{tabular}{|l|l|l|l|}
\hline \multicolumn{1}{|c|}{ TENENTE } & \multicolumn{1}{|c|}{1499} & 1515 & 1538 \\
\hline $\begin{array}{l}\text { (1515) } 1 \text { SOLAR Y } 1 / 2 \\
\text { El solar de la Puente. }\end{array}$ & $\begin{array}{l}\text { 1 VIÑA en la Cabanilla } \\
\text { 4-5 MIEDROS DE VINO }\end{array}$ & $\begin{array}{l}\text { 1 TIERRA en la Cabanilla } \\
\text { 1 FANEGA DE TRIGO }\end{array}$ & \\
1515: DIEGO DE LA & 1 TIERRA en el Hoyo de & & \\
\hline
\end{tabular}




\begin{tabular}{|c|c|c|c|c|}
\hline $\begin{array}{l}\text { VEGA, hijo de Fernando } \\
\text { de La Vega y OTROS } \\
\text { 1538: HERNANDO DE } \\
\text { SALCEDA, JUAN DE } \\
\text { NAREZO Y ALONSO } \\
\text { VEDOYA } \\
\text { (lo de Alonso Vedoya } \\
\text { ESTA VACO) }\end{array}$ & $\begin{array}{l}\text { Santa Cruz } \\
3 \text { HEMINAS DE PAN } \\
1 \text { TIERRA en Quintana } \\
1 \text { SESTERO DE TRIGO } \\
1 \text { TIERRA en el Hoyo } \\
1 \text { FANEGA DE PAN } \\
1 \text { PANEDO en tras Palaçio } \\
1 \text { CANTARA DE VINO } \\
\text { plantándose } \\
1 \text { VIÑA en Cerezalín } \\
1 \text { CANTARA DE VINO } \\
1 \text { TIERRA en Caldehierro } \\
3 \text { HEMINAS DE TRIGO } \\
1 \text { PANEDO en tras Palaçio } \\
1 \text { 1/2 HEMINA DE PAN } \\
1 \text { TIERRA en tras Hojedo } \\
\text { ¿? } \\
1 \text { haza de VIÑA en Cereçalin. } \\
2-3 \text { CANTARAS DE VINO } \\
\text { ¿? }\end{array}$ & 2 CANTARAS DE VINO & $\begin{array}{l}1 \text { CUARTO DE TRIGO } \\
1 \text { VINA en Pedredo } \\
\text { CANTARA y } 1 / 2 \quad \text { DE } \\
\text { VINO } \\
\text { NO FIGURA } \\
\text { NO FIGURA } \\
\text { CUARTOS DE PAN }\end{array}$ & $\begin{array}{l}\text { PAGA DE INFURCION } \\
9 \text { HEMINAS DE PAN (1/2 } \\
\text { TRIGO } 1 / 2 \text { CENTENO) } \\
1 \quad 1 / 2 \text { CANTARA DE } \\
\text { VINO Y } 3 \text { BLANCAS } \\
\text { VIEJAS DE SUELDO. } \\
\text { APEO 1538: IDEM y } 4 \\
\text { MARAVEDIES Y } 1 / 2 \text { EN } \\
\text { MONEDA. }\end{array}$ \\
\hline $\begin{array}{l}\text { SOLAR DEL PRADO } \\
\text { 1515: DIEGO DE LA } \\
\text { VEGA E HIJOS DE } \\
\text { TORIBIO DEL ABAD }\end{array}$ & $\begin{array}{l}1 \text { TIERRA en Navajos } \\
3 \text { CUARTOS DE PAN } \\
1 \quad \text { TIERRA en Navajos } \\
\text { (debajo de la otra) } \\
1 \text { CUARTO DE TRIGO }\end{array}$ & & & \\
\hline
\end{tabular}




\begin{tabular}{|c|c|c|c|c|}
\hline $\begin{array}{lll}\text { 1538: } & \text { ALONSO } & \text { DEL } \\
\text { ABAD } & & \\
\end{array}$ & $\begin{array}{l}\text { NO FIGURA } \\
1 \text { TIERRA en la Erçina } \\
5 \text { HEMINAS DE TRIGO } \\
1 \text { VIÑA en la Vega de Frama } \\
4 \text { MIEDROS DE VINO } \\
1 \text { VIÑA en la Redonda } \\
2 \text { MIEDROS DE VINO } \\
1 \text { VIÑA en Molin Gayon } \\
3 \text { MIEDROS DE VINO } \\
1 \text { TIERRA en la vega de } \\
\text { Frama al portyllo } \\
3 \text { HEMINAS DE TRIGO } \\
1 \text { TIERRA al llano de } \\
\text { Nabajos } \\
3 \text { HEMINAS DE TRIGO } \\
1 \text { TIERRA en el valle de } \\
\text { Lubayo } \\
1 \text { FANEGA DE PAN } \\
1 \text { TIERRA en Val de } \\
\text { Quintana } \\
1 \text { CUARTO DE PAN }\end{array}$ & $\begin{array}{l}1 \text { VIÑA en el llano de } \\
\text { Pedredo } \\
1 \text { CARGA DE UVAS } \\
8 \text { HEMINAS DE TRIGO }\end{array}$ & 更 & $\begin{array}{l}\text { PAGA DE INFURCION } \\
3 \text { CUARTOS DE PAN } \\
\text { (1/2 TRIGO 1/2 CENTENO) } \\
2 \text { CANTARAS DE VINO } \\
\text { Y } 2 \text { MARAVEDIES DE } \\
\text { SUELDO EN CADA } \\
\text { AÑO. } \\
\text { APEO 1538: NO } \\
\text { FIGURAN LOS } \\
\text { MARAVEDIES }\end{array}$ \\
\hline $\begin{array}{l}\text { SOLAR DE MARTIN } \\
\text { GARCÍA en el Barrio de } \\
\text { San Juan }\end{array}$ & $\begin{array}{l}1 \text { VIÑA en Trigales } \\
5 \text { a } 6 \text { MIEDROS DE VINO } \\
1 \text { VIÑA en Cederne } \\
\text { 2-3 CANTARAS DE VINO }\end{array}$ & 1 MIEDRO DE VINO & 3 CANTARAS DE VINO & $\begin{array}{l}\text { PAGA DE INFURCION } \\
6 \text { HEMINAS DE PAN } \\
(1 / 2 \text { TRIGO } 1 / 2\end{array}$ \\
\hline
\end{tabular}




\begin{tabular}{|c|c|c|c|c|}
\hline $\begin{array}{lr}\text { 1515: ANTÓN } & \text { DE } \\
\text { CILLANUEVA Y } & \text { SUS } \\
\text { HEREDEROS } & \\
\text { 1538: JUAN } & \text { DE } \\
\text { CILLANUEVA, } \\
\text { HERNANDO } \\
\text { CILLANUEVA, JUAN } \\
\text { DE NAREZO Y Y } \\
\text { TORIBIO HERRERO, } \\
\text { vecinos de Frama. }\end{array}$ & $\begin{array}{l}1 \text { TIERRA en Sant Bartolomé } \\
1 \text { HEMINA DE TRIGO } \\
1 \text { TIERRA en el llano } \\
\text { Redondo } \\
1 \text { CUARTO DE PAN } \\
1 \text { TIERRA en Cereçalin } \\
1 \text { HEMINA DE PAN } \\
1 \text { HUERTO, TIERRA Y } \\
\text { VIÑA en Cabarieço } \\
1 \text { CANTARA DE VINO } \\
1 \text { HEMINA DE TRIGO } \\
1 \text { VIÑA en Trigales } \\
2 \text { CANTARAS DE VINO } \\
1 \text { VIÑA al ponton de Viedo } \\
\text { que llevan los herederos del } \\
\text { cavallero. } \\
2 \text { CANTARAS DE VINO }\end{array}$ & (cavándola y labrándola) & $\begin{array}{l}1 \text { HEMINA DE TRIGO } \\
1 \text { VIÑA en Çereçalin } \\
1 \text { CARGA DE UVAS } \\
1 \text { TIERRA que era viña } \\
1 \text { HEMINA DE PAN }\end{array}$ & $\begin{array}{l}\text { CENTENO) } 1 \\
\text { CANTARA DE VINO Y } \\
1 \text { MARAVEDI DE } \\
\text { SUELDO. }\end{array}$ \\
\hline
\end{tabular}

SOLARES EN CAMBARCO

\begin{tabular}{|lr|l|c|l|l|}
\hline LUGAR & & \multicolumn{1}{|c|}{1499} & 1515 & 1538 \\
\hline \begin{tabular}{ll|l} 
SOLAR \\
OVESAS
\end{tabular} & DE & LAS & 1 VIÑA en Canaliço & & RENTA \\
1538: & & 2 CANTARAS DE VINO & & $\begin{array}{l}\text { 2 MIEDROS DE VINO } \\
\text { (estando bien labrada) }\end{array}$ \\
\hline
\end{tabular}




\begin{tabular}{|c|c|c|c|c|}
\hline $\begin{array}{l}\text { DERRIBADO } \\
\text { ALONSO DE LAS } \\
\text { OVESAS Y GUTIERRE } \\
\text { DE LAS OVESAS SU } \\
\text { HEREDERO } \\
\text { 1538: GUTIERRE DE } \\
\text { LAS OVESAS }\end{array}$ & $\begin{array}{l}1 \text { VIÑA en la riega del Gato } \\
2 \text { CANTARAS DE VINO } \\
\text { (labrándose) } \\
1 \text { FAÇA DE VIÑA en las } \\
\text { Casas } \\
2 \text { CANTARAS DE VINO } \\
1 \text { VIÑA en el Carrascal } \\
3-4-C A N T A R A S \text { DE VINO } \\
1 \text { TIERRA en Haza Modin } \\
1 \text { HEMINA DE TRIGO o } \\
\text { más } \\
1 \text { TIERRA en el Huerto de } \\
\text { las Ovesas } \\
1 \text { HEMINA DE TRIGO } \\
1 / 2 \text { HUERTO adelante del } \\
\text { dicho solar } \\
\dot{¿} ? \\
1 / 2 \text { ERA que esta sobre el } \\
\text { dicho solar }\end{array}$ & 2 HEMINAS DE TRIGO & $\begin{array}{l}\text { Es PANEDO } \\
\text { 3-4 CANTARAS DE } \\
\text { VINO (labrándose) } \\
\text { 3-4 CANTARAS DE VINO } \\
\text { (si se labrase) } \\
1 \text { SESTERO DE TRIGO } \\
\end{array}$ & $\begin{array}{c}\text { PAGA DE INFURCION } \\
6 \text { HEMINAS DE PAN } \\
(1 / 2 \text { TRIGO } 1 / 2 \\
\text { CEVADA })\end{array}$ \\
\hline $\begin{array}{l}\text { Solar de Palacio } \\
\text { 1515: GOMEZ DE LA } \\
\text { CARRADA } \\
\text { 1538: TORIBIO GOMEZ } \\
\text { Y SU MADRE }\end{array}$ & $\begin{array}{l}1 \text { VIÑA en Canalizo } \\
1 \text { MIEDRO DE VINO } \\
1 \text { VIÑA en la hera del Gato } \\
3 \text {-4 CANTARAS DE VINO } \\
\text { (Estando guisada ) } \\
1 \text { HAZA DE VIÑA en las } \\
\text { Casas }\end{array}$ & & & $\begin{array}{l}\text { PAGA DE INFURCION } \\
6 \text { HEMINAS DE PAN } \\
(1 / 2 \text { TRIGO } 1 / 2 \\
\text { CEVADA })\end{array}$ \\
\hline
\end{tabular}




\begin{tabular}{|c|c|c|c|c|}
\hline & $\begin{array}{l}2 \text { CANTARAS DE VINO } \\
1 \text { HAZA DE VIÑA en las } \\
\text { Casas que se decía el } \\
\text { Carrascal } \\
4 \text { CANTARAS DE VINO } \\
1 \text { TIERRA en Haça Modin } \\
1 \text { EMINA DE PAN } \\
1 \text { TIERRA en los Huertos de } \\
\text { las Ovesas } \\
1 \text { SESTERO DE TRIGO } \\
1 \text { HUERTO delante del solar } \\
\text { de las Ovesas } \\
\text { ¿? } \\
1 \text { TIERRA a la Portylla } \\
1 / 2 \text { HEMINA DE TRIGO } \\
1 / 2 \text { ERA sobre el solar de las } \\
\text { Ovesas }\end{array}$ & 西 & $\begin{array}{l}\text { CANTARAS DE VINO } \\
\text { si se planta. ESTA YERMA } \\
1 \text { HEMINA DE PAN } \\
1 \text { SESTERO DE TRIGO }\end{array}$ & $\begin{array}{l}\text { APEO 1538: } \\
\text { ENTIENDASE QUE } \\
\text { ESTE SOLAR QUE SE } \\
\text { HALLA EN EL APEO } \\
\text { VIEJO LE QUITARON } \\
\text { DE LAS } 6 \text { EMINAS } 1 \\
\text { SESTERO. } \\
\text { HA DE PAGAR DE } \\
\text { AQUÍ EN ADELANTE } \\
\text { UN CUARTO DE } \\
\text { TRIGO }\end{array}$ \\
\hline $\begin{array}{l}\text { 1538: Solar de la Cortina. } \\
\text { 1515: TORIBIO DE LA } \\
\text { CORTINA, hijo de Juan } \\
\text { Martín de la Cortina. } \\
\text { 1538: TORIBIO DE LA } \\
\text { CORTINA }\end{array}$ & $\begin{array}{l}1 \text { HUERTO en Cambarço } \\
\text { sobre la calleja. } \\
1 \text { SESTERO DE PAN }\end{array}$ & & 1 HEMINA DE PAN & $\begin{array}{l}\text { PAGA DE INFURCION } \\
1 \text { CUARTO DE } \\
\text { CEVADA. }\end{array}$ \\
\hline
\end{tabular}




\section{SOLARES EN CAHECHO}

\begin{tabular}{|c|c|c|c|c|}
\hline TENENTE & 1499 & 1515 & 1538 & RENTA \\
\hline $\begin{array}{l}\text { SOLAR DE PUMAR } \\
\text { 1515: HIJOS DE PEDRO } \\
\text { GARCÍA DE CAHECHO } \\
\text { 1538: PEDRO GARCÍA } \\
\text { DE CAHECHO }\end{array}$ & $\begin{array}{l}1 \text { VIÑA en Posadorio } \\
1 \text { MIEDRO DE VINO } \\
1 \text { TIERRA en Llan del Sayon } \\
1 \text { CUARTO DE CENTENO } \\
1 \text { TIERRA en la Padrenga } \\
1 \text { CUARTO DE PAN } \\
1 \text { TIERRA en Carnejo } \\
6 \text { HEMINAS DE PAN } \\
1 \text { PRADO en la Venera } \\
1 \text { CARGA DE YERBA } \\
1 \text { TIERRA en las fuentes } \\
1 \text { HEMINA DE PAN } \\
1 \text { TIERRA en sobre Palaçio } \\
\text { ¿? } \\
1 \text { TIERRA en Valle } \\
1 \text { FANEGA DE PAN } \\
1 \text { TIERRA en Canbrezo } \\
1 \text { SESTERO DE TRIGO } \\
1 \text { TIERRA en Fenogeda } \\
1 \text { SESTERO DE CENTENO }\end{array}$ & ¿? & $\begin{array}{l}\text { ¿? } \\
\text { Esta YERMA } \\
1 \text { CUARTO DE PAN } \\
1 \text { SESTERO DE PAN }\end{array}$ & $\begin{array}{l}\text { PAGA DE INFURCION } \\
2 \text { CUARTOS DE TRIGO }\end{array}$ \\
\hline
\end{tabular}




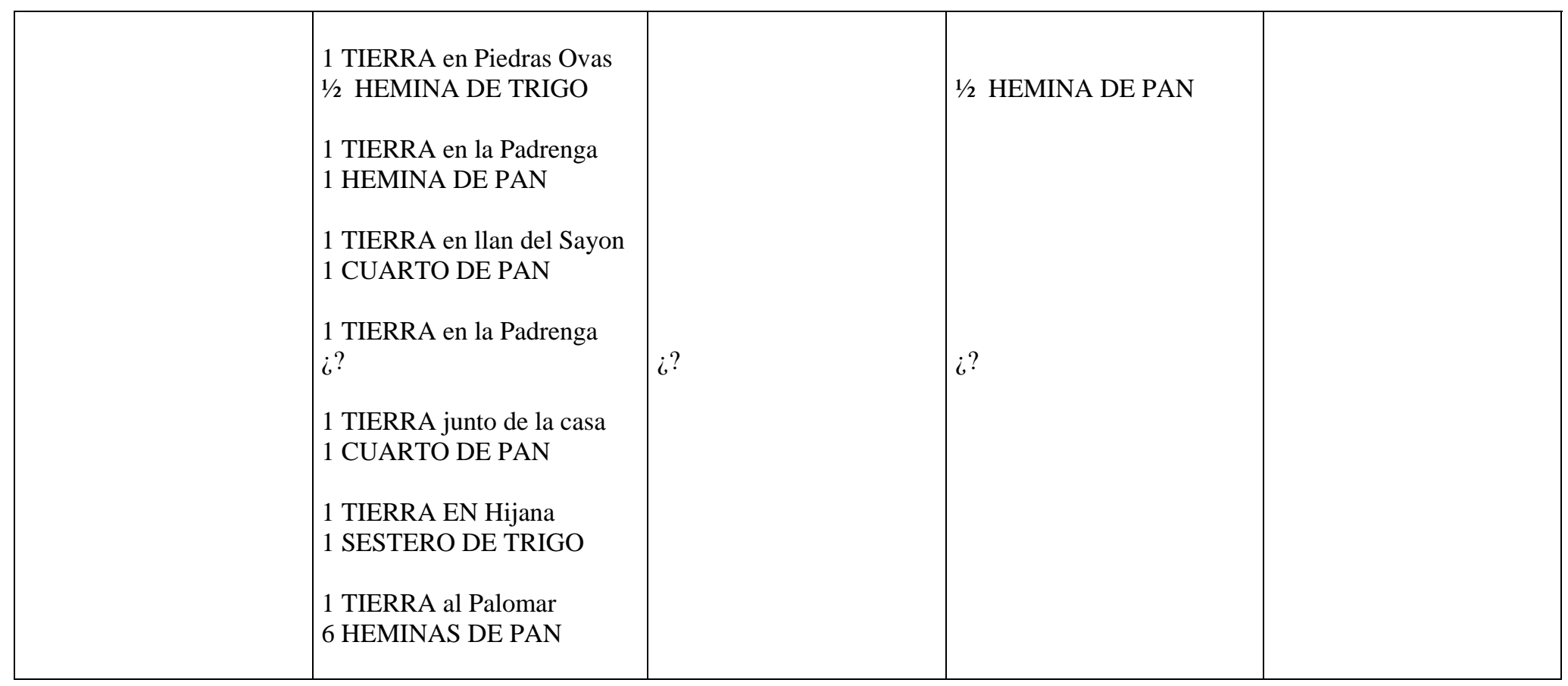




\section{VIÑAS Y HEREDADES A TERCIO EN SANTIBAÑEZ}

\begin{tabular}{|c|c|c|c|c|}
\hline LUGAR & 1499 & 1515 & 1538 & RENTA \\
\hline $\begin{array}{l}\text { 1499: PEDRO GÓMEZ DE } \\
\text { LON, JUAN DE } \\
\text { FERRANDO Y OTROS } \\
\text { MUCHOS } \\
\text { 1515: PEDRO GOMEZ DE } \\
\text { LON, JUAN BLANCO DE } \\
\text { VALDEON Y OTROS } \\
\text { 1538: JUAN GOMEZ DE } \\
\text { LON, PEDRO DE } \\
\text { BEARES Y JUAN EL } \\
\text { HERRERO DE BEARES. }\end{array}$ & $\begin{array}{l}1 \text { VIÑA en so Congarna } \\
\text { que se dice de los Canines } \\
4 \text {-5 MIEDROS DE VINO }\end{array}$ & 5 MIEDROS DE VINO & $\dot{d} ?$ & LLEVAN A CUARTO. \\
\hline $\begin{array}{l}\text { 1499: NO FIGURA } \\
\text { 1515: DIEGO DE BARÓ, } \\
\text { merino. } \\
\text { 1538: TORIBIO DE } \\
\text { CONGARNA }\end{array}$ & & $\begin{array}{l}1 \text { VIÑA que se dice la } \\
\text { Haza Redonda } \\
10 \text { CANTARAS DE } \\
\text { VINO }\end{array}$ & $\dot{d} ?$ & POR TERCIO. \\
\hline $\begin{array}{l}\text { 1499: NO FIGURA } \\
\text { 1515: ALONSO DE } \\
\text { OCEÑO } \\
\text { 1538: NO FIGURA }\end{array}$ & & $\begin{array}{l}1 \text { VIÑA en la Linera } \\
5 \text { MIEDROS DE VINO }\end{array}$ & & $\begin{array}{l}\text { ES DE TERCIO. } \\
\text { En el margen: Trocose } \\
\text { por el molino de Overán, } \\
\text { es heredad. }\end{array}$ \\
\hline $\begin{array}{l}\text { 1499: NO FIGURA } \\
\text { 1515: GARCÍA DE } \\
\text { TECEÑO } \\
\text { 1538: PEDRO } \\
\text { GONZÁLEZ DE } \\
\text { CONGARNA }\end{array}$ & & $\begin{array}{l}1 \text { VIÑA en la Linera } \\
2 \text { MIEDROS DE VINO }\end{array}$ & 3 o 4 CARGAS DE UVAS & POR TERCIO \\
\hline
\end{tabular}




\begin{tabular}{|c|c|c|c|c|}
\hline $\begin{array}{l}\text { 1499: GARCÍA DE LA } \\
\text { FRECHA } \\
\text { 1515: GARCÍA ALONSO } \\
\text { DE LA FRECHA } \\
\text { 1538: HEREDEROS DE } \\
\text { GARCÍA ALONSO DE LA } \\
\text { FRECHA }\end{array}$ & $\begin{array}{l}1 \text { VIÑA en la Requexada de } \\
\text { Mus } \\
2 \text { CANTARAS DE VINO }\end{array}$ & & $\dot{d} ?$ & ES DE TERCIO. \\
\hline $\begin{array}{l}\text { 1499: JUAN DE BARÓ } \\
\text { 1515: TORIBIO GARCÍA } \\
\text { DE CAMALEÑO } \\
\text { 1538: NO FIGURA }\end{array}$ & $\begin{array}{l}1 \text { VIÑA en Mus } \\
2 \text { MIEDROS DE VINO }\end{array}$ & & & \\
\hline $\begin{array}{l}\text { 1499: JUAN DE BARÓ } \\
\text { 1515: ELVIRA GOMEZ } \\
\text { DE TURIENO } \\
\text { 1538: NO FIGURA }\end{array}$ & $\begin{array}{l}1 \text { VIÑA en Mus } \\
\text { ¿? }\end{array}$ & $\begin{array}{l}10 \text { CANTARAS DE } \\
\text { VINO }\end{array}$ & & $\begin{array}{l}\text { POR CUARTO. } \\
\text { En el margen: Trocose } \\
\text { esta viña por la de } \\
\text { Campejón. }\end{array}$ \\
\hline $\begin{array}{l}\text { 1499: NO FIGURA } \\
\text { 1515: DIEGO DE BARÓ, } \\
\text { merino. } \\
\text { 1538: PEDRO } \\
\text { GONZALEZ DE } \\
\text { CONGARNA. }\end{array}$ & & $\begin{array}{l}1 \text { VIÑA en Mus } \\
1 \text { CANTARA DE VINO }\end{array}$ & 1 CARGA DE UVAS & POR TERCIO. \\
\hline $\begin{array}{l}\text { 1499: JUAN DE LA } \\
\text { POSADA } \\
\text { 1515: GONZALO } \\
\text { GUZMÁN } \\
\text { 1538: GARCI PONCE DE } \\
\text { TURIENO }\end{array}$ & $\begin{array}{l}1 \text { VIÑA en el Rexo } \\
1 \text { MIEDRO DE VINO }\end{array}$ & & & POR TERCIO. \\
\hline
\end{tabular}




\begin{tabular}{|c|c|c|c|c|}
\hline 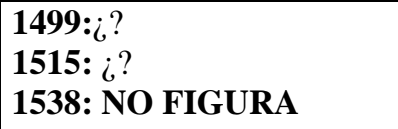 & $\begin{array}{l}1 \text { PANEDO en la Dornilla } \\
1 \text { SESTERO DE PAN }\end{array}$ & & & \\
\hline $\begin{array}{l}\text { 1499: NO FIGURA } \\
\text { 1515: ALONSO } \\
\text { VALLEJO, FERRANDO } \\
\text { DE MESAYNA, GARCÍA } \\
\text { DE MOLLEDA, PEDRO } \\
\text { DE LLANO, PEDRO DE } \\
\text { PIASCA, JUAN, hijo de } \\
\text { PEDRO VALLEJO, } \\
\text { ALONSO DE OCEÑO y } \\
\text { JUAN DE LA CALLEJA } \\
\text { 1538: LA LLEVAN } \\
\text { CIERTOS VECINOS DEL } \\
\text { CONCEJO. }\end{array}$ & & $\begin{array}{l}1 \text { VIÑA en Canpejon } \\
\dot{¿} ?\end{array}$ & $\dot{¿} ?$ & $\begin{array}{l}\text { ES A TERCIO. SE DIO } \\
\text { POR } 30 \text { AÑOS. SALE EL } \\
\text { AÑO DE } 1533 .\end{array}$ \\
\hline $\begin{array}{l}\text { 1499: NO FIGURA } \\
\text { 1515: JUAN DE BARÓ } \\
\text { 1538: NO FIGURA }\end{array}$ & & $\begin{array}{l}1 \text { VIÑA en la Molina } \\
1 \text { MIEDRO DE VINO }\end{array}$ & & ES DE TERCIO. \\
\hline $\begin{array}{l}\text { 1499: JUAN DE LA } \\
\text { POSADA } \\
\text { 1515: MARIA DE LA } \\
\text { POSADA } \\
\text { 1538: NO FIGURA }\end{array}$ & $\begin{array}{l}1 \text { HAZA DE VIÑA en La } \\
\text { Molina } \\
1 \text { PANEDO } \\
1 \text { MIEDRO DE VINO } \\
\text { 3-4 CÁNTARAS DE VINO }\end{array}$ & $\begin{array}{l}1 \text { MIEDRO DE VINO } \\
1 \text { MIEDRO DE VINO } \\
\text { (Plantado) }\end{array}$ & & ES DE CUARTO. \\
\hline $\begin{array}{l}\text { 1499: GARCÍA DE } \\
\text { OTERO, PEDRO DE } \\
\text { OTERO, GONZALO DE } \\
\text { BARÓ, merino. }\end{array}$ & $\begin{array}{l}1 \text { VIÑA en Llan de Otero } \\
\text { 3-4 MIEDROS DE VINO }\end{array}$ & & & $\begin{array}{l}\text { LA LLEVAN } \quad \text { POR } \\
\text { TERCIO. }\end{array}$ \\
\hline
\end{tabular}




\begin{tabular}{|c|c|c|c|c|}
\hline $\begin{array}{l}\text { 1515: JUAN GARCÍA DE } \\
\text { LA CALLEJA, TORIBIO } \\
\text { DE PEDRO DE OTERO, } \\
\text { RODRIGO DE PEDRO DE } \\
\text { OTERO E HIJOS DE } \\
\text { GONZALO DE BARÓ. } \\
\text { 1538: JUAN GARCÍA DE } \\
\text { LA CALLEJA, DIEGO DE } \\
\text { BARÓ, ALONSO, su hijo, } \\
\text { JUAN DE OTERO E INÉS } \\
\text { GONZALEZ DE } \\
\text { TURIENO. }\end{array}$ & & & & \\
\hline $\begin{array}{l}\text { 1499: DIEGO DE BARÓ, } \\
\text { JUAN DE LA CALLEJA Y } \\
\text { GARCÍA DE ARENAS } \\
\text { 1515: DIEGO DE BARÓ, } \\
\text { JUAN DE LA CALLEJA Y } \\
\text { GARCÍA DE MOLLEDA } \\
\text { 1538: PEDRO DE LLANO, } \\
\text { PEDRO DE PIASCA, } \\
\text { PEDRO DE TREVIA Y } \\
\text { JUAN DE LA ERMIDA. }\end{array}$ & $\begin{array}{l}\text { 1 VIÑA en la Cabanna } \\
\text { 6-7 MIEDROS DE VINO }\end{array}$ & & & DE TERCIO. \\
\hline $\begin{array}{l}\text { 1499: ALONSO DEL } \\
\text { CALVO Y TORIBIO, su } \\
\text { heredero. } \\
\text { 1515: PEDRO DEL } \\
\text { LLANO Y ALONSO DEL } \\
\text { CALVO } \\
\text { 1538: NO FIGURA }\end{array}$ & $\begin{array}{l}1 \text { VIÑA en la Cabaña } \\
3 \text { MIEDROS DE VINO }\end{array}$ & & & ES DE TERCIO. \\
\hline $\begin{array}{l}\text { 1499: HEREDEROS DE } \\
\text { PEDRO VALLEJO Y DE }\end{array}$ & $\begin{array}{l}1 \text { VIÑA en al Valladar } \\
10 \text { MIEDROS DE VINO }\end{array}$ & 6-7 MIEDROS DE VINO & 10 MIEDROS DE VINO & DE TERCIO. \\
\hline
\end{tabular}




\begin{tabular}{|c|c|c|c|c|}
\hline $\begin{array}{l}\text { JUAN DE LA CALLEJA } \\
\text { 1515: MARTIN DE JUAN } \\
\text { DE DIEGO, TORIBIO } \\
\text { ESPINEL, PEDRO DE } \\
\text { CEREBANES Y } \\
\text { GONZALO DE MENCIA } \\
\text { 1538: MARTIN DE } \\
\text { BARÓ, PEDRO DE LA } \\
\text { HERREN, PEDRO DE } \\
\text { CEREBANES, JUAN DE } \\
\text { LA PUENTE Y JUAN DE } \\
\text { LA FUENTE, el mozo. }\end{array}$ & & & & \\
\hline $\begin{array}{l}\text { 1499: ALONSO DE } \\
\text { MENCIA, GARCÍA DE } \\
\text { NORIEGA, JUANA DE } \\
\text { PEDRO, GONZALO DE } \\
\text { BARÓ, JUAN DE LA } \\
\text { POSADA, HEREDEROS } \\
\text { DE RODRIGO DE } \\
\text { MIESES, HEREDEROS } \\
\text { DE JUAN DE } \\
\text { FLORANES, JUAN DE } \\
\text { BARÓ, GARCÍA DE } \\
\text { OTERO, TERESA, la } \\
\text { casera, FERNANDO DE } \\
\text { BARÓ, GARCÍA DE } \\
\text { COLLAZOS, ALONSO } \\
\text { DEL CALVO, JUAN DE } \\
\text { TRECEÑO, ALONSO DE } \\
\text { LA CALLEJA, GONZALO } \\
\text { DE GUZMÁN, GONZALO } \\
\text { SÁNCHEZ, JUAN } \\
\text { HERRERO Y OTROS } \\
\text { MUCHOS. } \\
\text { 1515: GONZALO }\end{array}$ & $\begin{array}{l}1 \text { VIÑA en Viña Mayor } \\
35 \text { MIEDROS DE VINO }\end{array}$ & 30 MIEDROS DE VINO & $\dot{¿} ?$ & POR TERCIO. \\
\hline
\end{tabular}




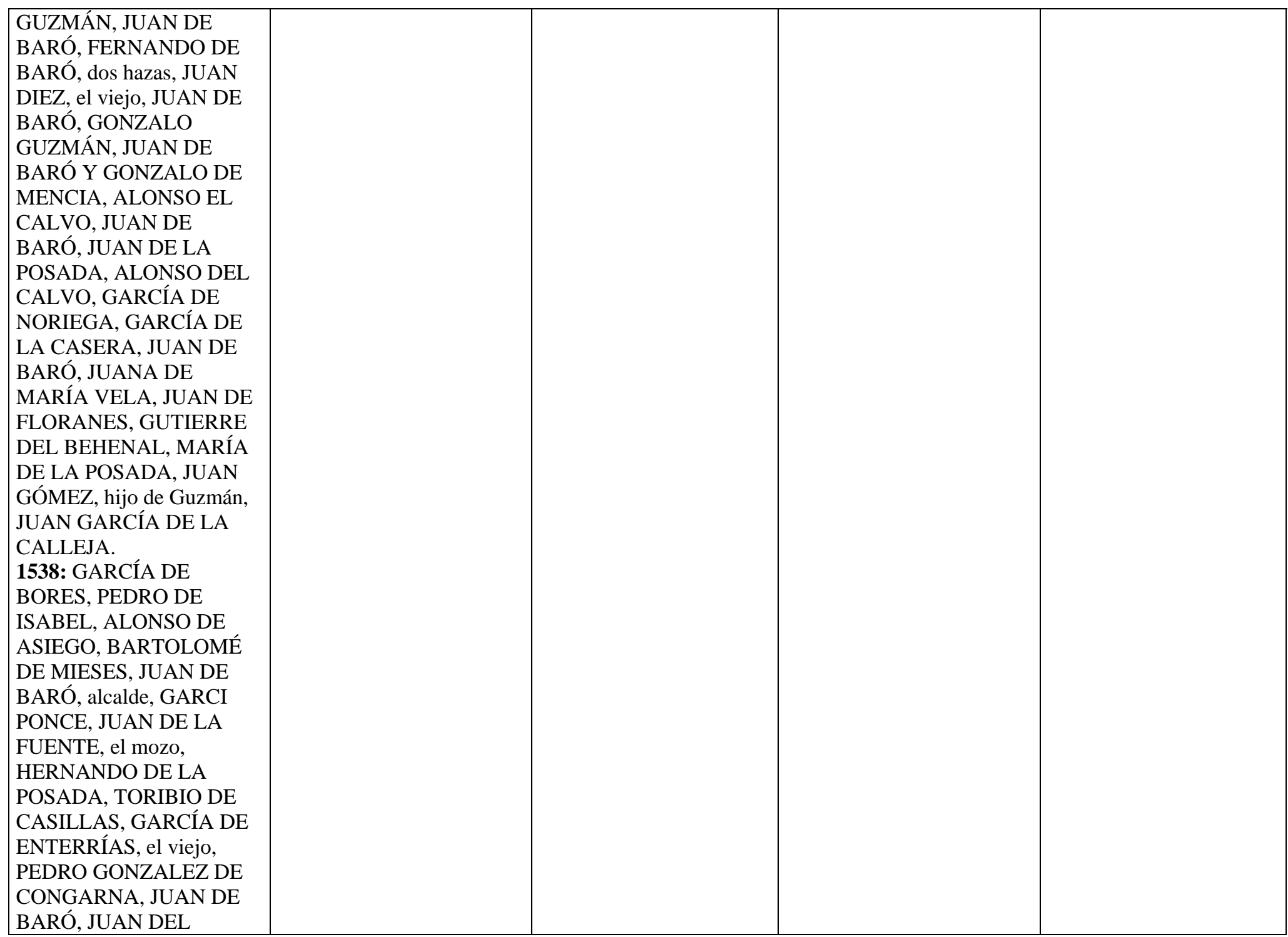




\begin{tabular}{|c|c|c|c|c|}
\hline $\begin{array}{l}\text { MERINO, GARCÍA DE } \\
\text { ENTERRÍAS, el viejo, } \\
\text { PEDRO CEREBANES, } \\
\text { JUAN RODRIGUEZ, hijo } \\
\text { de María de Fernando, } \\
\text { SANCHO DE BARÓ Y } \\
\text { ELVIRA DEL PONTÓN. }\end{array}$ & & & & \\
\hline $\begin{array}{l}\text { 1499: NO FIGURA } \\
\text { 1515: MARTIN DE JUAN } \\
\text { DE DIEGO } \\
\text { 1538: MARTIN DE } \\
\text { BARO, vecino de Turieno. }\end{array}$ & & $\begin{array}{l}1 \text { VIÑA en el bajo de San } \\
\text { Lázaro } \\
3 \text { CANTARAS DE VINO }\end{array}$ & & A TERCIO. \\
\hline $\begin{array}{l}\text { 1499: GONZALO DE } \\
\text { BARÓ, merino, ALONSO } \\
\text { DEL VALLE. } \\
\text { 1515: PEDRO DE LA } \\
\text { POSADA } \\
\text { 1538: NO FIGURA }\end{array}$ & $\begin{array}{l}1 \text { VIÑA en Viña Mayor } \\
\text { 4-5 CÁNTARAS DE VINO }\end{array}$ & 1 MIEDRO DE VINO & & ES DE TERCIO \\
\hline $\begin{array}{l}1499 \text { y 1515: SANCHO } \\
\text { ROMOROSO } \\
\text { 1538: ALONSO GOMEZ } \\
\text { DE MIESES }\end{array}$ & $\begin{array}{l}1 \text { VIÑA en Viña Mayor } \\
2 \text { MIEDROS DE VINO }\end{array}$ & & $\dot{¿} ?$ & A TERCIO. \\
\hline $\begin{array}{l}\text { 1499: ISABEL, mujer de } \\
\text { Juan de Noriega } \\
\text { 1515: PEDRO DE PIASCA } \\
\text { 1538: JUAN DE LA } \\
\text { ERMIDA }\end{array}$ & $\begin{array}{l}1 \text { VIÑA en Viña Mayor } \\
4 \text { CANTARAS DE VINO }\end{array}$ & & & POR TERCIO. \\
\hline & 1 VIÑA en Viña Mayor & & & POR TERCIO \\
\hline
\end{tabular}




\begin{tabular}{|c|c|c|c|}
\hline $\begin{array}{l}\text { 1499: ISABEL, mujer de } \\
\text { Juan de Noriega } \\
\text { 1515: GARCIAA DE } \\
\text { MOLLEDA } \\
\text { 1538: JUAN DE LA } \\
\text { ERMIDA }\end{array}$ & 4 CÁNTARAS DE VINO & $\begin{array}{l}4 \quad-5 \text { CÁNTARAS DE } \\
\text { VINO }\end{array}$ & \\
\hline $\begin{array}{l}1499 \text { y 1515: GARCÍA DE } \\
\text { NORIEGA } \\
\text { 1538: NO FIGURA }\end{array}$ & $\begin{array}{l}1 \text { VIÑA en Viña Mayor } \\
4 \text { CÁNTARAS DE VINO }\end{array}$ & 1 MIEDRO DE VINO & POR TERCIO \\
\hline $\begin{array}{l}1499 \text { y 1515: GONZALO } \\
\text { GUZMÁN } \\
\text { 1538: NO FIGURA }\end{array}$ & $\begin{array}{l}1 \text { VIÑA en Viña Mayor } \\
2-3 \text { CÁNTARAS DE } \\
\text { VINO }\end{array}$ & 3 CESTAS DE UVAS & POR TERCIO. \\
\hline $\begin{array}{l}\text { 1499: ISABEL, mujer de } \\
\text { Juan de Noriega } \\
\text { 1515: GARCÍA DE } \\
\text { NORIEGA } \\
\text { 1538: NO FIGURA }\end{array}$ & $\begin{array}{l}1 \text { VIÑA en Viña Mayor } \\
\dot{¿} ?\end{array}$ & 1 MIEDRO DE VINO & POR TERCIO. \\
\hline $\begin{array}{l}\text { 1499: JUAN DE } \\
\text { LAMASÓN Y TORIBIO, } \\
\text { hijo de Alonso Vallejo, } \\
\text { vecino de Potes } \\
\text { 1515: TORIBIO DE } \\
\text { PEDRO ORTEGA } \\
\text { 1538: NO FIGURA }\end{array}$ & $\begin{array}{l}1 \text { VIÑA en Viña Mayor } \\
2 \text { CÁNTARAS DE VINO }\end{array}$ & 1 CARGA DE UVAS & \\
\hline $\begin{array}{l}1499 \text { y 1515: DIEGO DE } \\
\text { BARÓ } \\
\text { 1538. NO FIGURA }\end{array}$ & $\begin{array}{l}1 \text { VIÑA en Tabejana } \\
1 \text { MIEDRO DE VINO }\end{array}$ & & A TERCIO. \\
\hline
\end{tabular}




\begin{tabular}{|c|c|c|c|}
\hline $\begin{array}{l}\text { 1499: CIERTAS } \\
\text { PERSONAS. } \\
\text { 1515: JUAN DIEZ DE } \\
\text { TURIENO, JUAN DE } \\
\text { BARÓ, MARÍA DE LA } \\
\text { POSADA, GONZALO, } \\
\text { hijo de Gonzalo de Baró, } \\
\text { JUAN, su heredero, DIEGO } \\
\text { DE BARÓ, PEDRO DE } \\
\text { PIASCA, GARCÍA DE } \\
\text { MOLLEDA Y OTROS. } \\
\text { 1538: NO FIGURA }\end{array}$ & $\begin{array}{l}1 \text { VIÑA en so Casa } \\
3 \text { - } 4 \text { MIEDROS DE VINO }\end{array}$ & $\begin{array}{l}5 \text { - } 6 \text { MIEDROS DE } \\
\text { VINO }\end{array}$ & ES DE TERCIO. \\
\hline $\begin{array}{l}\text { 1499: ALONSO DEL } \\
\text { VALLE, GÓMEZ DE } \\
\text { OTERO, ALONSO DEL } \\
\text { CALVO, MARÍA DE LA } \\
\text { POSADA, TERESA, la } \\
\text { casera, JUAN, hijo de } \\
\text { Martín Vallejo, JUAN } \\
\text { DÍEZ DE TURIENO, } \\
\text { HEREDEROS DE PEDRO } \\
\text { VALLEJO. } \\
\text { 1515: ALONSO DE } \\
\text { OCEÑO, MARÍA DE LA } \\
\text { POSADA, ALONSO EL } \\
\text { CALVO, JUAN GÓMEZ, } \\
\text { ALONSO DEL VALLE, } \\
\text { GARCÍA DE TERESA Y } \\
\text { JUAN DÍEZ DE TURIENO } \\
\text { 1538: TORIBIO, hijo de } \\
\text { Sancho de Casillas, } \\
\text { ALONSO DE OCEÑO, } \\
\text { GARCI PONCE, ISABEL } \\
\text { DE MIESES, FERNANDO }\end{array}$ & $\begin{array}{l}1 \text { VIÑA en Llandecilla } \\
20 \text { MIEDROS DE VINO }\end{array}$ & $\begin{array}{l}20-25 \text { MIEDROS DE } \\
\text { VINO }\end{array}$ & $\begin{array}{lll}\text { LA } & \text { LLEVAN } & \text { POR } \\
\text { TERCIO. } & \end{array}$ \\
\hline
\end{tabular}




\begin{tabular}{|c|c|c|c|}
\hline $\begin{array}{l}\text { DE LA POSADA Y } \\
\text { OTROS. }\end{array}$ & & & \\
\hline $\begin{array}{l}\text { 1499 y 1515: FERRÁN } \\
\text { LÓPEZ DE LAMADRID } \\
\text { 1538: ¿? }\end{array}$ & $\begin{array}{l}1 \text { VIÑA en Llandeçilla } \\
4 \text { CÁNTARAS DE VINO }\end{array}$ & 1 MIEDRO DE VINO & POR TERCIO. \\
\hline $\begin{array}{l}\text { 1499: CIERTAS } \\
\text { PERSONAS } \\
\text { 1515: FERRÁN BARÓ, } \\
\text { TORIBIO DE VALVERDE } \\
\text { DE POTES, JUAN DE } \\
\text { TERESA, ALONSO, su } \\
\text { heredero, JUAN GÓMEZ, } \\
\text { hijo de Gonzalo Guzmán, } \\
\text { ALONSO GARCÍA DE } \\
\text { CASTILLO, JUAN DE LA } \\
\text { CASA, JUAN GOMEZ DE } \\
\text { VEDOYA, ALONSO } \\
\text { TORRE, ALONSO DE } \\
\text { NORIEGA, TORIBIO DEL } \\
\text { BIERZO, GARCÍA } \\
\text { HERRERO, EL TEJEDOR, } \\
\text { PEDRO, el ROjo, } \\
\text { ALONSO CALDERÓN, } \\
\text { JUAN DE LOS COS, } \\
\text { ALONSO GARCÍA DE LA } \\
\text { CANAL, FERRÁN } \\
\text { GUTIERREZ DE } \\
\text { LINARES, JUAN DE } \\
\text { HIJÓN, GUTIERRE DEL } \\
\text { BEHENAL, JUAN DE LA } \\
\text { POSADA Y OTROS Y } \\
\text { JUAN DE LA CALLEJA. } \\
\text { 1538: JUAN DE }\end{array}$ & $\begin{array}{l}1 \text { VIÑA en la Serna Pumar } \\
30 \text { MIEDROS DE VINO }\end{array}$ & 20 MIEDROS DE VINO & POR TERCIO. \\
\hline
\end{tabular}




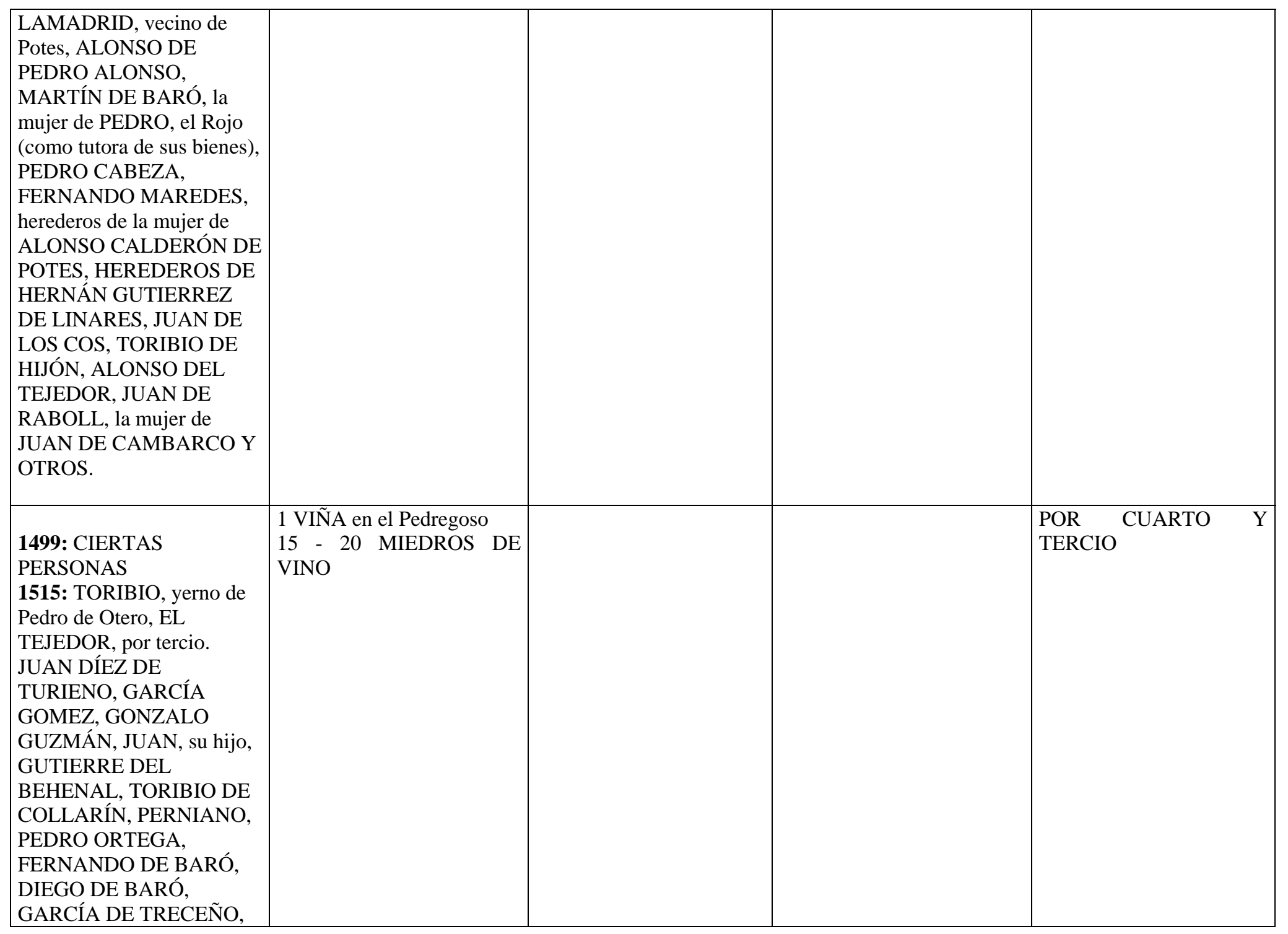




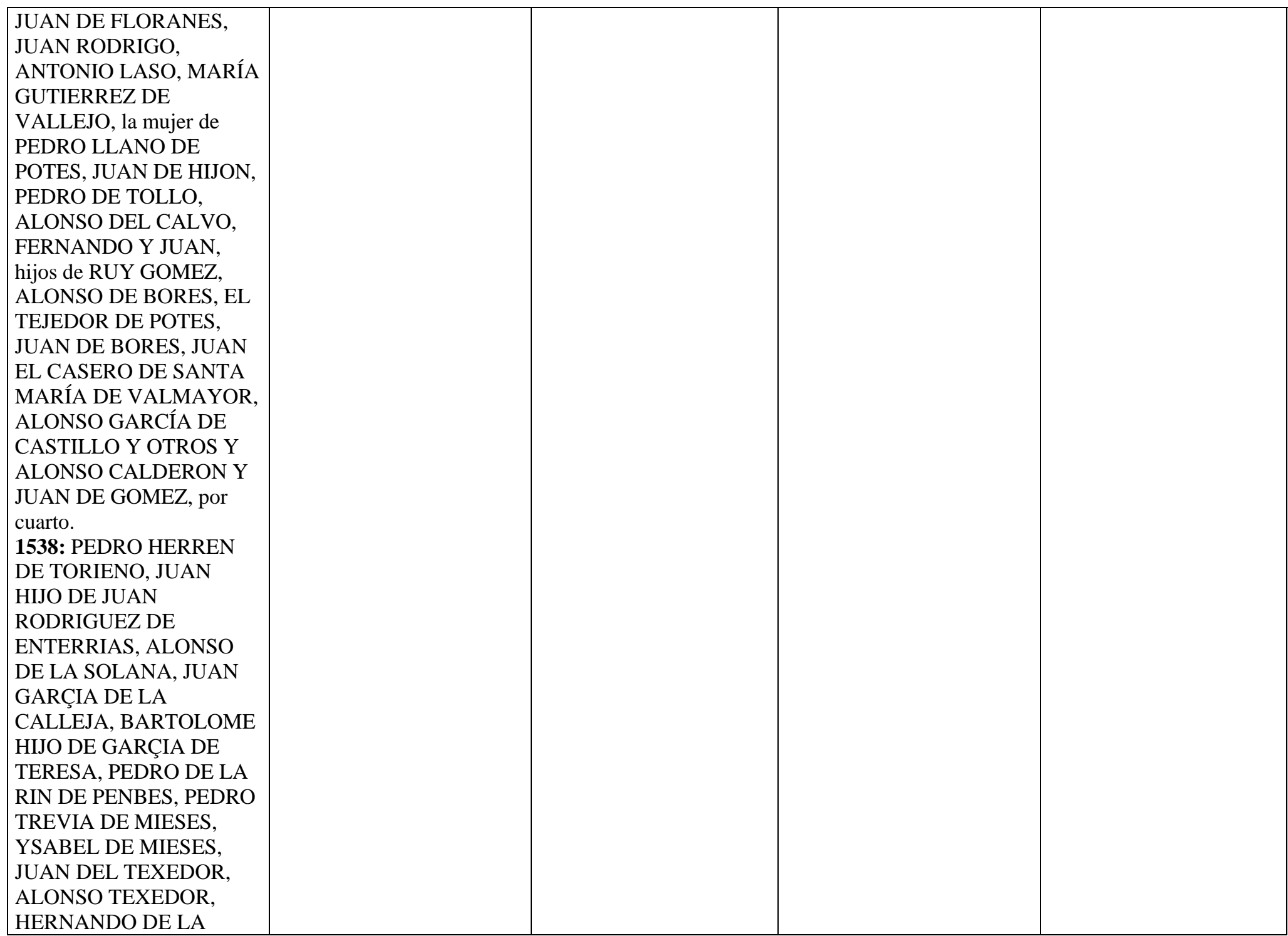




\begin{tabular}{|l|l|l|l|}
\hline POSADA, ANTONIO & & & \\
LASO DE MOGROVEJO, & & & \\
DIEGO DE VARO, LOS & & & \\
HEREDEROS DEL & & & \\
CALVO, FERNANDO DE & & & \\
RASAS, ELVIRA & & & \\
FERNANDEZ DE & & & \\
FLORANES Y OTROS. & & & \\
\hline
\end{tabular}

\section{HEREDADES A TERCIO EN POTES}

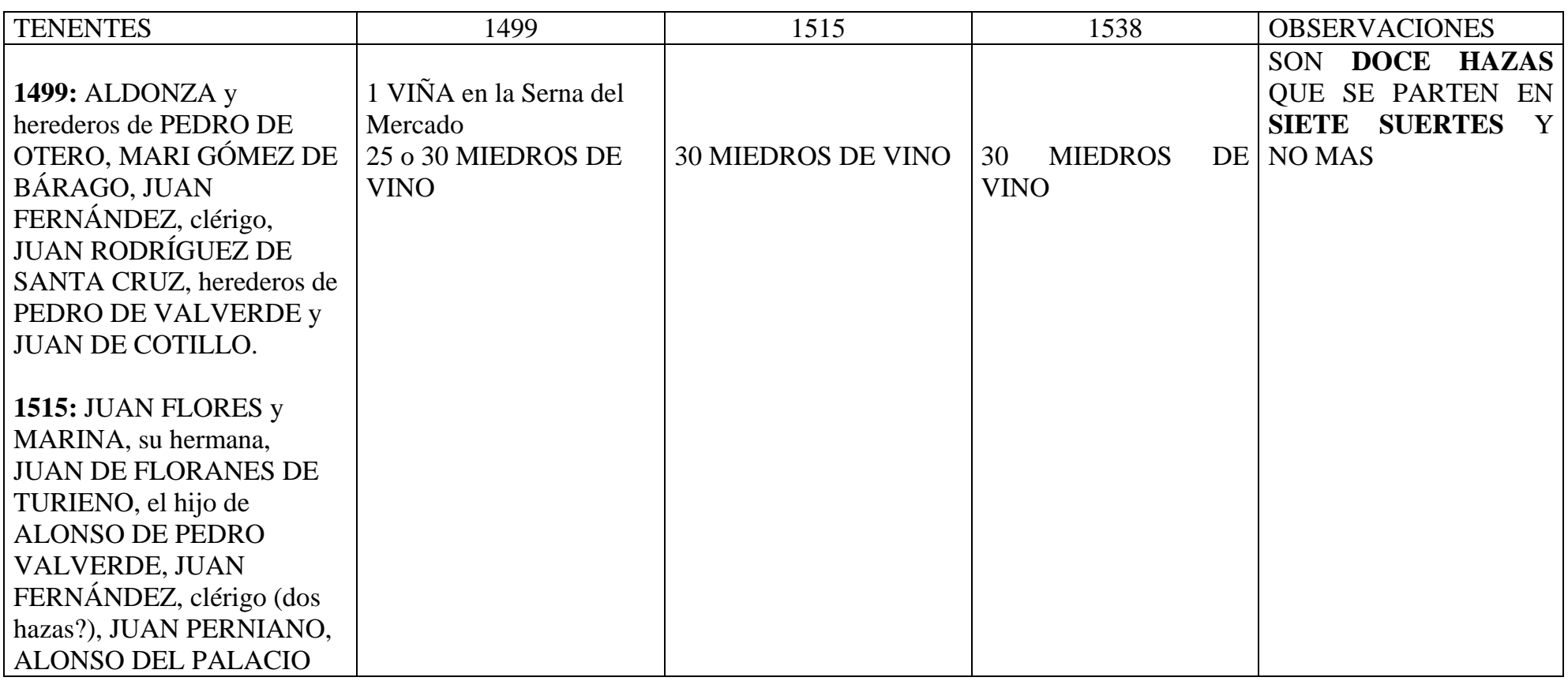




\begin{tabular}{|c|c|c|c|c|}
\hline $\begin{array}{l}\text { DE BARAGO, hijos de } \\
\text { PEDRO MUS, DIEGO DE } \\
\text { ARCE, JUAN DE } \\
\text { FERRANDO DE BARÓ, } \\
\text { ALONSO FERNÁNDEZ } \\
\text { 1538: Llevanla MUCHAS } \\
\text { PERSONAS DE LA VILLA }\end{array}$ & & & & \\
\hline $\begin{array}{l}\text { 1499: RODRIGO } \\
\text { BIENHECHO } \\
\text { 1515 y 1538: TORIBIO } \\
\text { PEREZ } \\
\end{array}$ & $\begin{array}{l}1 \text { HAZA DE VIÑA entre } \\
\text { las huertas. } \\
1 \text { MIEDRO DE VINO }\end{array}$ & & & 1499: por CUARTO \\
\hline $\begin{array}{l}1499 \text { y 1515: ALONSO } \\
\text { GARCÍA DE TOLLO, } \\
\text { merino y FERRAN LÓPEZ } \\
\text { DE LAMADRID } \\
\text { 1538: JUAN LÓPEZ DE } \\
\text { LAMADRID }\end{array}$ & $\begin{array}{l}1 \text { VIÑA en el Bo } \\
\text { 3-4 MIEDROS DE VINO }\end{array}$ & 4 MIEDROS DE VINO & 2 MIEDROS DE VINO & $\begin{array}{l}\text { Ha de estar en un } \\
\text { heredero y no más. }\end{array}$ \\
\hline $\begin{array}{l}\text { 1499: DIEGO DE } \\
\text { MOLLEDA } \\
\text { 1515: DIEGO GONZÁLEZ } \\
\text { DE MOLLEDA } \\
\text { 1538: SANCHO } \\
\text { FERNÁNDEZ DE LA VEGA }\end{array}$ & $\begin{array}{l}1 \text { VIÑA en el Barrio } \\
\dot{¿} ?\end{array}$ & 2 CÁNTARAS DE VINO & $\dot{¿} ?$ & Es de CUARTO. \\
\hline $\begin{array}{l}\text { 1499: GÓMEZ GAYÁN y su } \\
\text { madre SANCHA BIELVA } \\
\text { 1515: JUAN DE LAYO y }\end{array}$ & $\begin{array}{l}1 \text { VIÑA entre ambas } \\
\text { riegas } \\
3 \text { MIEDROS DE VINO }\end{array}$ & 5 MIEDROS DE VINO & 5 MIEDROS DE VINO & \\
\hline
\end{tabular}




\begin{tabular}{|c|c|c|c|c|}
\hline $\begin{array}{l}\text { PEDRO DE LAYO } \\
\text { 1538: JUAN DE LAYO }\end{array}$ & & & & \\
\hline $\begin{array}{l}\text { 1499: GÓMEZ GAYÁN } \\
\text { 1515: JUAN DE LAYO E } \\
\text { PEDRO DE LAYO } \\
\text { 1538: JUAN DE LAYO, } \\
\text { vecino de Potes }\end{array}$ & $\begin{array}{l}1 \text { VIÑA en Palacio } \\
1 \text { MIEDRO DE VINO }\end{array}$ & & & $\begin{array}{l}\text { Dicha viña la dio en } \\
\text { enmienda Ferran López } \\
\text { de Lamadrid por otra que } \\
\text { estaba en la Cabaña. }\end{array}$ \\
\hline $\begin{array}{l}\text { 1499: JUAN GARCÍA DE } \\
\text { BARNIEDO, JUAN TEJEDOR, } \\
\text { JUAN DE ENCIMA EL } \\
\text { CORRAL } \\
\text { 1515: ALONSO DE PEDRO } \\
\text { BONO, PEDRO DE LLES y } \\
\text { JUAN TEJEDOR } \\
\text { 1538: JUANA, mujer de } \\
\text { PEDRO LLES }\end{array}$ & $\begin{array}{l}1 \text { VIÑA en la Cobilla del } \\
\text { Gato } \\
3 \text { MIEDROS DE VINO }\end{array}$ & & & \\
\hline 1515: MARI TURA & NO FIGURA & $\begin{array}{l}1 \text { VIÑA entre las huertas } \\
2 \text { CARGAS DE UVAS }\end{array}$ & NO FIGURA & \\
\hline $\begin{array}{l}\text { 1499: JUAN DE LA } \\
\text { CASANUEVA } \\
\text { 1515: JUAN, hijo de } \\
\text { NORIEGA }\end{array}$ & $\begin{array}{l}1 \text { TIERRA en la } \\
\text { Escontrilla, término de } \\
\text { Valmeo } \\
1 \text { CUARTO DE TRIGO }\end{array}$ & & & 1499: Por CUARTO \\
\hline
\end{tabular}




\begin{tabular}{|c|c|c|c|c|}
\hline $\begin{array}{l}\text { 1499: TORIBIO HERRERO } \\
\text { 1515: ALONSO } \\
\text { CALDERÓN } \\
\text { 1538: HERNANDO DE } \\
\text { MARTÍ y TERESA DE LA } \\
\text { COMINERA }\end{array}$ & $\begin{array}{l}1 \text { VIÑA en Helvanes } \\
2 \text { MIEDROS DE VINO }\end{array}$ & $\begin{array}{l}1 \text { VIÑA en el Açeval } \\
2 \text { MIEDROS DE VINO }\end{array}$ & $\begin{array}{l}1 \text { TIERRA en Açeval } \\
1 \text { CUARTO DE PAN }\end{array}$ & $\begin{array}{lr}\text { La lleva por } & \text { CUARTO } \\
\text { (1499 y 1515). } & \\
\text { (1538): PAGA } & \mathbf{1} \\
\text { SESTERO } & \text { DE } \\
\text { TRIGO } & \text { DE } \\
\text { INFURCIÓN. } & \end{array}$ \\
\hline $\begin{array}{l}\text { 1499 y 1515: JUAN DE } \\
\text { LAMASON } \\
\text { 1538: PEDRO SÁNCHEZ } \\
\text { CALDERÓN }\end{array}$ & $\begin{array}{l}1 \text { TIERRA en Llan de } \\
\text { Vurlandero } \\
6 \text { EMINAS DE TRIGO }\end{array}$ & & 7 CUARTOS TRIGO & La lleva por CUARTO \\
\hline
\end{tabular}




\begin{tabular}{|c|c|c|c|c|}
\hline $\begin{array}{l}\text { 1499: PEDRO DE HIJÓN, } \\
\text { TORIBIO CHINCHILLA, } \\
\text { herederos de JUAN DE } \\
\text { OTERO, GARCÍA } \\
\text { HERRERO, JUAN DE } \\
\text { COTILLO, JUAN DE } \\
\text { FERNANDO DE RASES y } \\
\text { OTROS. } \\
\text { 1515: JUAN DE HIJÓN, } \\
\text { MARIA BIELVA, GARCÍA } \\
\text { HERRERO, JUAN DE } \\
\text { OTERO, JUAN PERNIANO, } \\
\text { FERRANDO DE RASES } \\
\text { 1538: TORIBIO DE JUAN } \\
\text { DE OTERO, vecino de Potes, } \\
\text { FERNANDO DE RASES, } \\
\text { JUAN GONZÁLEZ } \\
\text { PERNIANO y OTROS. }\end{array}$ & $\begin{array}{l}1 \text { VIÑA en el Calero } \\
15 \text { MIEDROS DE VINO }\end{array}$ & & & $\begin{array}{l}\text { Han de llevarla } \\
\text { CUATRO } \\
\text { HEREDEROS y no } \\
\text { más. } \\
\text { ES DE TERCIO } \\
\text { 1499: La dicha viña es } \\
\text { SERNA REDONDA. }\end{array}$ \\
\hline $\begin{array}{l}\text { 1499: GONZALO } \\
\text { RODRÍGUEZ y ALONSO } \\
\text { GARCÍA, su heredero. } \\
\text { 1515: ALONSO GARCÍA } \\
\text { DE LA CANAL } \\
\text { 1538: PEDRO SÁNCHEZ } \\
\text { DE CALDAS }\end{array}$ & $\begin{array}{l}1 \text { TIERRA en Helvanes } \\
1 \text { CUARTO DE TRIGO }\end{array}$ & 11⁄2 CUARTO de TRIGO & & Es de TERCIO \\
\hline $\begin{array}{l}\text { 1499: JUAN GARCÍA DE } \\
\text { BARNIEDO, JUAN } \\
\text { TEJEDOR, JUAN DE }\end{array}$ & $\begin{array}{l}1 \text { TIERRA (que solía ser } \\
\text { viña) encima del corral } \\
2 \text { FANEGAS DE TRIGO }\end{array}$ & 3 FANEGAS DE PAN & 3 FANEGAS DE PAN & $\begin{array}{l}\text { Es de TERCIO } \\
\text { 1499: Está partida en } \\
\text { MUCHAS HAZAS. }\end{array}$ \\
\hline
\end{tabular}




\begin{tabular}{|c|c|c|c|}
\hline $\begin{array}{l}\text { JUSTO, RODRIGO } \\
\text { BIENHECHO, TORIBIO DE } \\
\text { MARTINO } \\
\text { 1515: JUAN DE OTERO } \\
\text { 1538: TORIBIO ORTEGA y } \\
\text { ALONSO GÓMEZ, su } \\
\text { hermano. }\end{array}$ & & & \\
\hline $\begin{array}{l}\text { 1499: TORIBIO DE } \\
\text { MARTÍN y JUAN, hijo de } \\
\text { RODRIGO BIENHECHO } \\
\text { 1515: JUAN DE OTERO } \\
\text { 1538: NO FIGURA }\end{array}$ & $\begin{array}{l}1 \text { TIERRA a la puente de } \\
\text { Deva } \\
1 \text { SESTERO DE TRIGO }\end{array}$ & 6 HEMINAS DE PAN & $\begin{array}{l}\text { 1499: es de TERCIO } \\
\text { (1515) En el margen: } \\
\text { TROCOSE por un prado } \\
\text { en Argüébanes }\end{array}$ \\
\hline $\begin{array}{l}\text { 1499: LLEVAN CIERTAS } \\
\text { PERSONAS. } \\
\text { 1515: FERRAN LÓPEZ, } \\
\text { TORIBIO DE BARAGO, } \\
\text { JUAN DE OTERO, } \\
\text { PEREJON, PEDRO } \\
\text { REVUELTA, TORIBIO DE } \\
\text { VALVERDE, JUAN DE } \\
\text { HIJON, JUAN DE } \\
\text { AGUEROS, GARCIA } \\
\text { HERRERO, la hija de } \\
\text { ALONSO TORRE, } \\
\text { PERNIANO, MARIA } \\
\text { BIELVA, herederOS de } \\
\text { PEDRO MUS, PEDRO DE } \\
\text { LAYO, JUAN } \\
\text { FERNÁNDEZ, FERRAN } \\
\text { GUTIERREZ DE LINARES, }\end{array}$ & $\begin{array}{l}1 \text { VIÑA en la Pereda } \\
20-25 \text { MIEDROS DE } \\
\text { VINO }\end{array}$ & & $\begin{array}{l}\text {-ESTA HECHA } \\
\text { SUERTES } \\
\text {-ES DE TERCIO }\end{array}$ \\
\hline
\end{tabular}




\begin{tabular}{|c|c|c|c|c|}
\hline $\begin{array}{l}\text { ALONSO DE BORES, } \\
\text { TORIBIO DE LAMADRID, } \\
\text { el “ROJO”, JUAN DE } \\
\text { CAMBARCO, TORIBIO } \\
\text { CORTINA, TORIBIO DE } \\
\text { LA CUESTA DE } \\
\text { VALVERDE, JUAN DE } \\
\text { LOS COS y OTROS. } \\
\text { 1538: JUAN DE } \\
\text { LAMADRID, PERNIANO, } \\
\text { DIEGO FERNÁNDEZ DE } \\
\text { MARITA [sic] y OTRAS } \\
\text { PERSONAS }\end{array}$ & & & & \\
\hline $\begin{array}{l}\text { 1499 y 1515: FERRANDO } \\
\text { DE LA CUESTA } \\
\text { 1538: PEDRO HERRERO, } \\
\text { vecino de Potes }\end{array}$ & $\begin{array}{l}1 \text { VIÑA en el Hoyal } \\
1 \text { MIEDRO DE VINO }\end{array}$ & 2 CARGAS DE UVAS & 1 MIEDRO DE VINO & Es de TERCIO \\
\hline $\begin{array}{l}\text { 1499: La mujer de ALONSO } \\
\text { DE COTILLO y JUAN DE } \\
\text { FERRANDO DE RASES } \\
\text { 1515: FERRANDO RASES y } \\
\text { PERNIANO } \\
\text { 1538: HERNANDO DE } \\
\text { RASES }\end{array}$ & $\begin{array}{l}1 \text { VIÑA en Haravedes } \\
4 \text { CANTARAS DE VINO }\end{array}$ & & 2 CARGAS DE UVAS & La llevan por CUARTO \\
\hline
\end{tabular}




\begin{tabular}{|c|c|c|c|c|}
\hline $\begin{array}{l}\text { 1499: RODRIGO } \\
\text { BIENHECHO, RODRIGO } \\
\text { DE JUAN, GONZALO DEL } \\
\text { ARCIPRESTE, JUAN DE } \\
\text { PANES, PEDRO PÉREZ, } \\
\text { TORIBIO HERRERO, } \\
\text { GÓMEZ GAYÁN, } \\
\text { ALDONZA y FERRAN } \\
\text { LÓPEZ (que lleva la suerte de } \\
\text { Mari Barreda) } \\
\text { 1515: RUY GOMEZ, } \\
\text { GONZALO FERNÁNDEZ } \\
\text { DE ZANARA (dos hazas?), } \\
\text { PEDRO JUSTES, PEDRO } \\
\text { DE LAYO, JUAN DE } \\
\text { LAYO, FERRAN LÓPEZ, } \\
\text { ALDONZA, JUAN FLORES, } \\
\text { CALDERÓN y ALONSO DE } \\
\text { PANES DE ARGÜÉBANES } \\
\text { 1538: JUAN DE LAYO, } \\
\text { SANCHO DE NORIEGA, } \\
\text { HERNANDO DE MARTÍN, } \\
\text { ALONSO FLORES, } \\
\text { ALONSO TEJEDOR, JUAN } \\
\text { DE ARCE, JUAN } \\
\text { ROBERTO, JUAN DE } \\
\text { LAMADRID, JUAN } \\
\text { GARCÍA DEL HERRERO y } \\
\text { BARTOLOMÉ GARCÍA. }\end{array}$ & $\begin{array}{l}1 \text { VIÑA en el Hoyo } \\
6 \text { o } 7 \text { MIEDROS DE } \\
\text { VINO }\end{array}$ & 7 MIEDROS DE VINO & 9 MIEDROS DE VINO & \\
\hline $\begin{array}{l}\text { 1499: JUAN DE GOLLO y } \\
\text { JUAN, hijo de RODRIGO } \\
\text { BIENHECHO } \\
\text { 1515: JUAN DE GOLLO }\end{array}$ & $\begin{array}{l}1 \text { PANEDO en el Hoyo } \\
1 \text { HEMINA DE PAN }\end{array}$ & & ESTÁ DESPOBLADA & Lleva por CUARTO. \\
\hline 1499 y 1515: FERRAN & & & & 1499 y 1515: Lleva por \\
\hline
\end{tabular}




\begin{tabular}{|c|c|c|c|c|}
\hline $\begin{array}{l}\text { GUTIERREZ DE LINARES } \\
\text { 1538: ELVIRA, mujer que } \\
\text { fue del “ROJO”" }\end{array}$ & $\begin{array}{l}1 \text { TIERRA en el Hoyo } \\
1 \text { CUARTO DE TRIGO }\end{array}$ & 6 EMINAS DE TRIGO & 1 FANEGA DE PAN & $\begin{array}{l}\text { CUARTO. } \\
\text { 1538: Es de TERCIO. }\end{array}$ \\
\hline $\begin{array}{l}\text { 1499: ALONSO DE } \\
\text { FLORANES DE POTES } \\
\text { 1515: MARINA, mujer que } \\
\text { fue de ALONSO DE } \\
\text { FLORANES } \\
\text { 1538: NO FIGURA }\end{array}$ & $\begin{array}{l}1 \text { VIÑA en la Moneca } \\
2 \text { CANTARAS DE VINO }\end{array}$ & 2 CARGAS DE UVAS & $\begin{array}{l}1 \quad \text { PANEDO en la } \\
\text { Moneca } \\
1 \text { SESTERO DE } \\
\text { TRIGO }\end{array}$ & $\begin{array}{l}\text { 1499 Y 1515: Por } \\
\text { CUARTO }\end{array}$ \\
\hline $\begin{array}{l}1499 \text { y 1515: JUAN } \\
\text { GARCÍA DE BORES } \\
\text { 1538: GARCÍA DE COSIO }\end{array}$ & $\begin{array}{l}1 \text { VIÑA en Santolaja } \\
7 \text { u } 8 \text { MIEDROS DE } \\
\text { VINO }\end{array}$ & & & Es de TERCIO \\
\hline $\begin{array}{l}\text { 1499 y 1515: GOMEZ DE } \\
\text { SALCEDA y JUAN DE LA } \\
\text { FRANCA } \\
\text { 1538: JUAN DE MARTÍN y } \\
\text { FRANCISCO, el viejo }\end{array}$ & $\begin{array}{l}1 \text { VIÑA en término de } \\
\text { Santolaja } \\
5 \text { MIEDROS DE VINO }\end{array}$ & & $\begin{array}{l}7 \text { u } 8 \text { MIEDROS DE } \\
\text { VINO }\end{array}$ & $\begin{array}{l}\text { Es de TERCIO. } \\
\text { 1499: Tiene que quedar } \\
\text { en } 1 \text { HEREDERO Y NO } \\
\text { MAS }\end{array}$ \\
\hline $\begin{array}{l}\text { 1499: DIEGO GONZÁLEZ } \\
\text { DE LAMADRID } \\
\text { 1515: Herederos de DIEGO } \\
\text { GONZALEZ DE } \\
\text { LAMADRID } \\
\text { 1538: SANCHO } \\
\text { GONZÁLEZ DE NORIEGA }\end{array}$ & $\begin{array}{l}1 \text { VIÑA en Campañana } \\
2 \text { a } 3 \text { MIEDROS DE } \\
\text { VINO }\end{array}$ & & $\begin{array}{l}1 \text { VIÑA y TIERRA en } \\
\text { Campañana } \\
1 \text { EMINA DE TRIGO } \\
3 \text { o } 4 \text { CARGAS DE } \\
\text { UVAS }\end{array}$ & Es de TERCIO \\
\hline $\begin{array}{l}\text { 1499: MARINA DE } \\
\text { MALAHEMBRA } \\
\text { 1515: JUAN DE } \\
\text { CAMBARCO } \\
\text { 1538: Herederos de JUAN } \\
\text { DE CAMBARCO. } \\
\end{array}$ & $\begin{array}{l}1 \text { VIÑA en Palombierzo } \\
1 \text { CESTO DE UVAS }\end{array}$ & 2 CARGAS DE UVAS & 2 CARGAS DE UVAS & La lleva por CUARTO \\
\hline
\end{tabular}


CONCEJO DE LEBEÑA. VIÑAS DE TERCIO

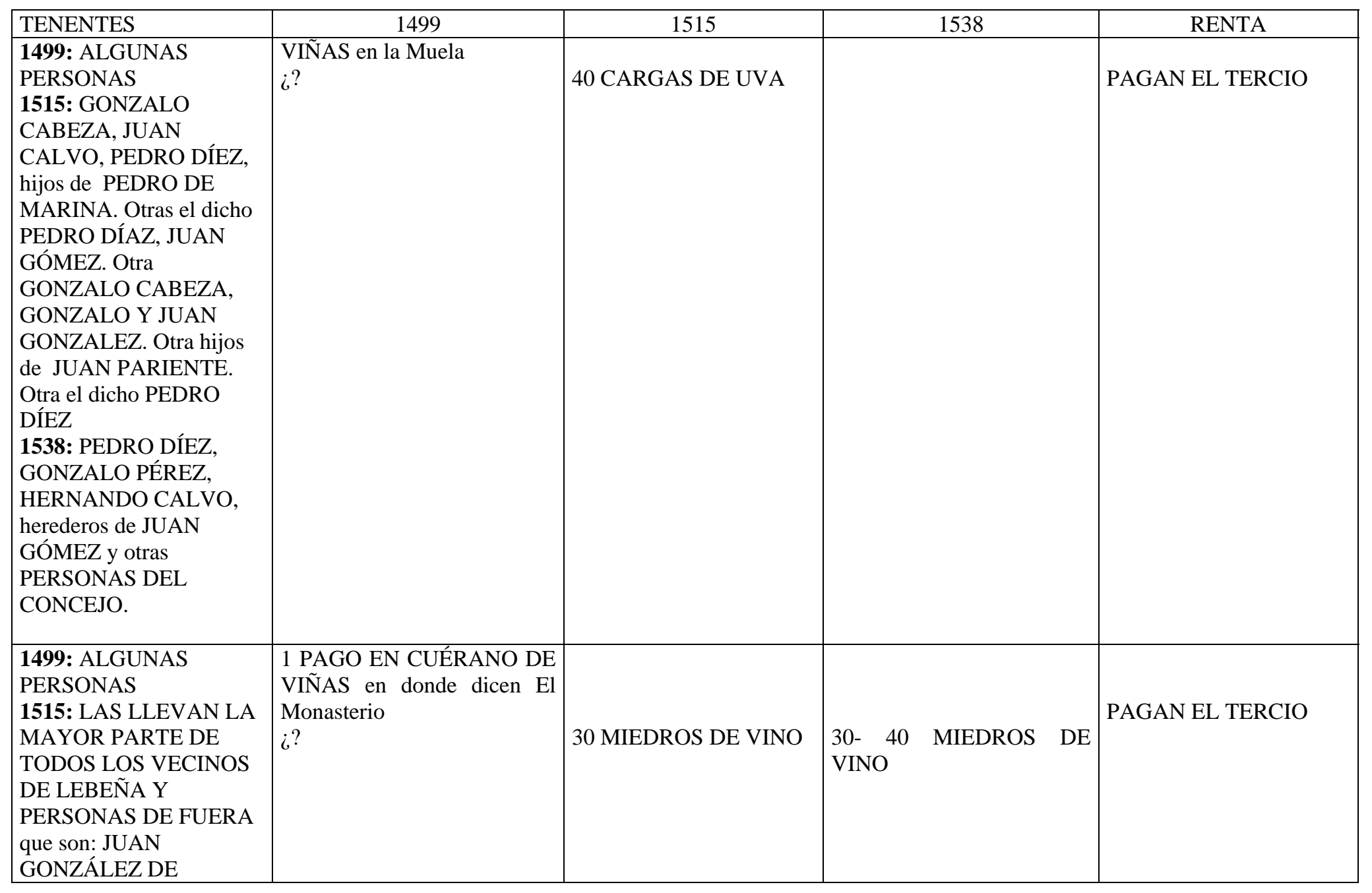




\begin{tabular}{|c|c|c|c|c|}
\hline $\begin{array}{l}\text { PENDES, GUTIERRE } \\
\text { GÓMEZ DE PENDES, la } \\
\text { mujer de JUAN } \\
\text { HERRERO, INÉS DE } \\
\text { SALARZÓN, MARÍA } \\
\text { GONZÁLEZ DE JUAN } \\
\text { DE LLES, MARÍA } \\
\text { PIMERAS Y ALONSO, } \\
\text { hijo de GARCÍA DE } \\
\text { JUAN GARCÍA, vecino } \\
\text { de Cabañes } \\
\text { 1538: RODRIGO } \\
\text { GARCÍA, JUAN DEL } \\
\text { ARENAL, GOMEZ } \\
\text { GARCÍA Y OTRAS } \\
\text { PERSONAS DEL } \\
\text { CONCEJO. }\end{array}$ & & & & \\
\hline $\begin{array}{l}\text { 1499: ALGUNAS } \\
\text { PERSONAS } \\
\text { 1515: ALONSO DEL } \\
\text { VALLE, PEDRO DEL } \\
\text { VALLE, JUAN DE } \\
\text { COSIO, GUTIERRE DEL } \\
\text { VALLE, JUAN, hijo de } \\
\text { GARCÍA CALVO Y } \\
\text { OTROS } \\
\text { 1538: NO FIGURA }\end{array}$ & $\begin{array}{l}1 \text { PAGO EN CUERANO DE } \\
\text { VIÑAS en la Huerta Redonda } \\
\dot{¿} ?\end{array}$ & 10 MIEDROS DE VINO & & $\begin{array}{l}\text { ESTAS TRES VIÑAS } \\
\text { SON UNA PORQUE } \\
\text { ESTAN JUNTAS. } \\
\text { PAGAN EL TERCIO }\end{array}$ \\
\hline $\begin{array}{l}\text { 1499: ALGUNAS } \\
\text { PERSONAS } \\
\text { 1515: GONZALO } \\
\text { CABEZA, GUTIERRE } \\
\text { GÓMEZ DE PENDES, } \\
\text { PEDRO DEL VALLE, } \\
\text { JUAN CALVO, JUAN } \\
\text { DE COSIO, JUAN }\end{array}$ & $\begin{array}{l}1 \text { PAGO Y CUERANO DE } \\
\text { VIÑAS sobre la iglesia de } \\
\text { Santa María } \\
\dot{¿} \text { ? }\end{array}$ & 25 MIEDROS DE VINO & 30 MIEDROS DE VINO & PAGAN EL TERCIO \\
\hline
\end{tabular}




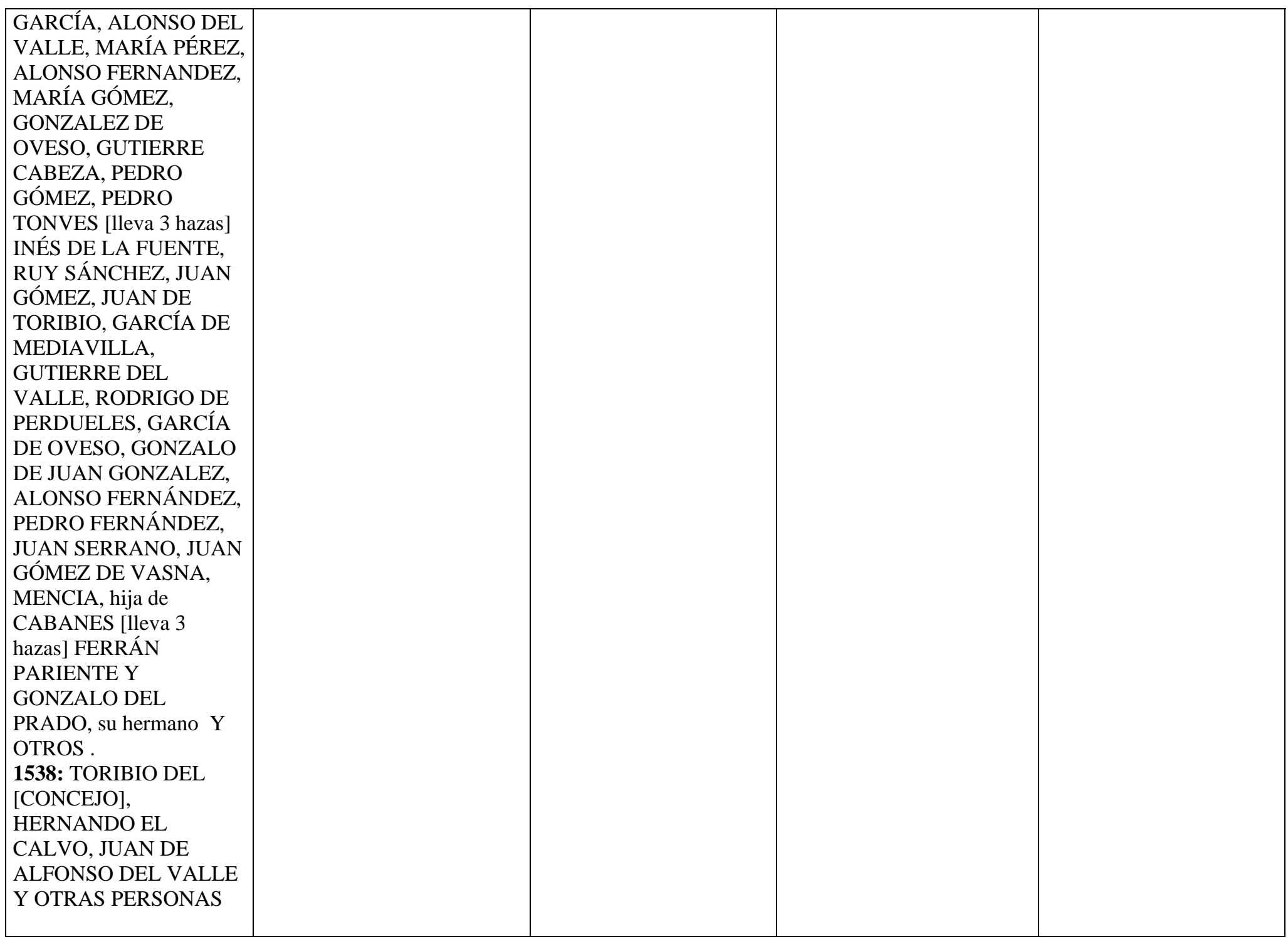




\begin{tabular}{|c|c|c|c|c|}
\hline $\begin{array}{l}\text { 1499: ALGUNAS } \\
\text { PERSONAS } \\
\text { 1515: PEDRO GÓMEZ, } \\
\text { FERRÁN PARIENTE, } \\
\text { FERNANDO DE } \\
\text { LEVEÑA, JUAN DE } \\
\text { CAMPOS, MARÍA DE } \\
\text { GUTIERRE GÓMEZ, } \\
\text { INÉS DE LA FUENTE Y } \\
\text { OTROS. } \\
\text { 1538: HERNÁN } \\
\text { PARIENTE, TORIBIO } \\
\text { GARCÍA, JUAN DE } \\
\text { PEDRO DÍEZ y OTRAS } \\
\text { PERSONAS }\end{array}$ & $\begin{array}{l}1 \text { VIÑA en Granada } \\
\dot{¿} \text { ? }\end{array}$ & 5 MIEDROS DE VINO & & PAGAN EL TERCIO \\
\hline $\begin{array}{l}\text { 1499 y 1515: Hijos de } \\
\text { GONZALO CALVO, } \\
\text { JUAN GÓMEZ y } \\
\text { OTROS. } \\
\text { 1538: HERNAN } \\
\text { CALVO, CELES y } \\
\text { OTRAS PERSONAS }\end{array}$ & $\begin{array}{l}1 \text { VIÑA en la Población } \\
\dot{¿} \text { ? }\end{array}$ & Ilegible & 1 MIEDRO DE VINO & PAGAN EL TERCIO \\
\hline $\begin{array}{l}\text { 1499 y 1515: TORIBIO } \\
\text { DE MEDIAVILLA } \\
\text { 1538: ALONSO } \\
\text { FERNÁNDEZ }\end{array}$ & $\begin{array}{l}1 \text { VIÑA en tras Quindio } \\
\dot{¿} \text { ? }\end{array}$ & 4 CANTARAS DE VINO & 3 CARGAS DE UVAS & PAGA EL TERCIO \\
\hline
\end{tabular}




\section{HEREDADES EN EL CONCEJO DE COLIO}

\begin{tabular}{|c|c|c|c|}
\hline ARRENDATARIO & 1499 & 1515 & 1538 \\
\hline \multirow{8}{*}{$\begin{array}{l}\text { LAS LLEVAN LOS DEL } \\
\text { CONCEJO DE COLIO }\end{array}$} & $\begin{array}{l}1 \text { TIERRA que se dice la Serna de } \\
\text { sobre Santyago } \\
2 \text { 1/2 FANEGAS DE TRIGO }\end{array}$ & & \\
\hline & $\begin{array}{l}1 \text { TIERRA en Corteçylla } \\
3 \text { HEMINAS DE TRIGO }\end{array}$ & & \\
\hline & $\begin{array}{l}1 \text { TIERRA entranbas carreras } \\
1 \text { HEMINA DE PAN }\end{array}$ & 1 HEMINA DE TRIGO & \\
\hline & $\begin{array}{l}1 \text { TIERRA a la fuente del Gallegar } \\
1 \text { SESTERO DE TRIGO }\end{array}$ & & 1 SESTERO DE PAN \\
\hline & $\begin{array}{l}1 \text { TIERRA en Cerceda } \\
1 \text { CUARTO DE PAN }\end{array}$ & & \\
\hline & $\begin{array}{l}1 \text { PRADO junto con la tierra de } \\
\text { Cerceda } \\
10 \text { CARGAS DE YERBA los } 1 / 2 \\
\text { años }\end{array}$ & & 5 CARGAS DE YERBA \\
\hline & $\begin{array}{l}1 \text { TIERRA en el llano de Piedra } \\
\text { Crespa } \\
1 \text { SESTERO DE TRIGO }\end{array}$ & & 1 SESTERO DE PAN \\
\hline & $\begin{array}{l}1 \text { PRADO encima del Brimal } \\
1 \text { MOSTELA DE YERBA }\end{array}$ & & \\
\hline LAS LLEVAN LOS DEL & $\begin{array}{l}1 \text { TIERRA en Lerumbre } \\
1 \text { SESTERO DE TRIGO }\end{array}$ & & 1 CUARTO DE TRIGO \\
\hline
\end{tabular}




\begin{tabular}{|c|c|c|c|}
\hline \multirow[t]{10}{*}{ CONCEJO DE COLIO } & $\begin{array}{l}1 \text { PRADO en la valleja de Oxido } \\
1 \text { CARGA DE YERBA }\end{array}$ & & \\
\hline & $\begin{array}{l}1 \text { TIERRA al nozal de La Llata } \\
1 / 2 \text { HEMINA DE TRIGO }\end{array}$ & 1 TIERRA al nozal del Bono & $1 / 2$ HEMINA DE PAN \\
\hline & $\begin{array}{l}1 \text { TIERRA en la Lintiguera } \\
1 / 2 \text { CELEMIN DE TRIGO }\end{array}$ & & 1 HEMINA DE TRIGO \\
\hline & $\begin{array}{l}1 \text { PRADO en la Serna } \\
1 / 2 \text { CARGA DE YERBA los } 1 / 2 \text { años }\end{array}$ & & 1 MOSTELA DE YERBA \\
\hline & $\begin{array}{l}1 \text { TIERRA en la Serna } \\
1 \text { CUARTO DE TRIGO }\end{array}$ & & $1 / 2$ FANEGA DE TRIGO \\
\hline & $\begin{array}{l}1 \text { TIERRA en la Serna al nozal de la } \\
\text { Llana } \\
1 / 2 \text { HEMINA DE TRIGO }\end{array}$ & & \\
\hline & $\begin{array}{l}1 \text { TIERRA al Hoyo } \\
1 \text { SESTREO DE TRIGO }\end{array}$ & & 1 HEMINA DE TRIGO \\
\hline & $\begin{array}{l}1 \text { TIERRA en so la Parte } \\
1 \text { CELEMIN DE TRIGO }\end{array}$ & & \\
\hline & $\begin{array}{l}1 \text { TIERRA en so la Parte } \\
1 \text { CELEMIN DE PAN }\end{array}$ & & 1 SESTERO DE PAN \\
\hline & $\begin{array}{l}1 \text { PRADO en Hullançes } \\
3 \text { CARGAS DE YERBA }\end{array}$ & & \\
\hline & $\begin{array}{l}1 \text { TIERRA en tras Carral } \\
1 / 2 \text { HEMINA DE TRIGO }\end{array}$ & 1 HEMINA DE TRIGO & \\
\hline $\begin{array}{l}\text { LAS LLEVAN LOS DEL } \\
\text { CONCEJO DE COLIO }\end{array}$ & $\begin{array}{l}1 \text { TIERRA en la Ribera } \\
1 \text { SESTERO DE TRIGO }\end{array}$ & & \\
\hline
\end{tabular}




\begin{tabular}{|c|c|c|}
\hline & $\begin{array}{l}1 \text { TIERRA en Gerico } \\
1 \text { SESTERO DE TRIGO }\end{array}$ & 1 SESTERO DE PAN \\
\hline & $\begin{array}{l}1 \text { TIERRA en las Cortynas } \\
1 \text { CUARTO DE TRIGO }\end{array}$ & \\
\hline & $\begin{array}{l}1 \text { TIERRA al Rio } \\
1 \text { HEMINA DE PAN }\end{array}$ & 1 HEMINA DE TRIGO \\
\hline & $\begin{array}{l}1 \text { TIERRA al Rodero } \\
1 \text { HEMINA DE TRIGO }\end{array}$ & \\
\hline & $\begin{array}{l}1 \text { TIERRA al Valle } \\
1 \text { SESTERO DE PAN }\end{array}$ & \\
\hline & $\begin{array}{l}1 \text { TIERRA en Hondon de la riega } \\
\text { del Valle } \\
1 \text { SESTERO DE PAN }\end{array}$ & NO FIGURA \\
\hline & $\begin{array}{l}1 \text { TIERRA en la Nabeda } \\
1 \text { FANEGA DE CENTENO }\end{array}$ & \\
\hline & $\begin{array}{l}1 \text { PRADO en Hondon del Esperon } \\
1 \text { CARGA DE YERBA }\end{array}$ & \\
\hline & $\begin{array}{l}1 \text { PRADO en Valdequexo } \\
2 \text { CARGAS DE YERBA }\end{array}$ & \\
\hline & $\begin{array}{l}1 \text { PRADO en Valdequexo } \\
1 \text { COLONO DE YERBA }\end{array}$ & 1 MOSTELA DE YERBA \\
\hline CONCEJO DE COLIO & $\begin{array}{l}1 \text { PRADO en Piedra Alvas } \\
1 \text { COLONO DE YERBA }\end{array}$ & 1 MOSTELA DE YERBA \\
\hline & $\begin{array}{l}1 \text { PRADO en Cerecedo } \\
4 \text { CARGAS DE YERBA }\end{array}$ & \\
\hline
\end{tabular}




\begin{tabular}{|c|c|c|c|}
\hline & $\begin{array}{l}1 \text { TIERRA en el vado de Yllana } \\
1 \text { SESTERO DE TRIGO }\end{array}$ & & 6 HEMINAS DE PAN \\
\hline & $\begin{array}{l}1 \text { TIERRA a Fuenquintyn } \\
1 \text { CUARTO DE PAN }\end{array}$ & & \\
\hline & $\begin{array}{l}1 \text { TIERRA en so villa } \\
1 \text { CUARTO DE PAN }\end{array}$ & 1 CUARTO DE TRIGO & 1 FANEGA DE TRIGO \\
\hline & $\begin{array}{l}1 \text { TIERRA en so villa a do dicen so } \\
\text { la Tapìa } \\
1 \text { CUARTO DE PAN }\end{array}$ & 1 HEMINA DE TRIGO & 1/2 HEMINA DE TRIGO \\
\hline & $\begin{array}{l}1 \text { TIERRA en syerra del Molino } \\
1 / 2 \text { EMINA DE TRIGO }\end{array}$ & & \\
\hline & $\begin{array}{l}1 \text { TIERRA so la Tapi } \\
3 \text { HEMINAS DE PAN }\end{array}$ & 3 HEMINAS DE TRIGO & \\
\hline & $\begin{array}{l}1 \text { HUERTO PEQUEÑO en Hondon } \\
\text { de villa } \\
\text { Unas almuezas de trigo }\end{array}$ & 1/2 CELEMIN DE TRIGO & 1 CELEMIN DE PAN \\
\hline & $\begin{array}{l}1 \text { TIERRA en la calle } \\
1 \text { puñado de pan }\end{array}$ & 1 puñado de TRIGO & 1 CELEMIN DE PAN \\
\hline & $\begin{array}{l}1 \text { PRADO en Estopino } \\
1 \text { COLONO DE YERBA }\end{array}$ & $1 / 2$ COLONO DE YERBA & 1 MOSTELA DE YERBA \\
\hline CONCEJO DE COLIO & $\begin{array}{l}1 \text { VIÑA en la Llata } \\
2 \text { AZUMBRES DE VINO }\end{array}$ & $\dot{¿} ?$ & 1 CESTO DE UVAS \\
\hline & $\begin{array}{l}1 \text { TIERRA al ponton de los prados } \\
\dot{¿} ?\end{array}$ & No se halla. & NO FIGURA \\
\hline
\end{tabular}




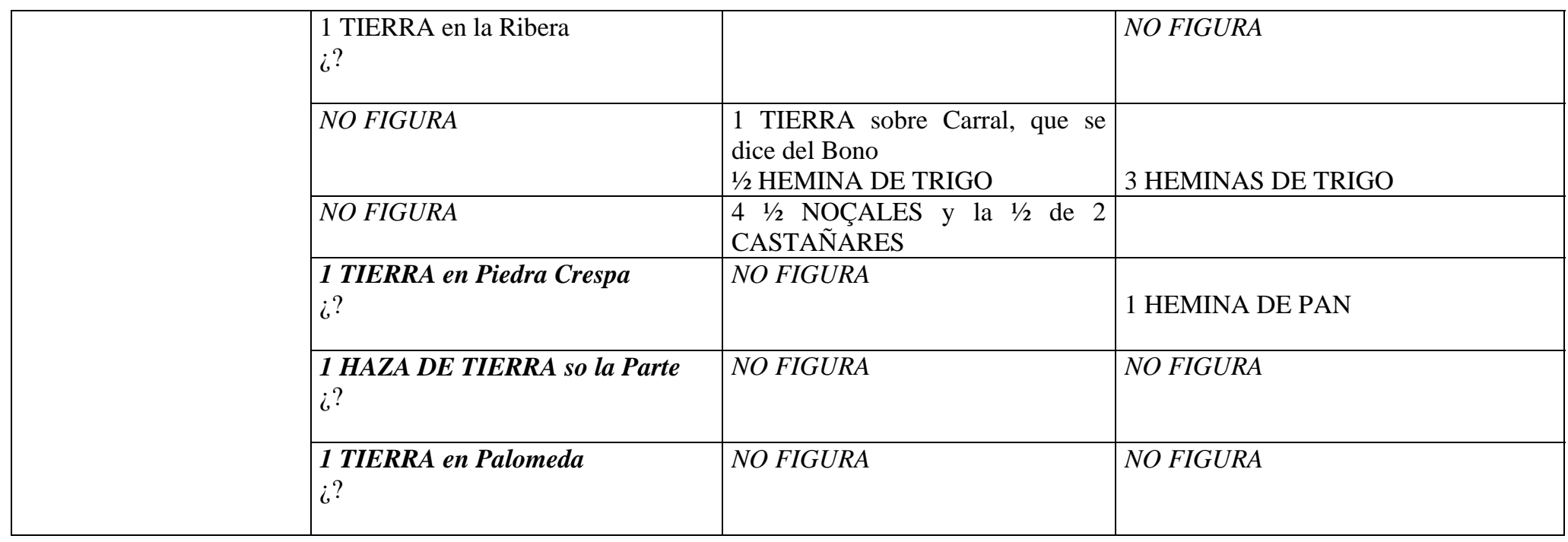

\section{CABAÑES}

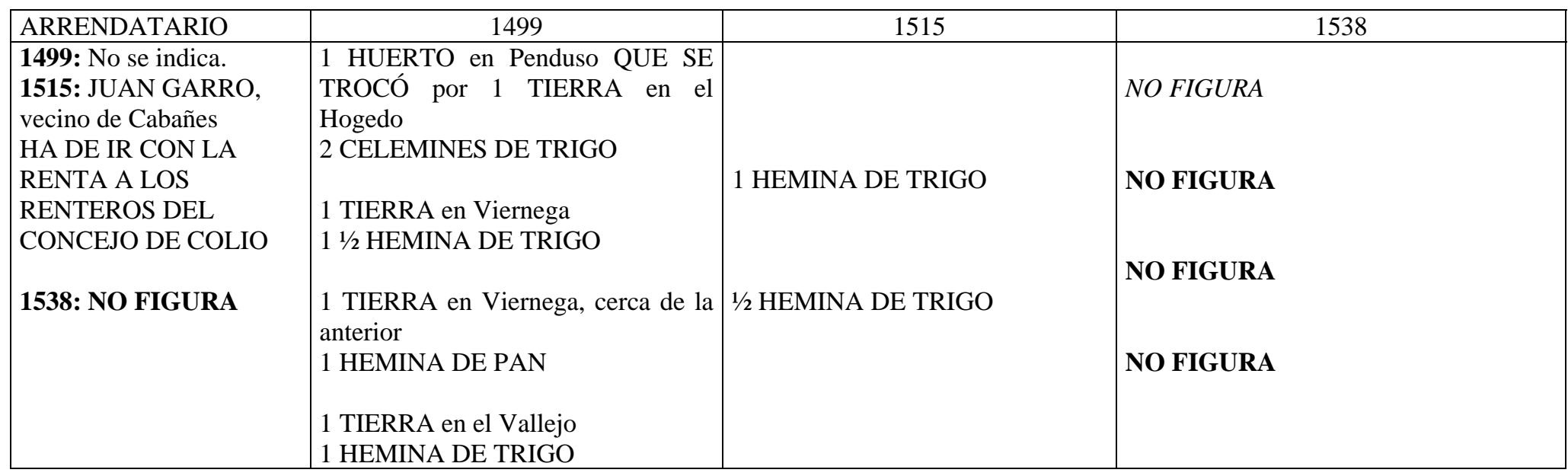


VIÑON. HEREDADES.

\begin{tabular}{|c|c|c|c|}
\hline BIENES & CAPACIDAD & OBSERVACIONES & \\
\hline $\begin{array}{l}\text { 1499: No se indica } \\
\text { 1515: Juan García, cura. } \\
\text { 1538: No se indica }\end{array}$ & $\begin{array}{l}1 \text { TIERRA so Santa María } \\
1 \text { SESTERO DE TRIGO }\end{array}$ & & 1 SESTERO DE PAN \\
\hline $\begin{array}{l}\text { 1499: No se indica } \\
\text { 1515: Fernando de Juan } \\
\text { de Redo y Garçia de } \\
\text { Vierguas, su cuñado. } \\
\text { 1538: No se indica }\end{array}$ & $\begin{array}{l}1 \text { VIÑA en Hospesendi, que es en } \\
\text { el cuerano de Cellena } \\
1 \text { CANTARA DE VINO }\end{array}$ & 4 CANTARAS DE VINO & \\
\hline $\begin{array}{l}\text { 1499: No se indica } \\
\text { 1515: PEDRO CORRAL } \\
\text { DE LLES } \\
\text { 1538: No se indica }\end{array}$ & $\begin{array}{l}1 \text { VIÑA en el término de Cellena } \\
2 \text { CÁNTARAS DE VINO }\end{array}$ & 1 CARGA DE UVAS & \\
\hline
\end{tabular}




\begin{tabular}{|c|c|c|c|c|c|c|c|c|c|c|c|c|c|}
\hline \multirow[t]{2}{*}{ Lugar } & \multicolumn{4}{|c|}{ Expl. Simples } & \multicolumn{9}{|c|}{ Expl. Complejas } \\
\hline & Tierra & Viña & Prado & Huerto & $\mathrm{T} / \mathrm{V}$ & $\mathrm{T} / \mathrm{P}$ & $\mathrm{T} / \mathrm{H}$ & $\mathrm{V} / \mathrm{P}$ & $\mathrm{V} / \mathrm{H}$ & $\mathrm{T} / \mathrm{V} / \mathrm{P}$ & $\mathrm{T} / \mathrm{V} / \mathrm{H}$ & $\mathrm{T} / \mathrm{P} / \mathrm{H}$ & $\mathrm{V} / \mathrm{P} / \mathrm{H}$ \\
\hline PENDES & & & & & & & & & & 1 & & & \\
\hline BEJES & & & & & & 1 & & & & & & & \\
\hline LEBEÑNA & 1 & 7 & 1 & & 7 & & & 2 & & & & & \\
\hline ENTERRÍAS & 8 & 1 & & & & 1 & & & & & & & \\
\hline DOBARGANES & & 1 & & & & & & & & & & & \\
\hline DOBRES & 1 & & & & & 1 & & & & & & & \\
\hline LA VEGA & 1 & & & & & & & & & & & & \\
\hline ARMAÑO & & & & & & & & & & 1 & & & \\
\hline TAMA & 1 & 1 & & & 1 & & & & & & & & \\
\hline ALIEZO & & & & & 4 & & & & & & & & \\
\hline OJEDO & & & & & & & & & & 2 & & & \\
\hline FRAMA & & & & & 3 & & & & & & & & \\
\hline CAMBARCO & 1 & & & & 2 & & & & & & & & \\
\hline CAHECHO & & & & & & & & & & 1 & & & \\
\hline TREVIÑO & & & & & & 1 & & & & & & & \\
\hline ENTERRÍA & & 1 & & & & 1 & & & & & & & \\
\hline BÁRCENA & 1 & & & & & 2 & & & & & & & \\
\hline LUARNA & & & 1 & & & & & & & & & & \\
\hline TANARRIO & 2 & 1 & 1 & & 1 & 8 & & & & 4 & & & \\
\hline BARÓ & 1 & 1 & & & 2 & 1 & & & & 1 & & & \\
\hline CAMALEÑO & 1 & & & & & & & & & & & & \\
\hline BODIA & & & 2 & & & 1 & & & & & & & \\
\hline CARAVAÑO & 1 & 3 & & & & & & & & & & & \\
\hline LA FRECHA & & & & & & 1 & & 1 & & 1 & & & \\
\hline BEARES & & & & & 1 & & & & & & & & \\
\hline ARGÜÉBANES & 3 & & & & 4 & 5 & & & & & & & \\
\hline CONGARNA & 1 & & & & 4 & 2 & & 1 & & 2 & & & \\
\hline FLORANES & & 3 & & & 1 & & & & & & & & \\
\hline TURIENO & & 3 & & 2 & 1 & & 1 & & 4 & 7 & 5 & & \\
\hline OTERO & & 1 & & & & & & 1 & & 1 & & & 1 \\
\hline MIESES & 2 & & & & 3 & & & & 1 & 2 & 5 & 1 & 1 \\
\hline \multirow[b]{2}{*}{ TOTAL } & 25 & 23 & 5 & 2 & 33 & 25 & 1 & 5 & 5 & 23 & 10 & 1 & 2 \\
\hline & \multicolumn{4}{|c|}{55} & \multicolumn{9}{|c|}{105} \\
\hline
\end{tabular}




\begin{tabular}{|c|c|c|c|c|}
\hline Lugar & Expl. Simples & $\%$ & Expl. Complejas & $\%$ \\
\hline PENDES & - & - & 1 & 100 \\
\hline BEJES & - & - & 1 & 100 \\
\hline LEBEÑA & 9 & 50 & 9 & 50 \\
\hline ENTERRÍAS & 9 & 90 & 1 & 10 \\
\hline DOBARGANES & 1 & 100 & & - \\
\hline DOBRES & 1 & 50 & 1 & 50 \\
\hline LA VEGA & 1 & 100 & & \\
\hline ARMAÑO & - & - & 1 & 100 \\
\hline TAMA & 2 & 66 & 1 & 33 \\
\hline ALIEZO & - & - & 4 & 100 \\
\hline OJEDO & - & - & 2 & 100 \\
\hline FRAMA & - & - & 3 & 100 \\
\hline CAMBARCO & 1 & 33 & 2 & 66 \\
\hline CAHECHO & - & - & 1 & 100 \\
\hline TREVIÑO & - & - & 1 & 100 \\
\hline ENTERRÍA & 1 & 50 & 1 & 50 \\
\hline BÁRCENA & 1 & 33 & 2 & 66 \\
\hline LUARNA & 1 & 100 & - & - \\
\hline TANARRIO & 4 & 24 & 13 & 76 \\
\hline BARÓ & 2 & 30 & 4 & 60 \\
\hline CAMALEÑO & 1 & 100 & - & - \\
\hline BODIA & 2 & 66 & 1 & 33 \\
\hline CARAVAÑO & 4 & 100 & - & - \\
\hline LA FRECHA & - & - & 3 & 100 \\
\hline BEARES & - & - & 1 & 100 \\
\hline ARGÜÉBANES & 3 & 25 & 9 & 75 \\
\hline CONGARNA & 1 & 10 & 9 & 90 \\
\hline FLORANES & 3 & 75 & 1 & 25 \\
\hline TURIENO & 5 & 22 & 18 & 78 \\
\hline OTERO & 1 & 33 & 2 & 66 \\
\hline MIESES & 2 & 14 & 13 & 86 \\
\hline TOTAL & 55 & 38 & 105 & 62 \\
\hline
\end{tabular}

